\title{
Online Social Network Data Placement over Clouds
}

\author{
Dissertation \\ zur Erlangung des mathematisch-naturwissenschaftlichen Doktorgrades \\ "Doctor rerum naturalium" \\ der Georg-August-Universität Göttingen
}

in the PhD Programme in Computer Science (PCS)

of the Georg-August University School of Science (GAUSS)

vorgelegt von

Lei Jiao

aus Shaanxi, China

Göttingen, 2014 


\section{Thesis Committee}

Prof. Dr. Xiaoming Fu

(Institute of Computer Science, University of Göttingen)

Prof. Jun Li, Ph.D.

(Department of Computer and Information Science,

University of Oregon, USA)

Prof. Dr. Dieter Hogrefe

(Institute of Computer Science, University of Göttingen)

\section{Members of the Examination Board}

Prof. Dr. Xiaoming Fu

(Institute of Computer Science, University of Göttingen)

Prof. Jun Li, Ph.D.

(Department of Computer and Information Science, University of Oregon, USA)

\section{Further Members of the Examination Board}

Prof. Dr. Dieter Hogrefe

(Institute of Computer Science, University of Göttingen)

Prof. Dr. Carsten Damm

(Institute of Computer Science, University of Göttingen)

Prof. Dr. Konrad Rieck

(Institute of Computer Science, University of Göttingen)

Prof. Dr. Ramin Yahyapour

(GWDG; Institute of Computer Science, University of Göttingen)

Date of the Oral Examination: 10 July 2014 


\section{Abstract}

Internet services today are often deployed and operated in data centers and clouds; users access and use the services by connecting to such data centers from their computing devices. For availability, fault tolerance, and proximities to users at diverse regions, a service provider often needs to run the service at multiple data centers or clouds that are distributed at different geographic locations, while aiming to achieve many system objectives, e.g., a service provider may want to reduce the money spent in using cloud resources, provide satisfactory service quality, data availability to users, limit the carbon footprint of the service, and so on. Inside a data center, a service provider is also concerned about some system objectives, e.g., running a service across servers may need to diminish the traffic that passes the oversubscribed core of the data center network.

A variety of system objectives can be addressed by carefully splitting and placing data at different clouds or servers. For instance, different clouds may charge different prices and emit different amounts of carbon for executing the same workload; they also have different proximities to users. Different servers inside a data center could reside at different positions in the data center network, where the traffic between servers at a common rack does not affect the network core but the traffic between servers at different racks may do. It is important for a service provider to make right decisions about where to place users' data over a group of clouds or servers, as data placement influences system objectives.

This thesis investigates the data placement problem for the Online Social Network (OSN) service, one of the most popular Internet services nowadays. Data placement for the OSN service has many challenges. First of all, users' data are interconnected. Ideally, the data of a user and the data of her friend should be co-located at the same cloud or server so that the user can access all the required data at a single site, saving any possible additional delay and traffic going across cloud or sever boundaries. Secondly, the master-slave replication complicates the data placement. A user may have a master replica that accepts both read and write operations and several slave replicas that only accept read operations; master and slave contribute differently to different system objectives and the best locations to place them can also be different. Thirdly, if multiple system objectives are considered, they are often intertwined, contradictory, and cannot be optimized simultaneously. Saving expense needs data to be placed at cheap clouds; reducing carbon prefers data to be placed at clouds with less carbon 
intensity; providing short latency requires data be placed close to users; data of friends also need to be co-located. All these requirements cannot be met at the same time and we desire a certain approach to seek trade-offs. On the other hand, in the scenario inside a data center, the topology of data center networks matters because, for different topologies, one often has different network traffic performance goals and thus different optimal data placements.

Our contribution is that we study three different settings of the OSN data placement problem by a combination of modeling, analysis, optimization, and extensive simulations, capturing real-word scenarios in different contexts while addressing all the aforementioned challenges. In the first problem, we optimize the service provider's monetary expense in using resources of geo-distributed clouds with guaranteed service quality and data availability, while ensuring that relevant users' data are always co-located. Our proposed approach is based on swapping the roles, master or slave, of a user's data replicas. In the second problem, we optimize multiple system objectives of different dimensions altogether when placing data across clouds by proposing a unified approach of decomposing the problem into two subproblems of placing masters and slaves respectively. We leverage the graph cuts technique to solve the master placement problem and use a greedy approach to place slaves. In the third problem, focused on the scenario inside a single data center, we encode different data center network topologies and performance goals into our data placement problem and solve it by borrowing our previous idea of swapping the roles of replicas and adapting it to reaching network performance goals while doing role-swaps. To validate our proposed approaches for each problem, we carry out extensive evaluations using real-world large-scale data traces. We demonstrate that, compared with state-of-the-art, de facto, and baseline methods, our approaches have significant advantages in saving the monetary expense, optimizing multiple objectives, and achieving various data center network performance goals, respectively. We also have discussions on complexity, optimality, scalability, design alternatives, etc. 


\section{Acknowledgements}

I have been fortunate to work with many people, without whose help this thesis would never have been possible. I owe tremendous thanks to them.

I give my deep appreciation to my advisor Prof. Dr. Xiaoming Fu. It was his constant guidance, support, and encouragement that spurred me to pursue research. His valuable assistance, suggestions, and feedback helped shape all my research work these years.

I am greatly indebted to Prof. Jun Li, Ph.D., who co-advised my doctoral study. He spent a lot of time revising, polishing, and improving almost every single paper of mine. Without his patience and efforts, this thesis would not have been what it is today.

My special gratitude goes to Dr. Wei Du. He had lots of insightful and fruitful discussions with me on the details of my work. I learnt and benefited hugely from our communications and collaboration.

I also thank Tianyin Xu and Dr. Yang Chen for their hands-on, useful advice.

My thanks in addition go to Prof. Dr. Dieter Hogrefe for being a member of my thesis committee; I also thank him, Prof. Dr. Carsten Damm, Prof. Dr. Konrad Rieck, and Prof. Dr. Ramin Yahyapour for serving as the examination board for me. Their comments made this thesis better.

I owe a great deal to my family. Their unconditional and endless love and support is always my motivation to go forward. To them I dedicate this thesis. 



\section{Contents}

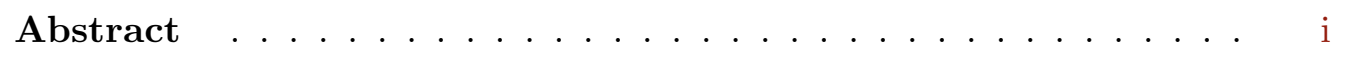

Acknowledgements ................. iii

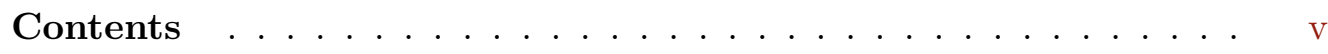

1 Introduction $\ldots \ldots \ldots \ldots \ldots \ldots$

1.1 Problem ........................... 1

1.2 Methodology . . . . . . . . . . . . . . . . 3

1.3 Contributions ........................ 5

1.3.1 Saving Expense while Ensuring Social Locality . . . . . . 5

1.3.2 Addressing Multiple Objectives via Graph Cuts . . . . 6

1.3.3 Achieving Data Center Network Performance Goals . . . 6

1.4 Deployment Considerations . . . . . . . . . . . . . 7

1.5 Thesis Organization . . . . . . . . . . . . . 7

2 Related Work . . . . . . . . . . . . . . . 11

2.1 Placing OSN across Clouds . . . . . . . . . . . . . . . . . . . . . . . . . . . . . 11

2.2 Placing OSN across Servers . . . . . . . . . . . . . . 12

2.3 Optimizing Cloud Services . . . . . . . . . . . . . . . 13

2.4 Graph Partitioning . . . . . . . . . . . . . . 15

3 OSN Data Placement across Clouds with Minimal Expense . 17

3.1 Introduction . . . . . . . . . . . . . . . 17

3.2 Models . . . . . . . . . . . . . . . . . . . . . . . . . 19

3.2 .1 System Settings _. . . . . . . . . . . . 19

3.2.2 Modeling the Storage and the Inter-Cloud Traffic Cost . 20

3.2.3 Modeling the Redistribution Cost . . . . . . . . . . . . 21

3.2.4 Approximating the Total Cost . . . . . . . . . . . 22

3.2.5 Modeling QoS and Data Availability ......... 24

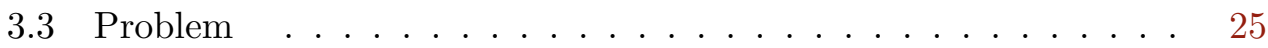

3.3.1 Problem Formulation . . . . . . . . . . . . 25

3.3.2 Contrast with Existing Problems . . . . . . . . . . . 27

3.3.3 NP-Hardness Proof . . . . . . . . . . . . . . . . . 27

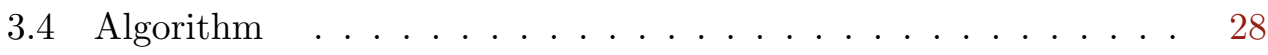


3.4 .1 Observations of Role-Swaps . . . . . . . . . . 28

3.4.2 Algorithm Based on Role-Swaps . . . . . . . . . . . . 29

3.5 Evaluations . . . . . . . . . . . . . . . . . 35

3.5.1 Data Preparation . . . . . . . . . . . 35

3.5.2 Experimental Settings . . . . . . . . . . . . . 37

3.5.3 Results on One-time Cost Reduction . . . . . . . . . 38

3.5.4 Results on Continuous Cost Reduction . . . . . . . . . 41

3.6 Discussions . . . . . . . . . . . . . . . . . . . . . . . 44

3.6.1 Algorithm Complexity . . . . . . . . . . . . 44

3.6 .2 Optimality Gap . . . . . . . . . . . . . . . . 44

3.6.3 Design Alternatives . . . . . . . . . . . . . . . . 45

3.6.4 Requirement Variation . . . . . . . . . . . 46

3.7 Summary . . . . . . . . . . . . . . . . . 47

4 OSN Data Placement across Clouds with Multiple Objectives 49

4.1 Introduction . . . . . . . . . . . . . . . . 49

4.2 Models . . . . . . . . . . . . . . . . . . 52

4.2 .1 System Settings _ . . . . . . . . . . . . 52

4.2.2 Modeling Carbon Footprint . . . . . . . . . . . 55

4.2 .3 Modeling Operation Distance _. . . . . . . . . . 55

4.2.4 Modeling Inter-Cloud Traffic . . . . . . . . . . . . . 56

4.2.5 Modeling Reconfiguration Cost . . . . . . . . . . . . 57

4.2 .6 Generalizing Models . . . . . . . . . . . . . . . 57

4.3 Problem . . . . . . . . . . . . . . . . . . 59

4.4 Algorithm . . . . . . . . . . . . . . . . 60

4.4.1 Decoupling Masters from Slaves . . . . . . . . . . 60

4.4.2 Solving Master Placement by Graph Cuts . . . . . . . . 61

4.4.3 Solving Slave Placement by a Greedy Approach . . . . . 62

4.5 Evaluations . . . . . . . . . . . . . . . . . 63

4.5.1 Data Preparation . . . . . . . . . . . . . 63

4.5.2 Experimental Settings _ . . . . . . . . . . . . 65

4.5.3 Optimization Results . . . . . . . . . . . . . 66

4.5.4 Algorithm Performance . . . . . . . . . . . . . . 69

4.6 Discussions . . . . . . . . . . . . . . . . . . . . . 72

4.6.1 Multi-Cloud Access Policies _. . . . . . . . . . 72

4.6.2 Optimality and Scalability _. . . . . . . . . . 74

4.7 Summary . . . . . . . . . . . . . . . . . . . . . 74

5 OSN Data Placement in Data Center for Network Performance 77

5.1 Introduction . . . . . . . . . . . . . . . . . 77

5.2 Models . . . . . . . . . . . . . . . . . 78 
5.2.1 Revisiting Social Locality . . . . . . . . . . . . . . 79

5.2.2 Encoding Network Performance Goals . . . . . . . . . . . 79

5.3 Problem . . . . . . . . . . . . . . . . . 80

5.4 Algorithm . . . . . . . . . . . . . . . . . . . . . 82

5.4.1 Achieving Performance Goals via Role-Swaps . . . . . . . 82

5.4.2 Traffic-Aware Partitioning Algorithm . . . . . . . . . . 83

5.5 Evaluations . . . . . . . . . . . . . . . . 85

5.5.1 Experimental Settings . . . . . . . . . . . . 85

5.5 .2 Evaluation Results _. . . . . . . . . . . 86

5.6 Discussions . . . . . . . . . . . . . . . . . . 88

5.7 Summary . . . . . . . . . . . . . . . . . 88

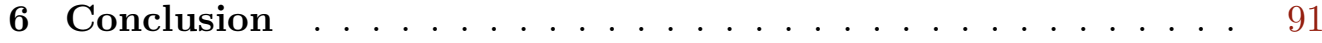

6.1 Comparative Summary _. . . . . . . . . . . . . . . . 91

6.2 Future Work . . . . . . . . . . . . . . . . . . 93

6.2.1 Data Placement vs. Request Distribution . . . . . . . . 93

6.2.2 Online Optimization over Time . . . . . . . . . . . 93

6.2.3 A Game Theoretic Perspective . . . . . . . . . . . . . . 94

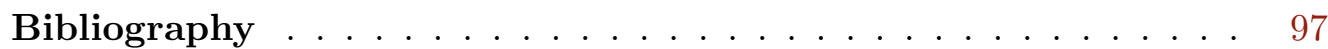





\section{Chapter 1}

\section{Introduction}

\subsection{Problem}

A large number of today's Internet services are deployed and operated in data centers $[17,23,47,89]$. Users access and use the services by connecting to data centers from their computers, mobile phones, or other devices. Data centers, the service infrastructure, provide resources like computation, storage, and network.

Cloud can provide "Infrastructure-as-a-Service" [34, 66, 71, 83] so that service providers do not have to build and maintain their own data centers; instead, they deploy their services in the cloud, which are built and operated by cloud providers, and pay for cloud resources that they use. A "cloud" here refers to a special data center that uses dedicated software to virtualize its resources and deliver them to customers. Running a service in the cloud has many advantages: cloud resources are ready to consume, letting service providers focus on their services rather than on building the service infrastructure which may not be their competence; cloud resources are "infinite", on demand, and can accommodate the surges of user requests, making it easy to scale the service; cloud resources are charged flexibly, "pay-as-you-go", and can save the expenses of service providers.

No matter operating a service in one's own data center or in the cloud, a service provider often needs its service to span multiple geographic areas for the purposes of availability, scalability, fault tolerance, and proximities to users at diverse regions [50, 80, 93, 94], with concerns on several different aspects. For instance, one may want to optimize the total monetary expense spent in using resources of multiple clouds [92, 98], including the cost of running virtual machines, storing data, and the cost for the traffic between clouds and between clouds and the users of the service, and so on. One may also want to provide good service quality, such as short access latency [61, 95], and satisfactory data availability $[24,49]$ to users. One may be even concerned about the carbon footprint of the service $[58,102]$, as carbon becomes an increasingly important issue nowadays. Depending on the specific scenarios, the concerns can be different.

A range of such concerns can be addressed by appropriately choosing at which data center or cloud to place which piece of data, given a group of candidate data centers or clouds that reside at different locations [13, 16, 40, 77]. For example, different clouds may charge different prices for consuming the same amount of resources, have different proximities to users, and emit different amounts 
of carbon for executing the same workload. Hence, data placement across the clouds can influence the performance and various system objectives of the service.

Inside a data center, choosing at which server to place which piece of data is also important. It is often not possible to host everything in a single server; splitting data across servers, however, needs to meet various performance goals $[32,72]$. Different servers may reside at different positions in the data center network. For example, the communication between some servers only passes one switch because the servers are at a common rack; the communication between some other servers may need to travel through more switches up to the core layer of the data center network topology as they are at different racks. Data placement in this case affects the paths that the inter-server communication travels along and thus further affects the usage of network resources.

This thesis specifically investigates the problem of placing Online Social Network (OSN) data both across multiple clouds or data centers, and across multiple servers inside a data center. OSN services are undoubtedly among the most popular Internet services nowadays. Facebook had 1.28 billion monthly active users as of March 31, 2014 [4]. Besides typical OSN services like Facebook and Twitter, an important observation is that "social" is gradually becoming a universal component of a large number of services, such as blogging, video sharing, and others, all what we may call the "socially aware" services.

There are some critical challenges for placing the data of OSN or the socially aware services over clouds and over servers inside a data center.

First of all, users of the OSN service are interconnected and the placements of their data are interdependent $[13,35,37,72]$. It is not that we choose the best location for a user's data, a cloud or a server, by considering the information of this user alone, but that when placing a user's data, we must also consider other users who access such data. The feature of OSN services is letting users form online friendships and communicate with one another, often by accessing the data of others. Each user is not independent and cannot be treated separately with regards to data placement. Ideally, for example, if the data of a user and the data of her friends are always co-located at the same cloud or sever, a user can access her friends' data without going to another cloud or server, and thus save any possible additional delay and traffic. This is unlike conventional webbrowsing services where users may not need to be jointly considered.

Secondly, the master-slave replication of users' data complicates the placement $[28,80]$. It is a common practice that a service may maintain multiple copies of a user's data, where one copy may be the master replica and the others are all slave replicas. When placing users' data, we must determine where to place each replica of each user. The difficulty is that different replicas serve different purposes and contribute differently to various system objectives. For example, a master replica accepts both read and write operations from either the 
user herself or her friends, while a slave replica accepts only read operations from users; besides, writes to a master need to be propagated to the corresponding slaves for consistency. The best location for a user's master may be different from that for a user's slave; where to place each of a user's salves is also an issue.

Thirdly, if multiple system objectives are considered altogether, they are often intertwined, contradictory, and cannot be accommodated simultaneously $[40,99]$. It is natural for a service provider to bear concerns from multiple dimensions, for example, monetary expense, QoS, carbon emission, as stated perviously. We cannot expect that a placement can address all such concerns to the best. To save money, we like cheap clouds; to provide good QoS, we prefer to place the data of a user at the cloud close to her; to make less carbon, we had better use those with less carbon intensity; besides, we should not forget that users' data are interdependent. When the clouds that are chosen to address each concern are different, as is often the case, we desire a certain approach to offer the capability of seeking trade-offs among multiple objectives.

In fact, the challenges are not limited to what have been stated here. In the wide-area multi-cloud scenario, we also need to consider how users access the data [82]. For instance, if a piece of required data is not present at the current cloud connected by a user, which other cloud with the required data this user should access determines where the read workload is executed and the corresponding carbon footprint is generated. In the local-area case inside a data center, the topology of the data center network matters if one wants to use data placement to dictate the network resource usage. In this thesis, we aim to address all such challenges.

\subsection{Methodology}

We attack the OSN data placement problem via studying the following three problem settings, corresponding to Chapter 3, Chapter 4, and Chapter 5, respectively. Chapters 3 and 4 investigate OSN data placement across clouds, and Chapter 5 investigates OSN data placement across servers inside a cloud. Fig. 1.1 is an overall picture of our work in this thesis. Users access their data in the OSN service. An OSN provider firstly needs to determine how to distribute users' data across multiple clouds, and then inside each cloud, it needs to determine how to distribute users' data across multiple servers.

The first problem setting aims to optimize the monetary expense that an OSN provider spends in using resources of multiple geo-distributed clouds, while providing satisfactory service quality and data availability to OSN users. In addition to modeling various costs of a multi-cloud OSN, the QoS requirement, and the data availability requirement, the core of this setting is ensuring for every user the social locality $[73,81]$, the access pattern that most activities of a 


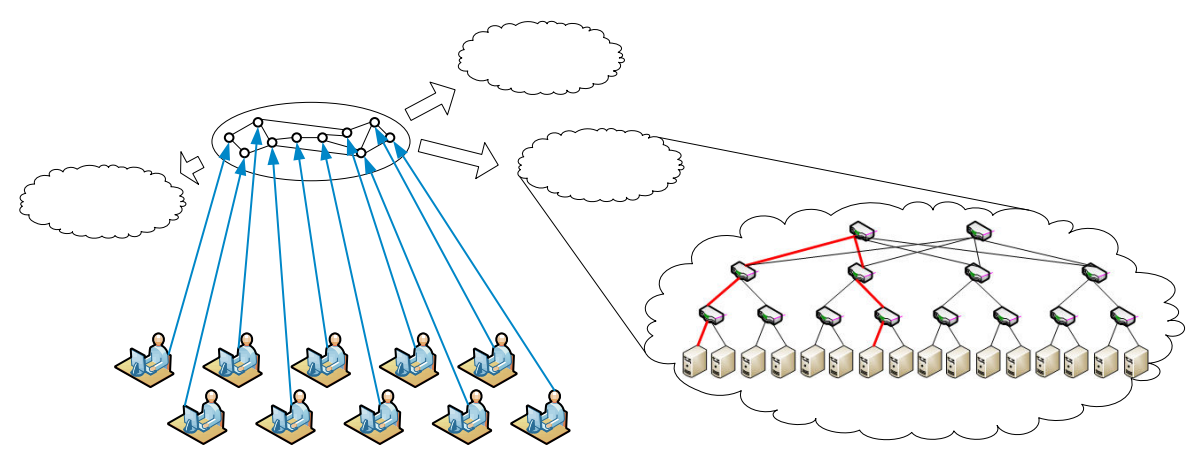

Figure 1.1: OSN data placement over clouds

user occur between herself and her neighbors [48, 90]. The data of a user and her friends must be co-located at a common cloud when optimizing data placement.

The second problem setting upgrades the attention to the multi-objective version of OSN data placement over geo-distributed clouds. In this setting, we are not limited to the monetary expense of an OSN provider; instead, we aim to optimize a number of system objectives of multiple dimensions simultaneously, including the carbon footprint, the service quality, the inter-cloud traffic, the reconfiguration cost, and so on. The core of this setting is capturing multiple objectives as functions of the data placement and other factors such as the master-slave replication [80] and the multi-cloud access policies [82], and figuring out whether these objectives can be treated, optimized by a unified approach.

The third problem setting focuses on OSN data placement across servers inside a single data center. We use social locality as a pre-condition that must be satisfied, which also comes with the traffic overhead of maintaining replica consistency across servers. Inside a data center, servers are connected by dedicated network topologies, e.g., tree [11, 87], Clos topology [14, 15]. The core of this setting is encoding the differing network or traffic performance goals of a variety of modern data center network topologies into our data placement problem so that the optimal data placement can lead to the optimal network performance.

The focus of this thesis is on making intelligent decisions, e.g., where to place which data. To this end, we take the following approach to carry out our research for each problem setting: "Models" $\rightarrow$ "Problem" $\rightarrow$ "Algorithm" $\rightarrow$ "Evaluations" $\rightarrow$ "Discussions". Firstly, we mathematically model the specific problem setting under reasonable assumptions and conditions. Afterwards, based on the models, we formulate the data placement problem in the language of optimization: we have either a single objective or multiple objectives, with or without constraints; we also have decision variables representing the locations of each piece of data. Further, we analyze the optimization problem and propose algorithms to find good solutions. Then, we use real-world large-scale data traces as inputs to extensively evaluate our algorithms. The outputs are 
compared with those produced by other state-of-the-art, de facto, or baseline approaches. We interpret and explain the evaluation results. Finally, we discuss the various aspects such as complexity, optimality, scalability, design alternatives, and so on. We either do additional evaluations to assist our discussions or conduct discussions only based on our models.

\subsection{Contributions}

We study the three different problem settings as stated previously, capturing real-world scenarios in different contexts, by a combination of modeling, analysis, optimization, and simulation.

\subsubsection{Saving Expense while Ensuring Social Locality}

In the first problem setting, we model the cost of an OSN as the objective, and model the QoS and the data availability requirements as the constraints of the data placement optimization problem. Our cost model identifies different types of costs associated with a multi-cloud OSN, including the storage cost and the inter-cloud traffic cost incurred by storing and maintaining users' data in the clouds, as well as the redistribution cost incurred by our optimization mechanism itself. All kinds of costs ensure the social locality [73, 81] for every user as a premise. Translated into the master-slave paradigm that we consider, it means that a user's every neighbor must have either a master replica or a slave replica at the cloud that hosts the user's own master replica. Our QoS model links the QoS of the OSN service with the locations of all users' master replicas over clouds. Our data availability model relates with the minimum number of replicas of each user. We prove the NP-hardness of the optimization problem.

Our core contribution is an algorithm named cosplay that is based on our observations that swapping the roles (i.e., master or slave) of a user's data replicas on different clouds can not only lead to possible cost reduction, but also serve as an elegant approach to ensuring QoS and maintaining data availability. We carry out extensive experiments by distributing a real-world geo-social Twitter dataset of 321,505 users with 3,437,409 social relations over 10 clouds all across the US in a variety of settings. Our results demonstrate that, while always ensuring the QoS and the data availability as required, cosplay can reduce much more one-time cost than the state of the arts, and it can also significantly reduce the accumulative cost when continuously evaluated over 48 months, with OSN dynamics comparable to real-world cases. We analyze that cosplay has quite a moderate complexity, and show that cosplay tends to produce data placements within a reasonably good optimality gap towards the global optimum. We also discuss other possible design alternatives and extended use cases. 


\subsubsection{Addressing Multiple Objectives via Graph Cuts}

In the second problem setting, we allow every user having one master replica and a fixed number of slave replicas, based on which we model various system objectives including the carbon footprint, the service quality, the inter-cloud traffic, as well as the reconfiguration cost incurred by changing one data placement to another, considering the multi-cloud access policies. The big change compared with the first problem setting is that we give up ensuring social locality for every user. A possible consequence of this change is that we find all the models of system objectives composed of one or both of the two parts: a unary term that only depends on the locations of a single user's replicas and a pairwise term that depends on the locations of the replicas of a pair of users. Besides, our models can be generalized to cover a wide range of other system objectives.

Our core contribution here is a unified approach to optimize the multiple objectives. We propose to decompose our original data placement problem into two simpler subproblems and solve them alternately in multiple rounds: in one problem, given the locations of all slaves, we identify the optimal locations of all masters by iteratively invoking the graph cuts technique $[25,26,56]$; in the other subproblem, we place all slaves given the locations of all masters, where we find that the optimal locations of each user's slaves are independent and a greedy method that takes account of all objectives can be sufficient. We conduct evaluations using a real-world dataset of 107,734 users interacting over 2,744,006 social relations, and place these users' data over 10 clouds all across the US. We demonstrate results that are significantly better than standard and de facto methods in all objectives, and also show that our approach is capable of exploring trade-offs among objectives, converges fast, and scales to a huge user base. While proposing graph cuts to address master replicas placement, we find that different initial placements of all replicas and different methods of placing slave replicas can influence the optimization results to different extents, shedding light on how to better control our algorithm to achieve desired optimizations.

\subsubsection{Achieving Data Center Network Performance Goals}

In the third problem setting, we consider a diversity of modern data center network topologies inside the data center, identify the different network or traffic performance goals, and encode these goals into our data placement optimization problem. While a general network performance goal would be minimizing the sum of the amount of traffic passing every router, in the conventional threelayer tree topology with heavy oversubscription minimizing the amount of traffic passing the core-layer routers seems more important. Here in this setting we still ensure social locality, which comes with the storage overhead of slave replicas and the network overhead of the traffic of maintaining replica consistency across 
servers. We aim to align such traffic with various network performance goals by carefully selecting servers to place each user's master and slave replicas, while guaranteeing that the storage overhead does not increase.

Our contribution here is borrowing our previous idea of swapping the roles of data replicas and adapting it to achieving network performance goals during role-swaps. Through evaluations with a large-scale, real-world Twitter trace, we show that in a variety of data center network topologies with a varying number of servers, compared with state-of-the-art algorithms, our algorithm significantly reduces traffic, achieving various network performance goals without deteriorating the load balance among servers and causing extra replication overhead.

\subsection{Deployment Considerations}

We outline a possible architecture for implementing and incorporating our algorithmic contributions into real-world systems. We need to implement three components: the Information Collector (IC), the Problem Solver (PS), and the Decision Executor (DE). The IC is responsible for collecting the social graph, the amount of interactions among users, the network latencies between clouds and between users and clouds, the carbon intensities of clouds, and all other information that is needed as inputs to our algorithms. The PS, where our algorithms are actually implemented, is responsible for running the algorithms to solve the corresponding optimization problem and make intelligent decisions about data placement. The DE is responsible for collecting the outputs of our algorithms and invoking other related system components and services to implement the decisions by moving data from their current locations to the new locations. The division of the three components here is in a logical sense; physically, they can be implemented as a single software component or multiple software components running at one server or multiple servers.

In a multi-cloud scenario such as Fig. 1.2, each cloud runs an IC and a DE, and one of the clouds runs the PS. The IC at each cloud reports the inputs to the PS, and the PS makes decisions and communicates with the DE at each cloud to coordinate the data movements across clouds. DEs may also need to communicate with one another to send and receive data. In this figure, each cloud hosts some data of users; for simplicity, we do not draw them. Note that, by regarding each cloud in this figure as a server, this architecture may also be used for data placement inside a data center.

\subsection{Thesis Organization}

This thesis contains part of the content of the following published papers. 


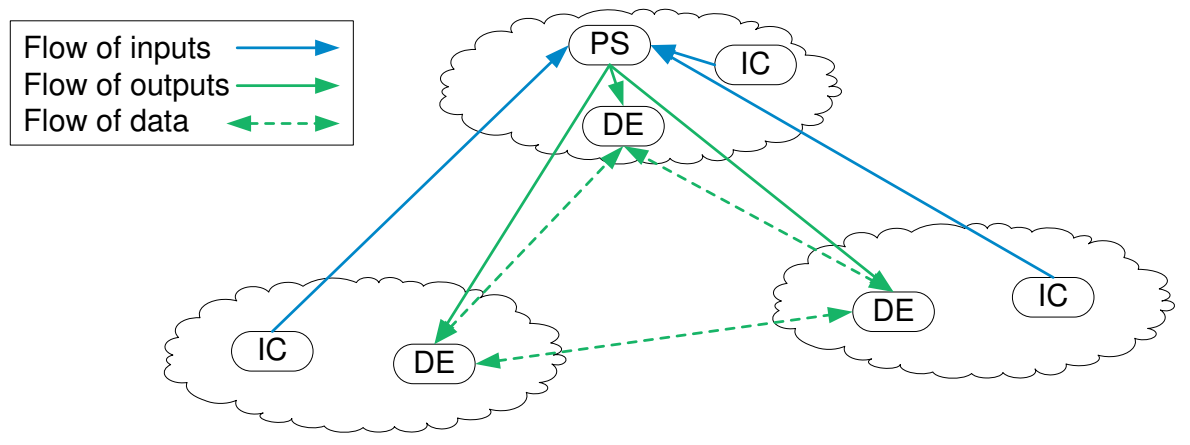

Figure 1.2: A simple architecture for deployment

- Lei Jiao, Jun Li, Tianyin Xu, Xiaoming Fu, "Cost Optimization for Online Social Networks on Geo-Distributed Clouds", in proceedings of the 20th IEEE International Conference on Network Protocols (ICNP), Austin, Texas, USA, October 2012

- Lei Jiao, Jun Li, Wei Du, Xiaoming Fu, "Multi-Objective Data Placement for Multi-Cloud Socially Aware Services", in proceedings of the 33rd IEEE International Conference on Computer Communications (INFOCOM), Toronto, Canada, April 2014

- Lei Jiao, Jun Li, Xiaoming Fu, "Optimizing Data Center Traffic of Online Social Networks", in proceedings of the 19th IEEE International Workshop on Local and Metropolitan Area Networks (LANMAN), Best Paper Award, Brussels, Belgium, April 2013

This thesis is structured as follows. Chapter 1 provides an overview of this thesis: introducing the data placement problem and the challenges for OSN data placement, stating our research methodology and contributions, and outlining our deployment considerations and the structure of this thesis. Chapter 2 presents the related work in multiple categories, and highlights how our work in this thesis differs from them and bridges the gap. Chapter 3, based on our first publication as mentioned above, describes our work on minimizing the monetary expense that an OSN provider spends in using resources of geo-distributed clouds while providing satisfactory QoS and data availability to OSN users. Chapter 4, based our second publication as mentioned above, describes our work on optimizing the OSN data placement over multiple clouds with multiple objectives of diverse dimensions by a unified decomposition approach based on graph cuts and a comprehensive greedy method. Chapter 5, based on our third publication as mentioned above, describes our work on placing data across servers inside a single data center, aiming to achieve different network performance goals for different data center network topologies. Chapter 6 summarizes this thesis by 
comparing our studies in Chapters 3, 4 and 5 with one another, and finally shares some of our thoughts on the future work. 



\section{Chapter 2}

\section{Related Work}

We discuss existing work in four categories, and for each category, we summarize how the work fails to address the challenges that are faced by placing OSN over clouds, and highlight how our work in this thesis bridges the gap. The first two categories are on placing and optimizing the data of OSN and social media across clouds and across servers inside a cloud, which may be the work most related to ours. The third category is about socially oblivious cloud services, demonstrating what can be some important performance metrics of cloud services and how they can be optimized. The last category, from a graph theory perspective, introduces the study of graph partitioning and repartitioning problems that seem similar to our problems of splitting and replicating OSN.

\subsection{Placing OSN across Clouds}

The multi-cloud or multi-data-center platform is promising for deploying and operating OSN and socially aware services. A branch of existing work investigates the challenges and opportunities towards this direction.

Liu et al. [60] focused on the inter-data-center communication of the OSN service. Maintaining a replica of a remote user's data at a local data center reduced the inter-data-center read operations as local users could access such data without going to remote data centers; however, this replica at the local data center needed to be updated for consistency with remote replicas and thus incurred the inter-data-center update operations. The authors proposed to replicate across data centers only the data of the users selected by jointly considering the read and the update rates in order to ensure that a replica could always reduce the total inter-data-center communication.

Wittie et al. [91] claimed that Facebook had slow response to users outside US and Internet bandwidth was wasted when users worldwide requested the same content. The authors found that the slow response was caused by the multiple round trips of Facebook communication protocols as well as the high network latency between users and Facebook US data centers; they also observed that most communications were among users within the same geographic region. The authors proposed to use local servers as TCP proxies and caching servers to improve service responsiveness and efficiency, focusing on the interplay between user behavior, OSN mechanisms, and network characteristics. 
Wu et al. [92] advocated using geo-distributed clouds for scaling the social media streaming service. However, the challenges remained for storing and migrating media data dynamically in the clouds for timely response and moderate expense. To address such challenges, the authors proposed a set of algorithms that were able to do online data migration and request distribution over consecutive time periods based on Lyapunov optimization techniques. They predicted the user demands by exploiting the social influence among users; leveraging the predicted information, their algorithms could also adjust the online optimization result towards the offline optimum.

Wang et al. [86] targeted social applications which often had a workflow of "collection" $\rightarrow$ "processing" $\rightarrow$ "distribution". The authors proposed local processing, which collected and processed user-generated content at local cloud$\mathrm{s}$, and global distribution, which delivered processed content to users via geodistributed clouds, as a new principle to deploy social applications across clouds, and designed protocols to connect these two components. They modeled and solved optimization problems to determine computation allocation and content replication across clouds, and built prototypes in real-world clouds to verify the advantages of their design.

The work in this category focuses on the performance of OSN services [60, 91] and social applications [86], and the monetary expense of provisioning and scaling social media in the clouds [92]. Our work in this thesis investigates the monetary expense of the OSN service with its QoS and data availability requirements, as well as the many other facets of the OSN performance over clouds. To the best of our knowledge, we are the first to include the carbon footprint of OSN services into consideration, with a complex trade-off among a large variety of related factors such as QoS and inter-cloud traffic. In addition to the generality of our models that can capture a diversity of performance metrics and the uniqueness of our proposed algorithmic approach, we investigate this complicated joint optimization problem in the context of master-slave replication while accommodating different multi-cloud access policies.

\section{$2.2 \quad$ Placing OSN across Servers}

At a single site, how to partition and replicate the data of OSN and socially aware services across servers remains another important problem. A body of existing literature tackles this scenario.

OSN services often adopt distributed hashing to partition the data across servers $[7,57]$, which can lead to poor performance such as unpredictable response time due to the inter-server multi-get operations, and the response time is determined by the server with the highest latency. To address this problem, recent work proposed to eliminate the inter-server multi-get operations by main- 
taining social locality, i.e., replicating friends across servers $[72,81]$ so that all friends of a user could be accessed at a single server. SPAR [72] minimized the total number of slave replicas while maintaining social locality for every user and balancing the number of master replicas in each partition. S-CLONE [81] maximized the number of users whose social locality could be maintained given a fixed number of replicas for each user. Another approach [30] to tackle the same problem explored self-similarities, a feature that was found in OSN interactions and did not exist in OSN social relations. Self-similarity was known as a driving force to minimize the dissipation of cost/energy in dynamic process. The authors argued that placing users in the same self-similar subtree at the same server minimized the inter-server communication.

Carrasco et al. [27] noticed that an OSN user's queries often only cared about the most recent messages of friends, and thus dividing messages according to time stamps and placing those within a particular time range at a particular server had far less storage overhead than partitioning messages only based on OSN friendships. Partitioning along the time dimension could also serve as an approach to optimize OSN performance.

Cheng et al. [31] considered partitioning social media content across servers. The authors found that when doing such partitioning, not only the social relations should be considered, one also needed to consider the user access patterns to each media file otherwise the viewing workload at each server could be skewed. The authors formulated an optimization problem and solved it to preserve social relations and to balance the workload among servers.

The work in this category is mainly on OSN and social media placement optimization across servers at a single site. All such existing work cares more often the server performance, and very little has been done on the network performance. The root cause is that they essentially target a server cluster environment, instead of a data center environment where the network performance also needs considerable attention. Our work in this thesis identifies the network performance goals for different data center networks, captures and encodes them into our optimization problem. We propose a unified algorithm to place OSN data across servers to optimize a diversity of such goals while maintaining social locality. Our work is done in a way that we optimize network performance without hurting server performance, i.e., without affecting the existing load balance among servers and increasing the total replication overhead.

\subsection{Optimizing Cloud Services}

There exists rich research work on optimizing cloud services. Besides conventional performance metrics such as service latency, energy and carbon increasingly becomes an important concern of optimization in recent years. 
Qureshi et al. [74] might be the first to propose to exploit the difference of electricity prices for clouds at different geo-locations. Electricity prices exhibited both temporal and geographic variations due to various reasons such as regional demand differences, transmission inefficiencies, and diversities of generation sources. Distributing requests to adjust the workload of each cloud could thus lead to significant monetary savings for the total electric bills of all the clouds. The authors also used bandwidth and performance as constraints.

Rao et al. [76] further observed that data centers could consume electricity from multiple markets: some US regions had wholesale electricity markets where electricity prices might vary on an hourly or a 15-minute basis while the prices in other regions without wholesale markets could remain unchanged for a longer time period. The authors proposed to leverage both the market-based and the location-based price differences to minimize the total electric bill while guaranteeing the service delay captured by a queueing model.

Le et al. [58] studied the multi-data-center energy expense problem in a different setting. The authors argued that the brown energy, i.e., which was produced by coal, should be capped and the green energy, i.e., which was produced by wind, water, etc., should be explored. Their work proposed a model framework to capture the energy cost of services in the presence of brown energy caps, data centers that could use green energy, and multiple electricity prices and carbon markets. The authors minimized the energy cost by distributing requests among data centers properly while abiding by service level agreements.

$\mathrm{Xu}$ et al. [95] jointly optimized the electricity cost and the bandwidth cost of geo-distributed data centers. Electricity cost could be optimized via distributing requests to data centers as stated previously. There was also room for bandwidth cost optimization. Nowadays a data center often connected to multiple ISPs simultaneously and a request, once processed at a data center, needed to be routed back to the user via one of the available ISP links which often had different prices. To exploit both price differences of electricity and bandwidth, the authors modeled an optimization problem and solved it by a distributed algorithm.

Gao et al. [40], to the best of our knowledge, did the only work so far of optimizing multiple dimensions of system objectives of distributed data centers or clouds. The authors optimized carbon footprint, electricity cost, and access latency through proper request distribution and content placement across data centers. They proposed an optimization framework that allowed data center operators to navigate the trade-offs among the three dimensions; they also studied using their framework to do carbon-aware data center upgrades. A heuristic was also available to achieve approximate solutions at a faster speed.

Besides electricity and carbon, a substantial body of literatures studies cloud resource pricing [78] and allocation [54], as well as a range of other related issues in the cloud scenario. We are not going into further details here. 
This category of work targets conventional and socially oblivious services. Except [40], they often assume full data replication across data centers; even [40] still cannot serve our purpose. The existing work does not address (1) social relations and user interactions, $(2)$ writes to contents and the maintenance of replica consistency, (3) inter-cloud operations that contribute to QoS and intercloud traffic, and (4) the master-slave replication that is widely used in reality, and thus falls short in the problem space in the first place. In contrast, our work in this thesis captures all such particular features in the context of socially aware services and provides trade-offs among a wide range of system performance metrics via our generalized model framework and a unified algorithmic approach.

\subsection{Graph Partitioning}

In the last part of this related work section, we briefly introduce the existing study of graph partitioning and repartitioning problems. Conventionally, such problems are studied from a graph theoretic and algorithmic perspective.

Graph partitioning aims to divide a weighted graph into a specified number of partitions in order to minimize either the weights of edges that straddle partitions or the inter-partition communication while balancing the weights of vertices in each partition [12]; graph repartitioning additionally considers the existing partitioning, and pursues the same objective as graph partitioning while also minimizing the migration costs [79]. State-of-the-art algorithms and solutions to such problems include METIS [53] and Scotch [70]. We take METIS here as an example. METIS is a multi-level partitioning algorithm that is composed of three phases: the coarsening phase, the partitioning phase, and the uncoarsening phase. In the coarsening phase, vertices are merged iteratively dictated by some rules and thus the size of the original graph becomes smaller and smaller. In the partitioning phase, the smallest graph is partitioned. In the uncoarsening phase, the partitioned graph is projected back to finer graphs iteratively and the partitioning is also refined following some algorithms until one gets the finest, original graph. There are many of such merging rules and refining algorithms that one can consider for a specific instance of a graph partitioning problem.

The problems we study in this thesis have some fundamental differences from the classic graph partitioning and repartitioning problems. Classic problems handle weighed graphs and have no notion of social locality, QoS, data availability, carbon, data center network topologies, etc., which makes their algorithms inapplicable to our cases, e.g., minimizing the total inter-server communication does not necessarily minimize the traffic traveling via the core-layer switches or the total traffic perceived by every switch, nor the carbon footprint and the access latency. To solve problems that capture such concerns, in this thesis, we propose novel algorithms based on swapping the roles of data replicas and graph cuts. 



\section{Chapter 3}

\section{OSN Data Placement across Clouds with Minimal Expense}

\subsection{Introduction}

Internet services today are experiencing two remarkable changes. One is the unprecedented popularity of Online Social Networks (OSNs), where users build social relationships, and create and share contents with one another. The other is the rise of clouds. Often spanning multiple geographic locations, clouds provide an important platform for deploying distributed online services. Interestingly, these two changes tend to be combined. While OSN services often have a very large user base and need to scale to meet demands of users worldwide, geo-distributed clouds that provide Infrastructure-as-a-Service can match this need seamlessly, further with tremendous resource and cost efficiency advantages: infinite on-demand cloud resources can accommodate the surges of user requests; flexible pay-as-you-go charging schemes can save the investments of service providers; and cloud infrastructures also free service providers from building and operating ones' own data centers. Indeed, a number of OSN services are increasingly deployed on clouds, e.g., Sonico, CozyCot, and Lifeplat [2].

Migrating OSN services towards geographically distributed clouds must reconcile the needs from several different aspects. First, OSN providers want to optimize the monetary cost spent in using cloud resources. For instance, they may wish to minimize the storage cost when replicating users' data at more than one cloud, or minimize the inter-cloud communication cost when users at one cloud have to request the data of others that are hosted at a different cloud. Moreover, OSN providers hope to provide OSN users with satisfactory quality of service (QoS). To this end, they may want a user's data and those of her friends to be accessible from the cloud closest to the user, for example. Last but not least, OSN providers may also be concerned of data availability, e.g., ensuring the number of users' data replicas to be no fewer than a specified threshold across clouds. Addressing all such needs of cost, QoS, and data availability is further complicated by the fact that an OSN continuously experiences dynamics, e.g., new users join, old users leave, and the social relations also vary.

Existing work on OSN service provisioning either pursues the least cost at 
a single site without the QoS concern as in the geo-distribution case [73, 81], or aims at the least inter-data-center traffic in the case of multiple data centers without considering other dimensions of the service [60], e.g., data availability. More importantly, the models in all such work do not capture the monetary cost of resource usage and thus cannot fit the cloud scenario. There are some work on cloud-based social video [86, 92], focusing on leveraging online social relationships to improve video distribution, however still leaving a gap towards the OSN service; most optimization research on multi-cloud and multi-data-center services are not for OSN $[13,54,78,95]$. They do not capture the OSN features such as social relationships and user interactions, neither can their models be applicable to OSN services.

In this chapter, we therefore study the problem of optimizing the monetary cost of the dynamic, multi-cloud-based OSN, while ensuring its QoS and data availability as required.

We first model the cost, the QoS, and the data availability of the OSN service upon clouds. Our cost model identifies different types of costs associated with multi-cloud OSN while capturing social locality [73, 81], an important feature of the OSN service that most activities of a user occur between herself and her neighbors. Guided by existing research on OSN growth and our analysis of real-world OSN dynamics, our model approximates the total cost of OSN over consecutive time periods when the OSN is large in user population but moderate in growth, enabling us to achieve the optimization of the total cost by independently optimizing the cost of each period. Our QoS model links the QoS with OSN users' data locations among clouds. For every user, all clouds available are sorted in terms of a certain quality metric (e.g., access latency); therefore every user can have the most preferred cloud, the second most preferred cloud, and so on. The QoS of the OSN service is better if more users have their data hosted on clouds of a higher preference. Our data availability model relates with the minimum number of replicas maintained by each OSN user.

We then base on these models to formulate the cost optimization problem which considers QoS and data availability requirements. We prove the NPhardness of our problem. We propose an algorithm named cosplay based on our observations that swapping the roles (i.e., master or slave) of a user's data replicas on different clouds can not only lead to possible cost reduction, but also serve as an elegant approach to ensuring QoS and maintaining data availability. Compared with existing approaches, cosplay reduces cost significantly and finds a substantially good solution of the cost optimization problem, while guaranteeing all requirements are satisfied. Furthermore, not only can cosplay reduce the onetime cost for a cloud-based OSN service, by estimating the heavy-tailed OSN activities [21, 84] during runtime, it can also solve a series of instances of the cost optimization problem and thus minimize the aggregated cost over time. 
We further carry out extensive experiments. We distribute a real-world geosocial Twitter dataset of 321,505 users with 3,437,409 social relations over 10 clouds all across the US in a variety of settings. Compared with existing alternatives, including some straightforward methods such as the greedy placement (the common practice of many online services [80, 82]), the random placement (the de facto standard of data placement in distributed DBMS such as MySQL and Cassandra [57]), and some state-of-the-art algorithms such as SPAR [73] and METIS [53], cosplay produces better data placements. While meeting all requirements, it can reduce the one-time cost by up to about $70 \%$. Further, over 48 consecutive months with OSN dynamics comparable to real-world cases, compared with the greedy placement, continuously applying cosplay can reduce the accumulative cost by more than $40 \%$. Our evaluations also demonstrate quantitatively that the trade-off among cost, QoS, and data availability is complex, and an OSN provider may have to try cosplay around all the three dimensions. For instance, according to our results, the benefits of cost reduction decline when the requirement for data availability is higher, whereas the QoS requirement does not always influence the amount of cost that can be saved.

The remainder of this chapter is structured as follows. Section 3.2 describes our models of the cost, QoS, and data availability of the OSN service over multiple clouds. Section 3.3 formulates the cost optimization problem. Section 3.4 elaborates our cosplay algorithm, as well as our considerations and insights behind. Section 3.5 demonstrates and interprets our evaluations. Section 3.6 discusses some related issues such as complexity and optimality. Section 3.7 concludes.

\subsection{Models}

Targeting the OSN service over multiple clouds, we begin with identifying the types of costs related to cloud resource utilization: the storage cost for storing users' data, the inter-cloud traffic cost for synchronizing data replicas across clouds, the redistribution cost incurred by the cost optimization mechanism itself, and some underlying maintenance cost for accommodating OSN dynamics. We discuss and approximate the total cost of the multi-cloud OSN over time. Afterwards, we propose a vector model to capture the QoS of the OSN service, show the features of this model, and demonstrate its usage. Finally, we model the OSN data availability requirement by linking it with the minimum number of each user's data replicas.

\subsubsection{System Settings}

Clouds and OSN users are all geographically distributed. Without loss of generality, we consider the single-master-multi-slave paradigm [20, 80]: each user has 
only one master replica and several slave replicas of her data, where each replica is hosted at a different cloud. When signing in to the OSN service, a user always connects to her master cloud, i.e., the cloud that hosts her master replica, and every read or write operation conducted by a user goes to her master cloud first.

We assume the placement of OSN users' replicas follows the social locality scheme $[73,81]$. Observing that most activities of an OSN user happen between the user and her neighbors (e.g., friends on Facebook or followees on Twitter), this scheme requires that a user's master cloud host a replica (either the master or a slave) of every neighbor of the user. This way, every user can read the data of her friends and her own from a single cloud, and the inter-cloud traffic only involves the write traffic for maintaining the consistency among a user's replicas at different clouds. Social locality has multi-fold advantages: given that there are often many more reads than writes in an OSN service [22], it can thus save a large proportion of the inter-cloud traffic; this scheme also incurs a much lower storage consumption than full replication in that the full replication requires every cloud to maintain a data replica for every user. Note that for a user with one master and $r$ slaves, a write on this user's data always incurs $r$ corresponding inter-cloud writes to maintain consistency. We consider eventual consistency in our work, and assume issues such as write conflicts are tackled by existing techniques.

\subsubsection{Modeling the Storage and the Inter-Cloud Traffic Cost}

OSN is commonly abstracted as a social graph, where each vertex represents a user and each edge represents a social relation between two users [64]. We extend this model by associating three distinct quantities with every user. (1) A user has a storage cost, which is the monetary cost for storing one replica of her data (e.g., profile, statuses) in the cloud for one billing period. (2) Similarly, a user has a traffic cost, which is the monetary cost during a billing period because of the inter-cloud traffic. As mentioned earlier, due to social locality, in our settings the inter-cloud traffic only involves writes (e.g., post tweets, leave comments). We do not consider intra-cloud traffic, no matter read or write, as it is free of charge $[1,6]$. (3) A user has a sorted list of clouds for the purpose of QoS, as will be described in Section 3.2.5.

Fig. 3.1 is an example where 11 users are hosted by 3 clouds. Black circles represent each user's master replica, and red ones represent the slave replicas of neighbors to ensure social locality. Solid lines are social relations and dotted arrows are the synchronization traffic. Within each black circle, the value on the top is the storage cost of a user, and the value at bottom is the traffic cost. For this placement, we can find the total storage cost is 330 and the total inter-cloud traffic cost is 50 . 


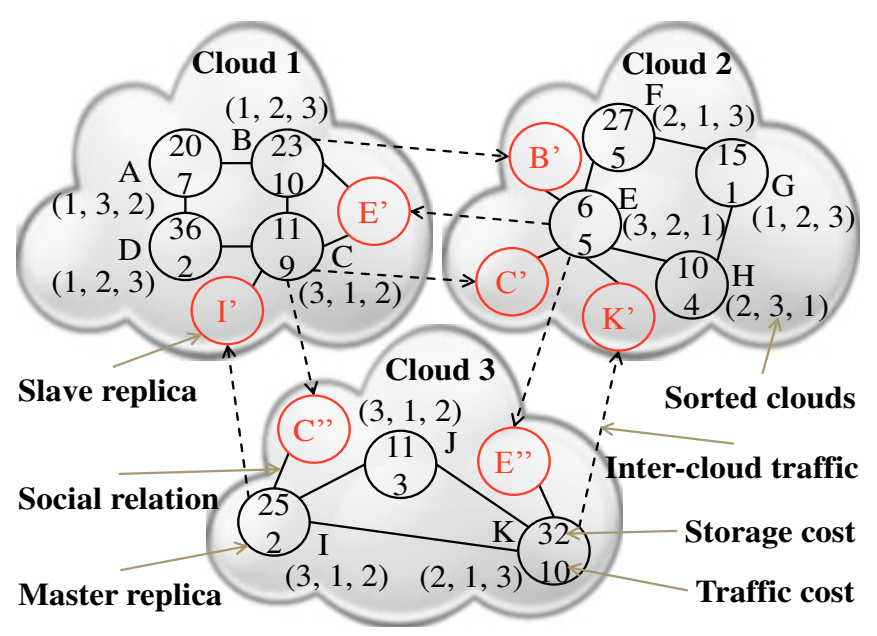

Figure 3.1: Storage and inter-cloud traffic cost

Besides the cost described above, note that the read/write operations themselves are usually charged based on the number of operations performed [1]. As we require the social locality for every user, the number of read operations performed by a user on all replicas of hers and her friends neither depends on the number of the replicas nor on the placement of the replicas. The charging for read operations is thus out of the scope of our optimization of replica placement. In contrast, the number of the write operations performed by a user on all replicas of hers and her friends replies on the number and the placement of the replicas. Fortunately, its charging can be included just as part of a user's traffic cost. For example, let $\tau_{u}=w_{u} T$ denote user $u$ 's traffic cost, where $w_{u}$ is the number of writes performed on $u$ 's data and $T$ is the average traffic cost incurred by a single write. Then, one can include the cost charged for a single write into $T$ so that optimizing the total inter-cloud traffic cost by our model can actually optimize the sum of the traffic and the read/write operations cost.

We make further assumptions. When calculating the costs, we assume that all clouds have the same billing prices. In reality, resource usage of clouds from different providers or at different locations may be charged at different prices. Such cases can be easily addressed by associating a proper weight with each cloud in our model, and our proposed algorithm, as shown later, can also straightforwardly adapt to these cases. We also assume that each cloud can provide "infinite" resources on demand to an OSN service provider, a guarantee often provided by a cloud provider to its customers.

\subsubsection{Modeling the Redistribution Cost}

An important part of our cost model is the cost incurred by the optimization mechanism itself, which we call the redistribution cost. We generally envisage that an optimization mechanism to be devised optimizes the cost by moving data 
across clouds to optimum locations, thus incurring such cost. The redistribution cost is essentially the inter-cloud traffic cost, but in this chapter we use the term inter-cloud traffic to specifically refer to the inter-cloud write traffic for maintaining replica consistency, and treat the redistribution cost separately.

We expect that the optimization is executed at a per-billing-period granularity (e.g., per-month) for the following reasons. First, this frequency is consistent with the billing period, the usual charging unit for a continuously running and long-term online service. The OSN provider should be enabled to decide whether to optimize the cost for each billing period, according to her monetary budget and expected profit, etc. Also, applying any cost optimization mechanism too frequently may fail the optimization itself. At the time of writing this chapter, the real-world price of inter-cloud traffic for transferring some data once is quite similar to that of storing the same amount of data for an entire billing period $[1,6]$. As a result, moving data too frequently can incur more redistribution cost that can hardly be compensated by the saved storage and inter-cloud traffic cost. Without loss of generality, we assume that the optimization mechanism is applied only once at the beginning of each billing period, i.e., the redistribution cost only occurs at the beginning of every billing period.

\subsubsection{Approximating the Total Cost}

Consider the social graph in a billing period. As it may vary within the period, we denote the final steady snapshot of the social graph in this period as $G^{\prime}=\left(V^{\prime}, E^{\prime}\right)$, and the initial snapshot of the social graph at the beginning of this period as $G=(V, E)$. Thus, the graph $G$ experiences various changescollectively called $\Delta G$ - to become $G^{\prime}$, where $\Delta G=(\Delta V, \Delta E), \Delta V=V^{\prime}-V$, and $\Delta E=E^{\prime}-E$.

Now consider the total cost incurred during a billing period. Denoting the total cost, the storage plus the inter-cloud traffic cost, the maintenance cost, and the redistribution cost during a period as $\Psi, \Phi(\cdot), \Omega(\cdot)$, and $\Theta(\cdot)$, respectively, we have

$$
\Psi=\Phi(G)+\Phi(\Delta G)+\Omega(\Delta G)+\Theta(G) .
$$

The storage cost in $\Phi(G)+\Phi(\Delta G)$ is for storing users' data replicas, including the data replicas of existing users and of those who just join the service in this period. The inter-cloud traffic cost in $\Phi(G)+\Phi(\Delta G)$ is for propagating all users' writes to maintain replica consistency. The redistribution cost $\Theta(G)$ is the cost of moving data across clouds for optimization; it is only incurred at the beginning of a period, following our previous assumption. There is also some underlying cost $\Omega(\Delta G)$ for maintenance, described as follows.

The maintenance cost $\Omega(\Delta G)$ is used to capture the cost spent on handling OSN changes. When a new user joins the OSN service, the service selects a 


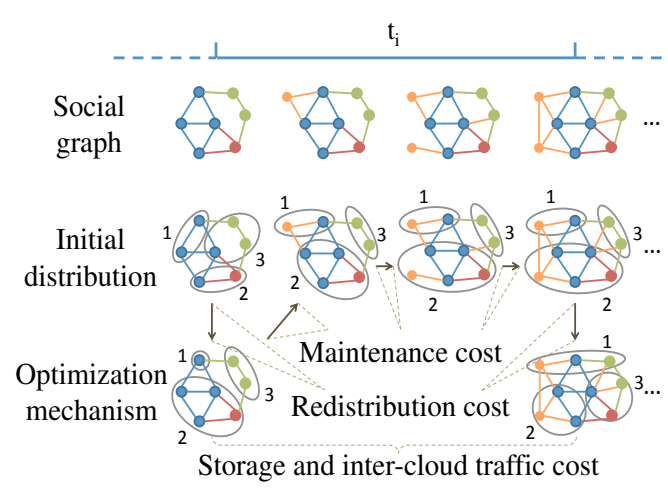

Figure 3.2: Different types of costs

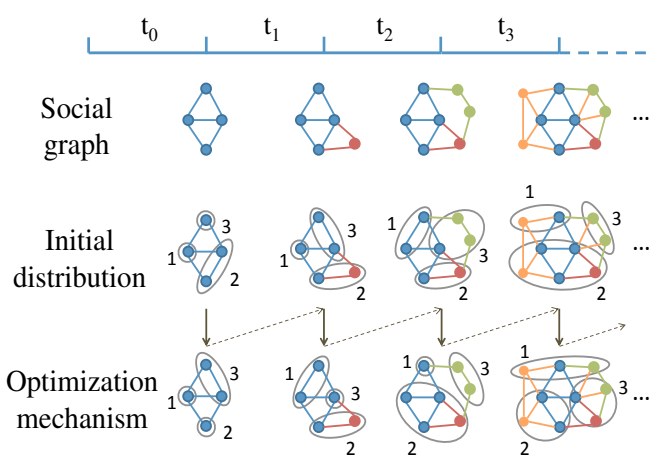

Figure 3.3: Cost over time

cloud and places this user's data there. Some time later after this initial placement and no later than the end of the current billing period, the OSN service must maintain social locality for this user and her neighbors, including creating new slave replicas on involved clouds as needed, causing the maintenance cost. However, in reality, $\Omega(\Delta G)$, as well as $\Phi(\Delta G)$, become negligible as the size of $\Delta G$ (i.e., $|\Delta V|$ ) becomes much smaller than that of $G$ (i.e., $|V|)$ when the OS$\mathrm{N}$ user base reaches a certain scale. Existing research observes that real-world OSNs usually have an S-shape growth $[19,33]$. As the user population becomes larger, the increment of the total number of users or social relations will decay exponentially $[46,96]$. Let us look at the monthly growth rate (i.e., $|\Delta V| /|V|$ ) in some real examples. According to Facebook [4], after its user population reached 58 million by the end of 2007, it grew with an average monthly rate below $13 \%$ through 2008 and 2009, a rate below 6\% through 2010, and then a rate below $4 \%$ until the end of 2011 when it reached 845 million. For Twitter, its average monthly growth rate was less than $8 \%$ in most months between March 2006 and September 2009 [10]; similar rates were observed for YouTube and Flickr [63].

Therefore, we derive an approximated cost model as

$$
\Psi \approx \Phi(G)+\Theta(G)
$$

which we will focus on throughout the rest of this chapter. Note that calculating $\Psi$ requires the storage cost and the traffic cost of each user in $G$. For any cost optimization mechanism that runs at the beginning of a billing period, an estimation is required to predict each user's costs during this billing period. Let's for now deem that the costs can be estimated and known. We defer the discussion on cost estimation to Section 3.5.1.

Fig. 3.2 and 3.3 illustrate different types of costs during a single billing period and consecutive billing periods. The numbers in the figures are the cloud IDs. Slave replicas are not drawn for the ease of presentation.

Note that, for the initial data placement, the OSN service may use various pre-specified strategies to choose a cloud, such as choosing the one with the 
lowest access latency for the user [80,82]. At this time point the OSN cannot determine an optimum cloud in terms of cost for a new user, as it knows neither the user's storage cost (except for a certain reserved storage such as storing a profile with pre-filled fields) nor her traffic cost for the current billing period. We assume that an OSN places a new user's data on her most preferred cloud.

\subsubsection{Modeling QoS and Data Availability}

Sorting clouds: Among all clouds, one cloud can be better than another for a particular user in terms of certain metric(s) (e.g., access latency, security risk). For instance, concerning access latency, the best cloud to host the data requested by a user is likely the geographically closest cloud to that user. Given $N$ clouds and $|V|$ users, with cloud IDs $\{1, \ldots, N\}$ (denoted as $[N]$ hereafter) and user IDs $\{1, \ldots,|V|\}$ (denoted as $[|V|]$ hereafter), clouds can be sorted for user $u$ as $\overrightarrow{c_{u}}=\left(c_{u 1}, c_{u 2}, \ldots, c_{u N}\right)$, where $c_{u i} \in[N], \forall i \in[N]$. For any cloud $c_{u i}, c_{u j}, i<j$, we deem that $c_{u i}$ is more preferred than $c_{u j}$; in other words, placing user $u$ 's data on the former provides better service quality to this user than the latter. The clouds $\left\{c_{u 1}, c_{u 2}, \ldots, c_{u j}\right\}, \forall j \in[N]$ are thus the $j$ most preferred clouds of user $u$, and the cloud $c_{u j}$ is the $j$ th most preferred cloud of user $u$. This sorting approach provides a unified QoS abstraction for every user while making the underlying metric transparent to the rest of the QoS model.

Defining QoS: We define the QoS of the entire OSN service as a vector $\vec{q}=(\vec{q}[1], \vec{q}[2], \ldots, \vec{q}[N])$, with

$$
\vec{q}[k]=\frac{1}{|V|} \sum_{u=1}^{|V|} \sum_{j=1}^{k} f_{u}\left(m_{u}, j\right), \forall k \in[N],
$$

where $m_{u}$ denotes the ID of the cloud that hosts the master data replica of user $u, f_{u}(i, j)$ is a binary function that equals to 1 if cloud $i$ is user $u$ 's $j$ th most preferred cloud but 0 otherwise. Therefore, $\vec{q}[k]$ is the ratio of users whose master data are placed on any of their respective $k$ most preferred clouds over the entire user population. This CDF-style vector allows OSN providers to describe QoS at a finer granularity.

Let us refer back to Fig. 3.1 as an example, where the vector associated with each circle represents the sorted cloud IDs for the corresponding user. We see that out of all the 11 users, 7 are hosted on their first most preferred cloud, 10 on either of their two most preferred clouds, and all users on any of their three most preferred clouds. Thus, the QoS is $\vec{q}=\left(\frac{7}{11}, \frac{10}{11}, 1\right)$.

Comparing QoS: There can be different data placements upon clouds. Each may result in a different corresponding QoS vector. For two QoS vectors $\vec{q}_{a}$ and $\vec{q}_{b}$ representing two placements respectively, we deem that the former placement provides QoS no better than the latter, i.e., $\vec{q}_{a} \leq \vec{q}_{b}$, if every element 
of the former vector is no larger than the corresponding element of the latter, i.e., $\vec{q}_{a}[k] \leq \vec{q}_{b}[k], \forall k \in[N]$.

QoS requirement: We model the QoS requirement as two vectors $\vec{Q}_{l}$ and $\vec{Q}_{u}, \vec{Q}_{l} \leq \vec{Q}_{u}$ that serve as a lower bound and an upper bound, respectively. In order to meet the QoS requirement, a data placement must have a QoS $\vec{q}$ that meets $\vec{Q}_{l} \leq \vec{q} \leq \vec{Q}_{u}$. Specified by the OSN provider, $\vec{Q}_{l}$ captures the worst QoS that can be tolerated and $\vec{Q}_{u}$ captures the best QoS that can be provided. Note that we do not require $\vec{Q}_{u}$ represent the placement of every user's data on her first most preferred cloud. $\vec{Q}_{u}$ can be set as any valid QoS vector, subject to the OSN provider's customized policies and considerations.

As an example, let us see how $\vec{Q}_{l}$ can express " $80 \%$ of all users must access data in no more than $200 \mathrm{~ms}$." In this case, clouds are sorted according to access latency for every user. For any user $u$, we can calculate that only putting her master data replica on any of her $n_{u}, n_{u} \in[N]$ most preferred clouds can grant her the latency of no more than $200 \mathrm{~ms}$. By denoting $n_{\min }=\min \left\{n_{u} \mid \forall u \in\right.$ $[|V|]\}$, this requirement can thus be expressed by setting $\vec{Q}_{l}\left[n_{\text {min }}\right]=0.8$. If $n_{\text {min }} \neq 1$, then $\vec{Q}_{l}[k], \forall k \in\left\{1, \ldots, n_{\text {min }}-1\right\}$ can be set as any value as long as $0 \leq \vec{Q}_{l}\left[k_{1}\right] \leq \vec{Q}_{l}\left[k_{2}\right] \leq 0.8,1 \leq k_{1}<k_{2}<n_{\text {min }}$. In fact, $\vec{Q}_{l}$ can express any fine-grained requirement such as " $95 \%$ of users' access must be satisfied within $500 \mathrm{~ms}, 80 \%$ be satisfied within $200 \mathrm{~ms}$ and $65 \%$ be satisfied within $90 \mathrm{ms."}$

Data availability requirement: An OSN provider specifies the data availability requirement by indicating the minimum number of every user's slave replicas. We denote it using a number $R, R \in\{0, \ldots, N-1\}$, where $N$ is the number of clouds. In order to meet the data availability requirement, each user must maintain slave replicas no fewer than $R$. If the number of a user's slave replicas to maintain social locality is no smaller than $R$, the data availability requirement for this user has already been met and this user does not have to own more slaves; in contrast, besides the slaves to maintain social locality, if a user does not have enough slaves to meet the data availability requirement, then this user must have more slaves to ensure that the total number of her slaves is equal to $R$.

\subsection{Problem}

\subsubsection{Problem Formulation}

With the models defined in Section 3.2, we are interested in the following problem: given an existing data placement upon $N$ clouds of OSN $G(V, E)$ with $|V|$ users, find out the optimal data placement with the minimal total cost-i.e., the sum of the storage and inter-cloud traffic cost $\Phi(G)$ and the redistribution cost $\Theta(G)$ for implementing this optimal placement from the existing placement- 
while ensuring QoS and data availability meet pre-defined requirements.

We introduce the following notations in order to formulate the problem. $m_{u i}$ and $s_{u i}$ are binary decision variables. The former equals to 1 if in the optimal placement user $u$ 's master replica is placed on cloud $i$, and 0 otherwise. The latter equals to 1 if in the optimal placement $u$ has a slave replica placed on cloud $i$, and 0 otherwise. $m_{u i}^{\prime}$ and $s_{u i}^{\prime}$ are also binary, and are counterparts of $m_{u i}$ and $s_{u i}$ respectively in the existing placement. $\mu_{u}$ is the storage cost for storing one master or slave replica of user $u$. $\tau_{u}$ is the traffic cost for synchronizing one slave replica of user $u$. $\beta$ is the coefficient for converting the storage cost of a replica to the redistribution cost of moving this replica across clouds. $e_{u v} \in E$ if user $u$ and user $v$ are neighbors. $f_{u}(i, j)$ is a binary function indicating whether cloud $i$ is user $u$ 's $j$ th most preferred cloud (as introduced in Section 3.2.5). The

QoS requirement is given by two vectors $\vec{Q}_{l}$ and $\vec{Q}_{u}$, and the data availability requirement is given by a number $R$. We formulate the problem as follows.

\section{minimize}

$$
\Phi(G)+\Theta(G)
$$

where

$$
\begin{gathered}
\Phi(G)=\sum_{u=1}^{|V|}\left(\mu_{u} \sum_{i=1}^{N}\left(m_{u i}+s_{u i}\right)+\tau_{u} \sum_{i=1}^{N} s_{u i}\right) \\
\Theta(G)=\sum_{u=1}^{|V|}\left(\beta \mu_{u} \sum_{i=1}^{N}\left(\max \left\{\left(m_{u i}+s_{u i}\right)-\left(m_{u i}^{\prime}+s_{u i}^{\prime}\right), 0\right\}\right)\right)
\end{gathered}
$$

subject to

$$
\begin{gathered}
\sum_{i=1}^{N} m_{u i}=1, \forall u \in[|V|] \\
m_{u i}+s_{u i} \leq 1, \forall u \in[|V|], \forall i \in[N] \\
m_{v j}+s_{v j}=1, j=\sum_{i=1}^{N}\left(i m_{u i}\right), \text { if } e_{u v} \in E, \forall u, v \in[|V|] \\
\sum_{i=1}^{N} s_{u i} \geq R, \forall u \in[|V|] \\
\vec{Q}_{l}[k] \leq \frac{1}{|V|} \sum_{u=1}^{|V|} \sum_{j=1}^{k} f_{u}\left(\sum_{i=1}^{N}\left(i \cdot m_{u i}\right), j\right) \leq \vec{Q}_{u}[k], \forall k \in[N]
\end{gathered}
$$

Constraint (3.1) ensures that every user has a single master replica. Constraint (3.2) ensures that no master and slave replicas of the same user are co-located on a common cloud. Constraint (3.3) ensures the social locality. Constraint (3.4) ensures the data availability. Constraint (3.5) ensures that the 
QoS of the data placement meets the QoS requirement. All constraints apply to both the existing data placement and the optimal placement. Here we do not write the existing case for the ease of presentation. Our cost optimization problem is NP-hard.

\subsubsection{Contrast with Existing Problems}

To the best of our knowledge, existing problems that are most related to our problem defined above are likely the MIN_REPLICA [73] problem and the graph partitioning [52] problem. Here we highlight why they fail to capture our scenario in this chapter.

The MIN_REPLICA problem minimizes the total number of replicas of users' data while maintaining social locality, load balance across partitions, and a given redundancy. Compared with our problem, it falls short in the following aspects. First, it does not consider QoS. It balances the number of master replicas across servers within a single cloud, while we aim to place masters across multiple clouds in order to ensure QoS meet a pre-defined requirement. Second, it does not consider data redistribution, as it targets intra-cloud placement where redistribution is free. In our case, the redistribution cost is inter-cloud and is an important part of our objective. Third, it does not model the storage cost and the traffic cost of each user. It minimizes the total number of replicas, while we aim to minimize the total monetary cost that an OSN service provider has to pay to the cloud provider.

The graph partitioning problem minimizes the total amount of communication volume or the total weights of edges across partitions while maintaining load balance. Compared with our problem, it has no notion of social locality at all, neither does it capture the QoS and the data availability requirements.

\subsubsection{NP-Hardness Proof}

We prove the NP-hardness of our cost optimization problem by restriction. Specifically, we show that the MIN_REPLICA problem, which has been proved NP-hard [73], is contained by our problem as a special case. Firstly, let $\beta=0$ and let $\mu_{u}=0, \tau_{u}=1, \forall u \in[1, M]$. This makes the objective of our cost optimization problem the same as that of MIN_REPLICA, both minimizing the total number of slave replicas. Secondly, let the list of the sorted clouds of every user be identical, i.e., $f_{u}(i, j)=f_{v}(i, j), \forall u, v \in[1, M], \forall i, j \in[1, N]$, and let $\vec{Q}_{l}$ and $\vec{Q}_{u}$ satisfy $\vec{Q}_{l}[k]=\vec{Q}_{u}[k]=\frac{k}{N}, \forall k \in[1, N]$. This makes Constraint (3.5) of our problem equivalent to the load balance constraint of MIN_REPLICA, both maintaining an equal number of master replicas across partitions. Thirdly, note that all other constraints of our problem are the same as their counterparts of MIN_REPLICA. Hence, MIN_REPLICA is a case of our problem under the 
above settings. Due to the NP-hardness of MIN_REPLICA, our cost optimization problem is NP-hard.

\subsection{Algorithm}

Our cost optimization problem is an Integer Programming (IP) problem [68]. The huge user population of real-world OSN services translates into a huge number of decision variables, and the NP-hardness of our problem makes it impossible to be efficiently solved by existing general-purpose IP solvers. We thus seek practical heuristics. We propose cosplay, an optimization algorithm that iteratively swaps the roles of master and slave replicas on different clouds in order to adjust an existing placement towards the optimal placement.

\subsubsection{Observations of Role-Swaps}

Our algorithm is inspired by three observations below when swapping a master replica and a slave replica of a user. In this what we call a role-swap process, the master replica becomes a slave replica and the slave becomes the master. We use Fig. 3.4 and Fig. 3.5 to illustrate our observations, where lines and circles in these figures have the same meanings as in Fig. 3.1, and each user has 1 unit of storage cost and 1 unit of traffic cost. Note that while symbols like $u, v$ are supposed to denote users throughout this chapter, we also use them to denote the master replicas of the corresponding users in the figures here.

Observation 1: Role-swap can lead to possible cost reduction. Fig. 3.4 is a simple example with 4 users hosted by 3 clouds. For user $u$, we may choose to swap the roles of replica $u$ with replica $u^{\prime}$ (as in Fig. 3.4(b)), or swap the roles of replica $u$ with replica $u^{\prime \prime}$ (as in Fig. 3.4(c)), while maintaining the social locality. Before the swap as in Fig. 3.4(a), there are 10 units of replica storage and 6 units of inter-cloud traffic. After the swap, as either in Fig. 3.4(b) or in Fig. 3.4(c), there are 9 units of replica storage and 5 units of inter-cloud traffic. We thus save 1 unit of replica storage and 1 unit of inter-cloud traffic by paying 1 unit of redistribution cost (caused by copying the replica $v_{3}$ to create a new replica $v_{3}^{\prime}$ in Fig. 3.4(b), or $v_{3}^{\prime \prime}$ in Fig. 3.4(c)). Overall, we can achieve 1 unit of cost reduction.

Observation 2: Because of the QoS requirement, not every role-swap is feasible. When multiple role-swaps for a user are available, we must choose the one(s) meeting QoS requirements. The two different role-swap choices taken in Fig. 3.4(b) and in Fig. 3.4(c) result in the same amount of cost reduction. Let us suppose every cloud has an ID and every user has a sorted list of preferred clouds as shown in the figure. Before swap, $\vec{q}=(0.75,0.75,1)$. If the QoS requirement is given by $\vec{Q}_{u}=(1,1,1)$ and $\vec{Q}_{l}=(0.5,0.75,1)$, then we should choose Fig. 3.4(c) 
instead of Fig. 3.4(b), because the QoS of the former is $\vec{q}=(0.5,0.75,1)$, which still meets the QoS requirement, and the QoS of the latter is $\vec{q}=(0.5,0.5,1)$, which violates the QoS requirement.

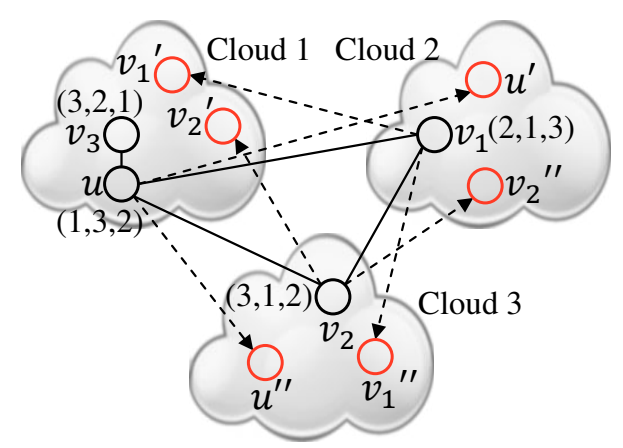

(a) Before swap

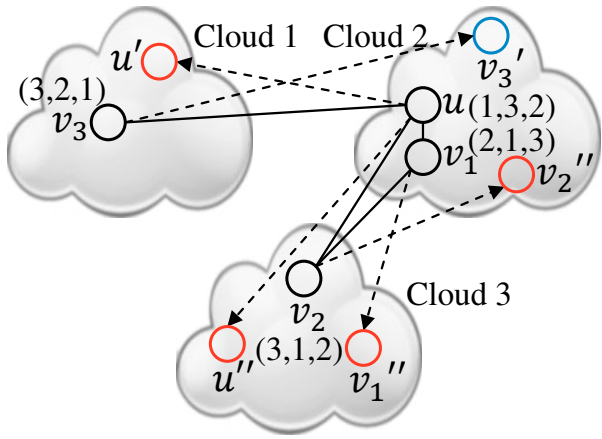

(b) Swap $u$ with $u^{\prime}$

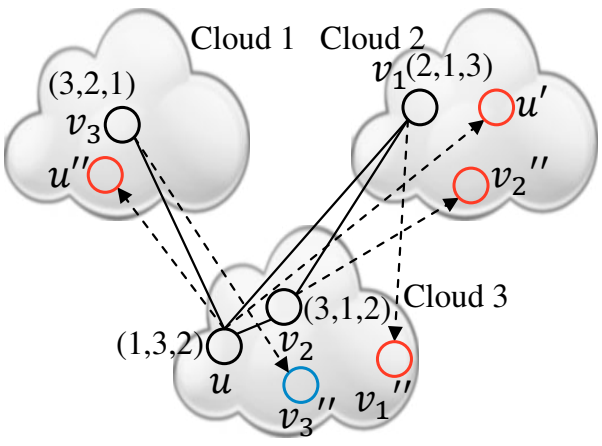

(c) Swap $u$ with $u^{\prime \prime}$

Figure 3.4: Role-swap of $u$

Observation 3: Every user needs to have slave replicas no fewer than a given number, which can be maintained when performing role-swaps. We use Fig. 3.5 for illustration, where 5 users are hosted on 3 clouds, and for simplicity, we do not show the sorted lists of cloud IDs. Suppose in this example, the required minimum number of slaves of every user is 1 . After maintaining social locality, users $u, v_{1}, v_{2}$, and $v_{4}$ already meet this requirement, but user $v_{3}$ still needs a slave replica for data availability, i.e., $v_{3}^{\prime}$ in this figure. Now we swap the roles of replicas $u$ and $u^{\prime \prime}$ for cost reduction. After this swap, note that the slave replica $v_{4}^{\prime}$ is not needed for maintaining the social locality of $u$, but it is still needed to satisfy the data availability of user $v_{4}$. Therefore, in this case, the cost reduction is 4 units, instead of 6 units if we remove $v_{4}^{\prime}$.

\subsubsection{Algorithm Based on Role-Swaps}

Inspired by the above three observations, we employ a series of role-swaps to maximize the total cost reduction while maintaining data availability and en- 


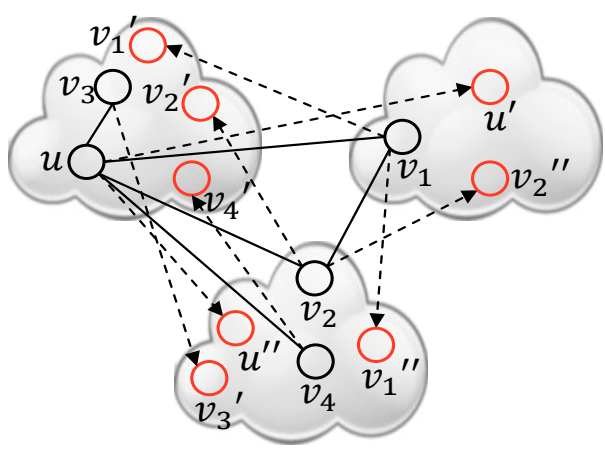

(a) Before swap

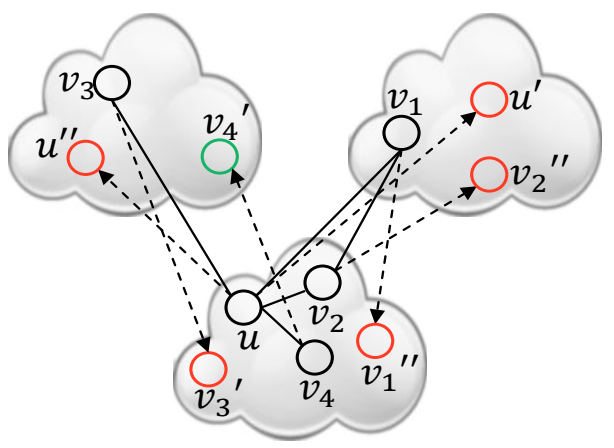

(b) Swap $u$ with $u^{\prime \prime}: v_{4}^{\prime}$ is kept

Figure 3.5: Role-swap of $u$

suring QoS requirements. Our algorithm follows a greedy approach in using role-swaps and we require that every applied role-swap reduce cost. The more cost reduction each role-swap has and the more role-swaps are applied, the more total cost reduction we can achieve. Note that our algorithm computes a better placement, and it does not physically manipulate data. When our algorithm terminates, data are role-swapped or moved (in the case of redistribution) from existing locations to new locations in order to implement the new placement output by our algorithm.

We describe our cosplay algorithm as follows. The procedures of single roleswaps and double role-swaps are repeated one after the other until neither of them can be further executed or until a specified number of iterations are reached.

Single role-swaps: In each iteration, select a user randomly. For each feasible role-swap between this user's master and one of her slaves, calculate the cost reduction. Then, choose the role-swap with the largest cost reduction and apply it. Repeat this until no further cost can be reduced.

Double role-swaps: In each iteration, select a user randomly, and pair this user with each of her neighbors whose master is on a different cloud. For each of such pairs, first check if the following pair of role-swaps is feasible: one between the selected user's master and her slave on the neighbor's cloud and the other between the neighbor's master and her slave on the selected user's cloud. If feasible, calculate the cost reduction of these two role-swaps. Then, choose the pair with the largest cost reduction and apply the two role-swaps. Repeat this until no further cost can be reduced.

Whether a single role-swap or a double role-swap, three basic but non-trivial operations of cosplay are needed: determining whether it is feasible, calculating its cost reduction, and swapping the roles of involved replicas. We elaborate how to efficiently achieve these operations below. 


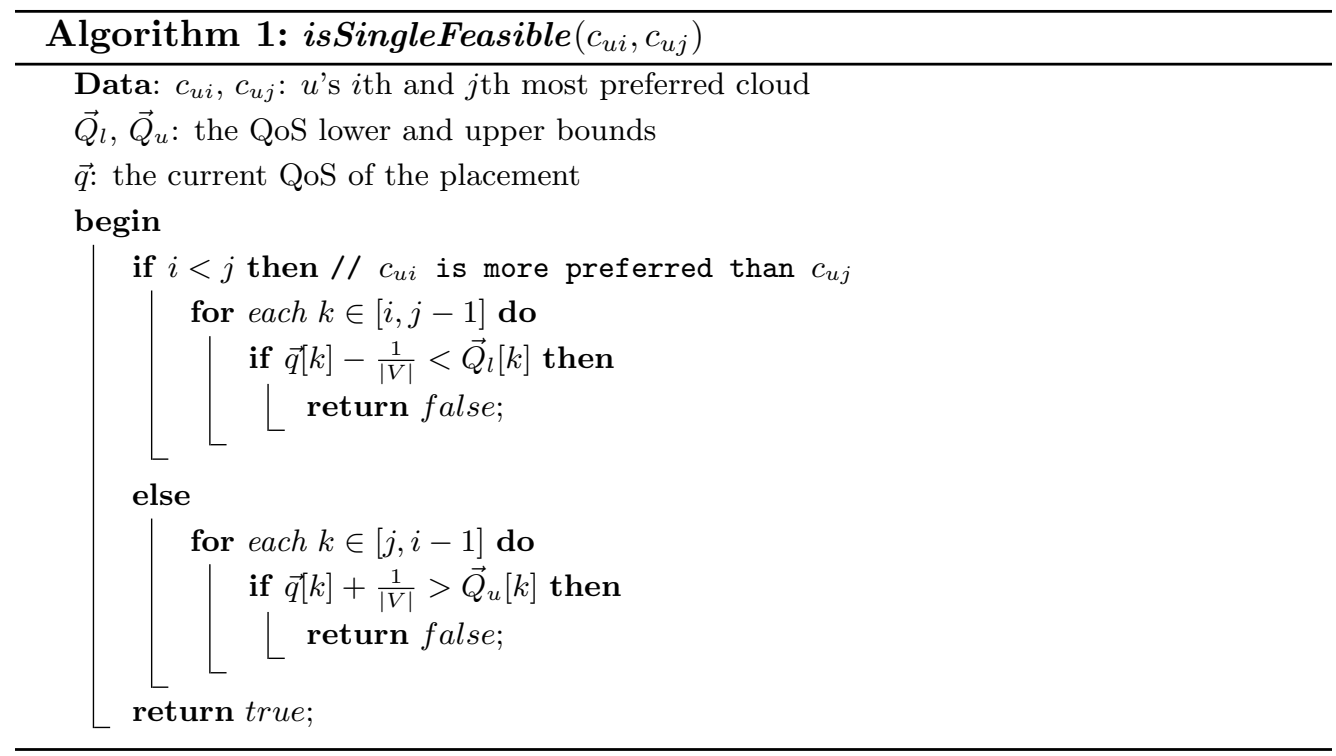

\subsubsection{Determining Feasibility}

Algorithms 1 and 2 determine the feasibilities of a single role-swap and a double role-swap, respectively. Algorithm 1 checks whether applying a role-swap would make the current QoS out of the range specified by the QoS lower bound and upper bound. In Algorithm 1, user u's master and slave replicas are on cloud $c_{u i}$ and $c_{u j}$ respectively. Algorithm 2 invokes Algorithm 1, where users $u$ and $v$ are selected, with their masters on cloud $c_{u i}$ and $c_{v i}$ and slaves on $c_{u j}$ and $c_{v j}$, respectively. Note that applying one role-swap can change the current QoS, and the feasibility of the next role-swap must be considered based on the new QoS. We do not show the function $\boldsymbol{a d j u s t} \boldsymbol{Q o S}\left(c_{u i}, c_{u j}\right)$ as it is very simple, adjusting $\vec{q}$ in a way similar to Algorithm 1 .

\subsubsection{Calculating Cost Reduction}

Algorithms 3 and 4 specify the calculations of the cost reduction of a single role-swap and a double role-swap, respectively. Here we highlight three of our insights about Algorithm 3 as follows.

Local computation: To calculate the cost reduction for a role-swap between user $u$ 's master and her slave, an intuitive option would be calculating the difference between the total cost of the old placement (i.e., the one before applying the role-swap) and that of the new placement (i.e., the one after applying the role-swap). However, doing so involves accessing every user and calculating the total cost twice, which can cause considerable computation overhead given the number of role-swaps we may have with a large social graph. In fact, we observe that the cost reduction can be calculated by accessing only local information. The cost reduction only depends on the storage and traffic cost of user $u$ and her 


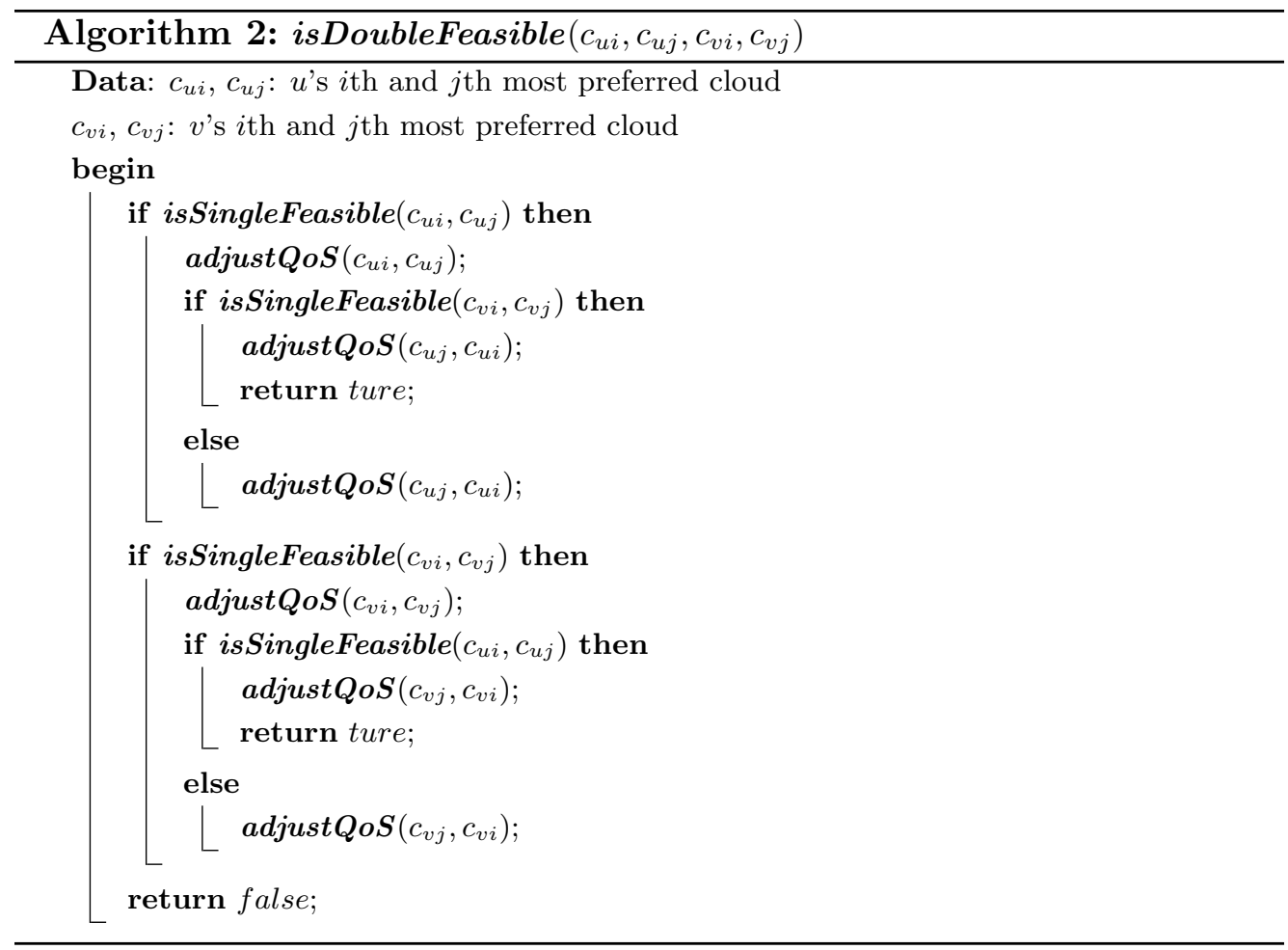

neighbors, and the locations of their replicas in the old placement and the new placement. If on user $u$ 's master cloud we store a slave replica of her neighbor $v$ to maintain the social locality for $u$, and if $v$ has no other neighbors of her own on this cloud, a role-swap between user $u$ 's master and her slave will cause this slave replica of $v$ useless, and this replica is thus a candidate for elimination (whether it can be eliminated further depends on data availability as described below). Local computation is sufficient for calculating the reduced cost.

Redistribution cost: The cost of redistribution incurred by a role-swap depends on the new placement where this role-swap is applied, and the existing placement which is the input to our cosplay algorithm. In the new placement, when a slave needs to be created on a cloud for social locality, we check if it did not exist on the cloud in the existing placement. If so, the cost of creating this slave is added to the redistribution cost incurred by this role-swap. Similarly, when a slave is to be removed as it is no longer needed, we check whether this slave existed on its current cloud in the existing placement. If not, this slave was created by a previous role-swap and the incurred redistribution cost of creating this slave has already been counted. We thus subtract this cost from the redistribution cost of the role-swap.

Data availability: Whether to remove a slave or not does not only depend on social locality, but also on the data availability requirement. Creating slaves is always fine because it never violates the data availability requirement. We must ensure that if we remove a slave replica, the number of slaves of this user is still 


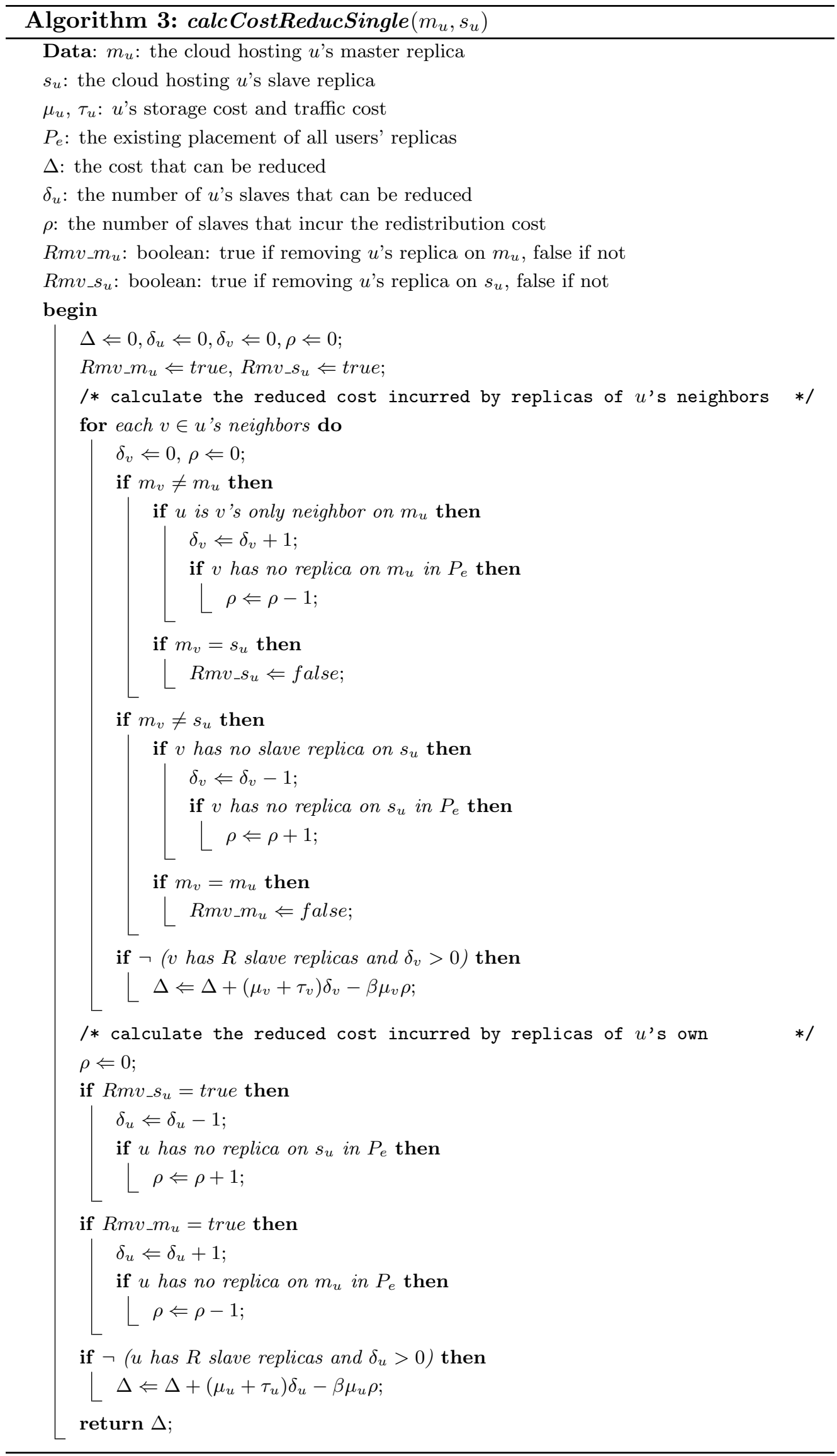



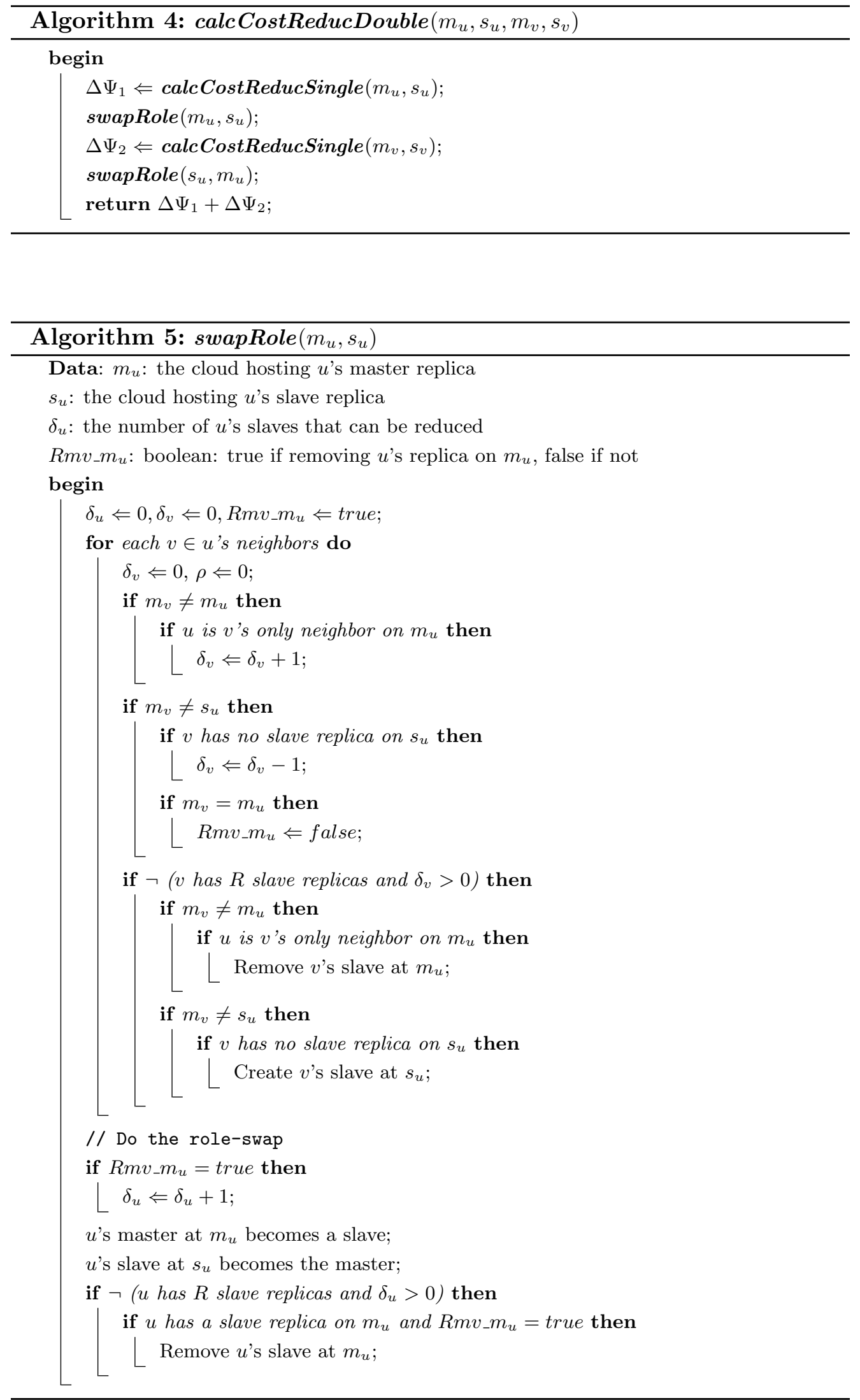
no fewer than the pre-specified number, thus maintaining the data availability for this user. If we cannot remove a slave due to data availability, this user should not be considered when calculating cost reduction of a role-swap that involves this user, and the slave is also not touched when performing the role-swap.

\subsubsection{Swapping Roles of Replicas}

Algorithm 5 describes the operation of swapping the roles of a user $u$ 's master on cloud $m_{u}$ and her slave on cloud $s_{u}$. Swapping the roles does not simply involve $u$ 's replicas alone; instead, it may also involve removing or creating her neighbors's slave replicas due to social locality and data availability. The flow of this algorithm shares some similarities with that of Algorithm 1, as calculating the cost reduction of a role-swap before it is performed is actually simulating how the cost would be affected if the role-swap was performed.

\subsection{Evaluations}

We carry out extensive evaluations by placing real-world Twitter data over 10 clouds all across the US. We demonstrate significant one-time and accumulated cost reductions compared to existing approaches, while always ensuring QoS and data availability requirements. By varying the experimental settings, we also investigate the complex trade-off among cost, QoS, and data availability.

\subsubsection{Data Preparation}

Collecting data: We crawled Twitter during March and April 2010 in a breadth-first manner, consistent with previous OSN crawls [64, 85]. We collected $3,117,553$ users with 23,883,149 social relations. For each user, we have her profile, tweets, and the list of followers. Among all the users, 1,157,425 users provide location information in their profiles.

Sorting clouds for each user: Due to the lack of publicly available dataset on latencies between OSN users and OSN sites, we cannot sort clouds for users in terms of latency. However, the geographic distance is widely used as an important QoS metric in previous work [29, 40]. With our Twitter dataset, we thus sort clouds for each user in terms of the real-world geographic distance between a user and the clouds.

We focus on users who are geographically located in the US. With the help of the database of US Board on Geographic Names [8] and Google Maps, we convert users' text locations to geo-coordinates (i.e., [latitude, longitude]). Out of all these users, we extract the largest connected component of 321,505 users with 3,437,409 social relations as the input to our evaluations. We then select 10 cities all across the US as cloud locations: Seattle (WA), Palo Alto (CA), Orem 


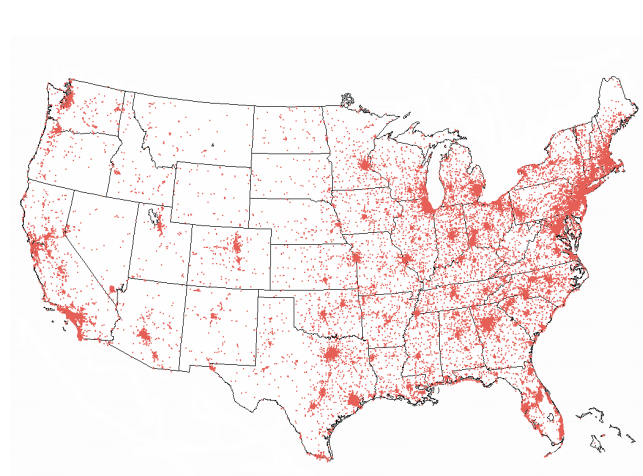

Figure 3.6: User locations

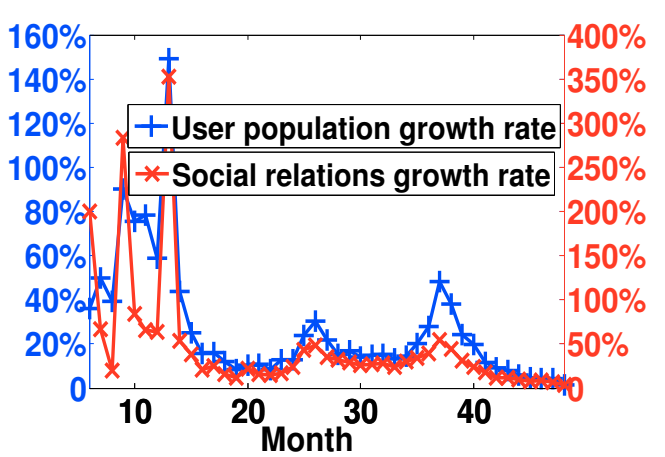

Figure 3.7: Monthly growth rate

(UT), Chicago (IL), San Antonio (TX), Lansing (MI), Alexandria (LA), Atlanta (GA), Ashburn (VA), and New York (NY). All these locations have real-world cloud data centers [3]. Afterwards, we sort all clouds for each user in terms of the respective geographic distance between a user and the clouds. Fig. 3.6 plots the locations of all the 321,505 users.

Extracting monthly OSN snapshots: To evaluate cosplay for OSN with dynamics, we extract monthly graph snapshots out of our largest connected component. Under the assumption that each user publishes her first tweet and forms her social relations immediately after she joins the service, and with the time stamp of each tweet and the follower list of each user, we find that the earliest user in our largest connected component joined Twitter in March 2006 and the latest user joined Twitter in February 2010. We thus can extract 48 monthly social graphs. Fig. 3.7 shows the monthly growth rates of our 48 graphs. The user base growing at about $15 \%$ since the 16th month is a good approximation of the real-world OSN growth, as discussed in Section 3.2.

Obtaining monthly costs for each user: We calculate costs by multiplying the unit price with the data size, and use such calculated costs of each user for each of the 48 months as the ground truth. We use $\$ 0.125 / \mathrm{GB} / \mathrm{month}$ for storage cost and $\$ 0.12 / \mathrm{GB}$ for inter-cloud traffic cost [6]. With each tweet and its time stamp, we can easily find how much data a user publishes in a given month (i.e., the traffic size of that month), and how much data this user has accumulated by the end of a given month (i.e., the storage size of that month).

In real world when executing cosplay in consecutive billing periods, it needs an estimate of each user's costs for a period at the beginning of the period. Existing research tells that users' online activities are bursty and the inter-activity time is heavy-tailed [21, 84], making the estimation of future activities difficult, not to mention the estimation of the data size which is of our interest. However, our goal here is not pursuing the estimation accuracy, but implying the trend of the size of a user's produced data so that we can make a right decision of role-swap. We exploit the Exponentially Weighted Moving Average, a common approach for 
similar purposes, to estimate the number of activities in a future time period with the smoothing factor $\alpha=0.9$ to capture the burstiness of user activities. By multiplying it with the average data size of all previous activities, we obtain the estimation of the data size in a future time period. As a result, we obtain 48 monthly graphs with the estimated costs of each user for each month.

\subsubsection{Experimental Settings}

We run two groups of evaluations. In the first group, with our largest February 2010 social graph as input, we compare the costs and the QoS' of the data placements produced by the greedy method, the random method, SPAR, METIS and cosplay, respectively. We also investigate how the costs are influenced by the data availability requirement and by the QoS requirement. We ensure social locality for all approaches for fair comparison. The greedy method places every user's master on her first most preferred cloud. The random method assigns a user's master to a cloud randomly. For SPAR, we implement it ourselves, and we treat each social relation between two users as an edge creation event and create a random permutation of all events to produce the edge creation trace as input, following the method suggested in [73]. For METIS, there is an opensource implementation from its authors. We use its option of minimizing the inter-partition communication. We use each user's storage cost plus her traffic cost as the vertex size (in METIS' terminology) to create its input. For cosplay, we use the greedy method to produce an existing placement.

We vary the number of most preferred clouds that users use to place masters, and we also vary the QoS and the data availability requirements. We have 10 clouds sorted for every user. Besides the 10-clouds case, we also compare the cases when each user uses her 2, 4, 6, and 8 most preferred clouds for master placement, respectively. We vary the QoS requirement by varying the lower bound while keeping the upper bound fixed at $\vec{Q}_{u}=(1)$. Note that, when there are 10 clouds a QoS vector should have 10 elements, but we omit the consecutive 1 's at the end of a QoS vector for the ease of presentation. $\vec{Q}_{u}=(1)$ corresponds to the greedy placement and is the best QoS that can be provided. We vary $\vec{Q}_{l}$ by the following rule. Given the value of the first element (hereafter we call it the "first value" for brevity) of the $\vec{Q}_{l}$ vector and given the number of most preferred clouds that users use, we set the values of all other elements of $\vec{Q}_{l}$ by building a linear growth from its first value to 1 . For example, if the first value of $\vec{Q}_{l}$ is 0.5 and users use 2 most preferred clouds, then $\vec{Q}_{l}=(0.5,1)$. With the same first value, if users use 6 most preferred clouds, then $\vec{Q}_{l}=(0.5,0.6,0.7,0.8,0.9,1)$. We vary the data availability requirement by iterating $R$ from 0 to 9 . We set $\beta=1$, reflecting the fact that the cost of moving some data across clouds once is similar to that of storing the same data in the cloud for one month. 
In the second group of evaluations, with the inputs of our 48 monthly OSN snapshots with real-world costs and the other 48 monthly snapshots with estimated costs, we focus on the continuous cost reduction that can be achieved by cosplay, compared with the greedy method. For each month, we run greedy on the former, representing the real-world common practice of placing user's data on the closest cloud for lowest access latency. We run cosplay on the former to show the "ideal" cost reduction, assuming we know the exact costs of each user for each month at the beginning of every month. We also run cosplay on the latter, where replica locations are adjusted according to the estimated costs of each user, to show the effectiveness of our estimation approach. Note that cosplay runs only once at the beginning of every month. When new users join the system during a month, each user is still placed by the greedy method. When only using greedy, the total cost for each month is the sum of the storage and the inter-cloud traffic cost, plus the maintenance cost. When running cosplay, the total cost for each month additionally includes the redistribution cost. We use the same $\vec{Q}_{u}$ and $\beta$ settings as in our first group of evaluations and only consider the case where every user uses all 10 most preferred clouds. We set $R=0$ and the first value of $\vec{Q}_{l}$ to be 0.5 .

In the figures, the cost of every placement is normalized as the quotient of the placement divided by the standard cost, where the standard cost is the cost of the greedy placement with $R=0$. The storage cost is normalized by the standard storage cost, the inter-cloud traffic cost and the redistribution cost is normalized by the standard inter-cloud traffic cost, and the total cost is normalized by the standard total cost.

\subsubsection{Results on One-time Cost Reduction}

We note that, throughout Fig. 3.8 to 3.11 and in Fig. 3.14 and 3.15, the first value of $\vec{Q}_{l}$ is always set as 0.5 , and we vary $\vec{Q}_{l}$ in Fig. 3.12 and 3.13.

Fig. 3.8 compares the costs of the placements produced by different methods over all the 10 clouds with $R=0$. For all methods except cosplay, the total cost is the sum of its storage and inter-cloud traffic cost. For cosplay, the total cost additionally includes the redistribution cost. The greedy placement has moderate cost compared with random. Users who are geographically close to one another tend to have similar sorted lists of clouds. Thus, greedy can assign local users to the same nearby cloud and random tends to straddle local social relations across clouds. SPAR has less cost than greedy and random but more than METIS, indicating that minimizing the number of replicas cannot necessarily minimize the cost. Cosplay outperforms all others with total cost reductions of $59 \%, 66 \%$, $50 \%$ and $44 \%$, compared to greedy, random, SPAR and METIS, respectively.

Fig. 3.9 depicts the total cost of each method over 10 clouds as $R$, the mini- 


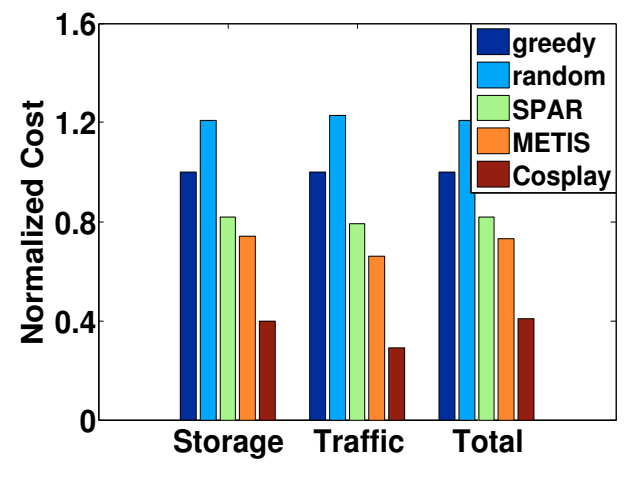

Figure 3.8: Cost comparison (I)

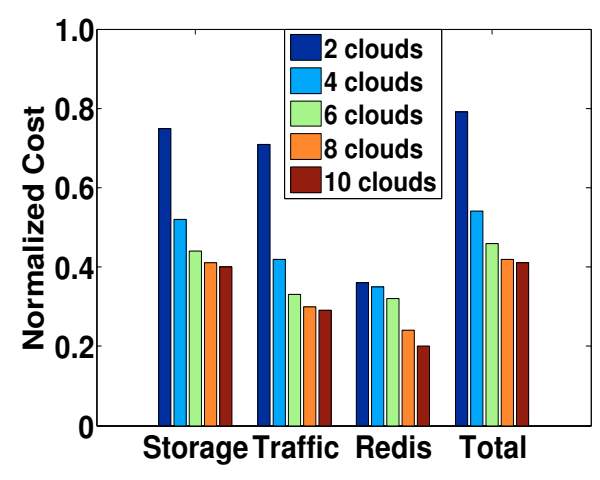

Figure 3.10: Cost of cosplay (I)

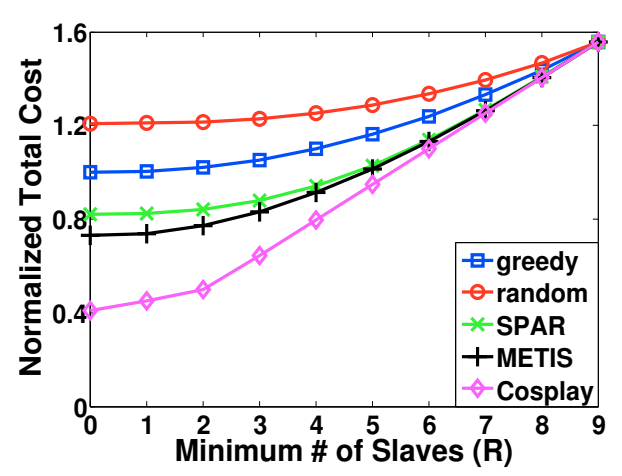

Figure 3.9: Cost comparison (II)

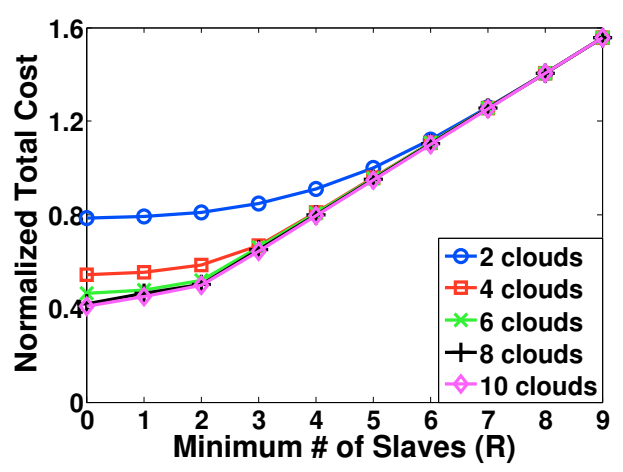

Figure 3.11: Cost of cosplay (II)

mum number of slave replicas required for every user to ensure data availability, varies. When $R$ is small, cosplay achieves more cost reduction via role-swaps and eliminates those slaves that are no longer needed for social locality. When it becomes larger, cosplay's advantages are decreasing, as it cannot eliminate some slaves as they are needed for data availability even if they are not needed for social locality. The room for optimization also becomes less. All methods turn to full replication when $R=9$.

Fig. 3.10 dissects the costs of the placements produced by cosplay on users' $2,4,6,8$, and 10 most preferred clouds in the case of $R=0$. As the number of involved clouds grows, the storage cost, the inter-cloud traffic cost and the total cost drops, as more optimization can be done if more clouds are available for each user. However, the redistribution cost also declines, indicating we pay less overhead to save more costs. The reason is, as the total number of feasible roleswaps grows, the number of users with negative redistribution cost also increases, dragging down the total redistribution cost as more role-swaps place masters on clouds where users do not have any replica in the existing placement.

Fig. 3.11 demonstrates the total cost of each cosplay placement as $R$ increases. No matter how $R$ changes, we observe that when users consider placing their masters on their most preferred clouds of more than 4 , the advantages of cosplay 


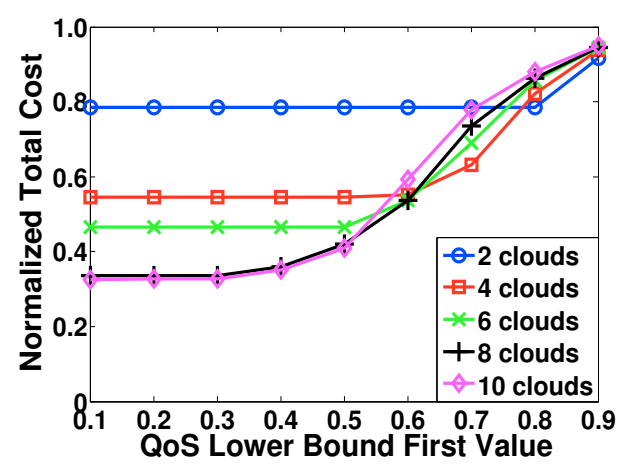

Figure 3.12: Cost of cosplay (III)

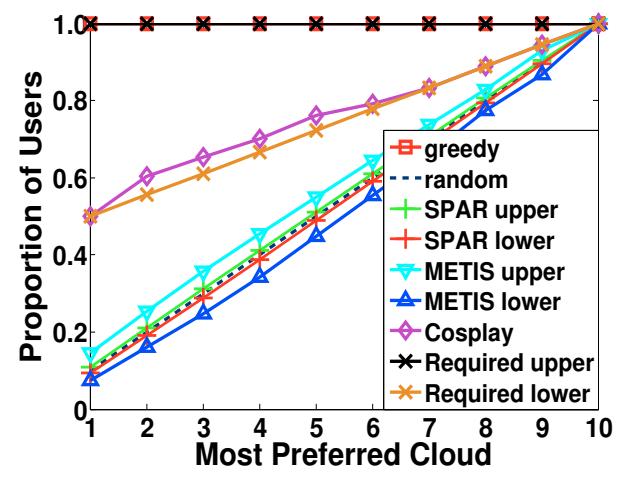

Figure 3.14: QoS comparison

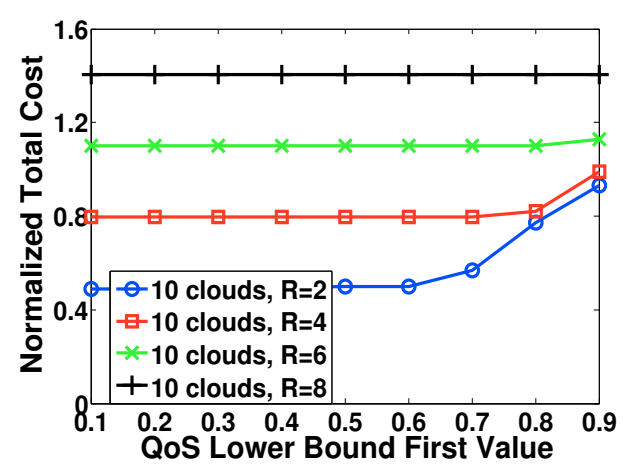

Figure 3.13: Cost of cosplay (IV)

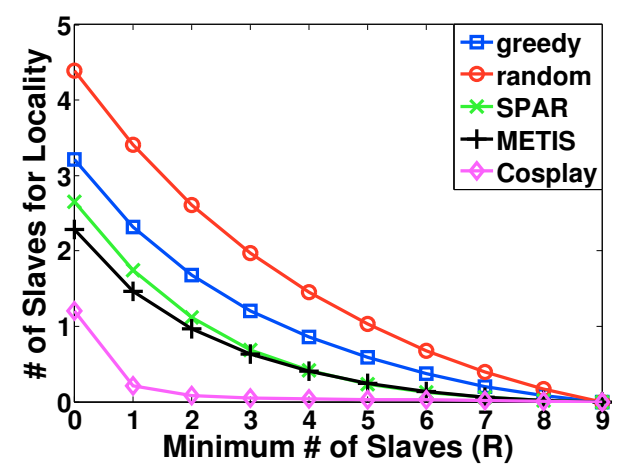

Figure 3.15: Slave numbers

are not obviously influenced by the number of most preferred clouds. In contrast, when every user only considers using her 2 most preferred clouds to host her master, cosplay achieves much less cost reduction. This phenomenon implies that 2 most preferred clouds of each user do not seem to cover the social relations among users, while 4 most preferred clouds of each user can have clouds of friends overlapped and masters of friends co-located, and thus fewer slaves are needed for social locality and the total cost can be much less than the 2-clouds case. However, this advantage is gradually compensated as the minimum number of slaves required by every user increases.

Fig. 3.12 shows the total costs cosplay achieves when the QoS requirement varies with $R=0$. As the first value of $\vec{Q}_{l}$ increases, the number of users that are allowed to be role-swapped decreases and thus less room is left for optimization. When the first value is small, it does not affect the amount of cost that can be saved. Although more users are allowed to be role-swapped, the number of roleswaps that can lead to actual cost reduction is limited - allowing more users to be role-swapped does not necessarily indicate more cost reduction. When the first value becomes large enough, operating on fewer most preferred clouds achieves more cost reduction, which aligns with our intuition that placing together a small number of users instead of straddling them across clouds could save the cost. As 
cosplay operates on a larger number of most preferred clouds, it is easier for the cost reduction to be affected by the first value as this value grows. This is natural as more clouds available means more role-swaps can be done to save cost. Hence, it is easier to be affected when the number of permitted users decreases.

Fig. 3.13 provides the total costs cosplay achieves as both the QoS requirement and $R$ vary when operating on all the 10 most preferred clouds. The case with a larger $R$ tends to be less affected by $\vec{Q}_{l}$ than the case with a smaller $R$. This is because when $R$ is larger, the room for optimization becomes small due to that the number of role-swaps that leads to cost reduction becomes small. It can be small enough that even when $R=8$ the QoS requirement does not have any effect on the cost. With any given QoS requirement, a larger $R$ always comes with a lower cost reduction, consistent with Fig. 3.11.

Fig. 3.14 visualizes the QoS vectors of the placements produced by all the methods over the 10 clouds. No doubt that greedy has the best QoS. Random has the linear QoS as expected. SPAR or METIS partitions a graph into a given number of partitions. With 10 clouds, there exist $10 !=3,628,800$ different ways of placing the 10 partitions upon the 10 clouds. Each placement has its own QoS. Out of all 10! QoS vectors, we obtain the largest value and the smallest value for each of the 10 dimensions of the QoS vector. We can therefore draw the upper and lower QoS bounds for SPAR and METIS, respectively. SPAR and METIS are only able to produce QoS similar to random, while cosplay can always keep the QoS within any pre-defined upper and lower bounds.

Fig. 3.15 investigates how many more slaves we need to ensure the social locality for every user, except the minimum number of slaves that are maintained for data availability (some of them may also serve social locality). This figure draws the average number of additional slaves needed for social locality in the placements produced by different methods. We see that, while meeting the data availability requirement, cosplay always needs the fewest number of additional slaves for social locality of all users. Cosplay not only minimizes the cost, it also reduces the number of replicas. What is interesting is that, SPAR, an algorithm of minimizing the replica number, is beat by METIS. This is because SPAR runs as a procedure of responding to a series of edge creation events and only guarantees the minimal number of replicas of involved users in a local sense, while METIS takes the whole social graph as input and thus achieves better results in a global sense.

\subsubsection{Results on Continuous Cost Reduction}

We note that, as stated in Section 3.5.1, we mainly focus on the time periods after the 16th month. The two peaks of user population growth in the 26th and 37 th month are also reflected in our results. 


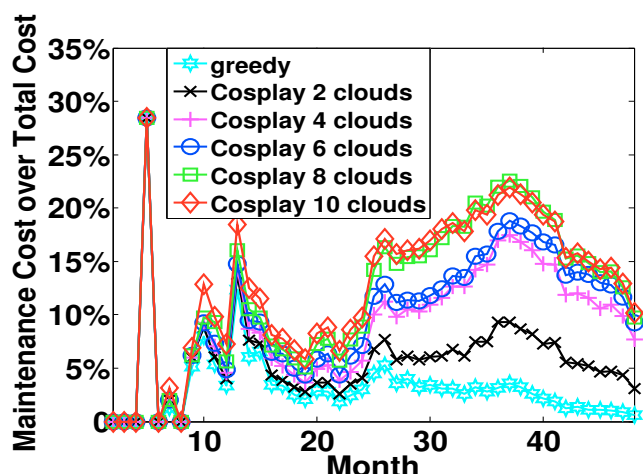

Figure 3.16: Maintenance cost

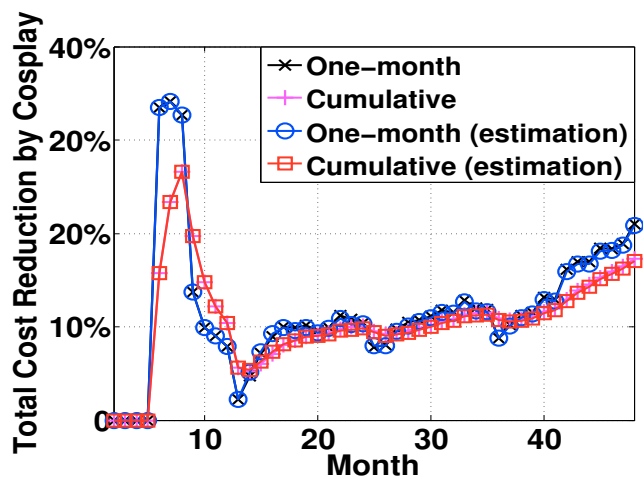

Figure 3.18: 2 clouds

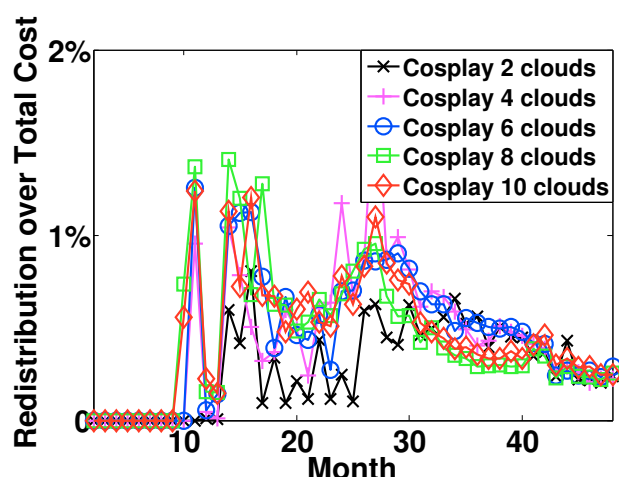

Figure 3.17: Redistribution cost

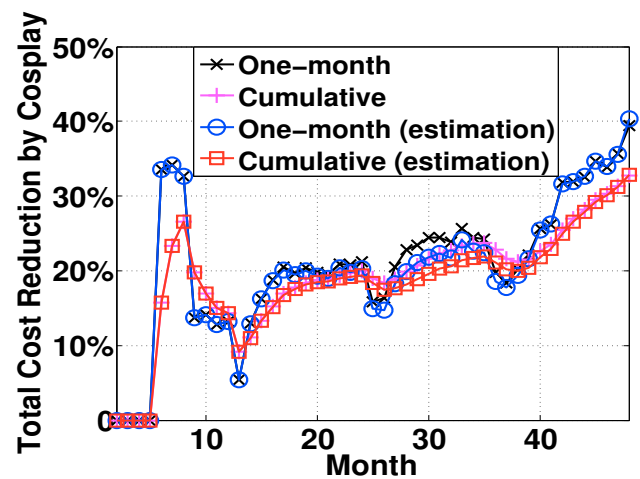

Figure 3.19: 4 clouds

Fig. 3.16 and 3.17 report the ratios of the maintenance cost and the redistribution cost over the total cost in each month, respectively. Fig. 3.16 verifies our cost model as the maintenance cost of greedy occupies less than $5 \%$ of the total cost, which is the reason why we can neglect the maintenance cost incurred by newly-joined users in our approximated cost model. Cosplay significantly reduces the total cost for each month, causing the maintenance cost to occupy larger proportions out of the total cost. Fig. 3.17 shows that the redistribution cost always keeps below $2 \%$ of the total cost of a month.

From Fig. 3.18 to Fig. 3.22, we find the one-time cost reduction for each month and the cumulative cost reduction until each month, compared with greedy. We make several observations. Firstly, our estimation approach performs effectively. The one-month and the cumulative cost reductions achieved by running cosplay on estimated costs do not deviate too much from, and almost overlap with reductions achieved by running cosplay on real-world costs. Secondly, the cost reduction climbs up as time elapses, and increases as more clouds are involved for each user. In the 10-clouds case, the accumulative total cost reduction goes towards more than $40 \%$. Thirdly, the cost reduction can be deteriorated by large monthly growth rates, as in the months where local peaks occur. However, as discussed previously, the real-world monthly growth 


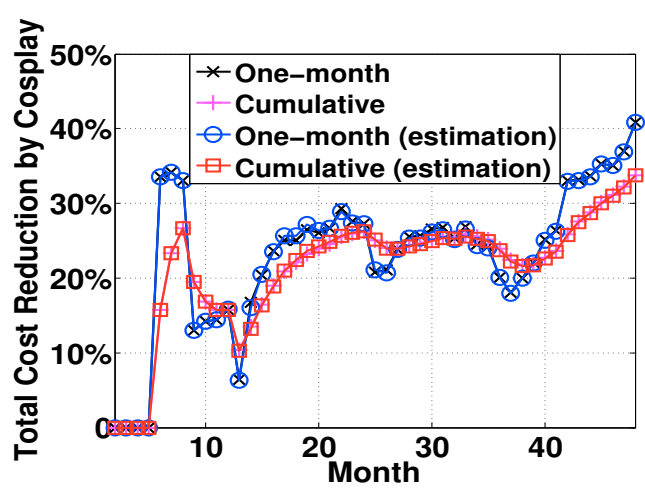

Figure 3.20: 6 clouds

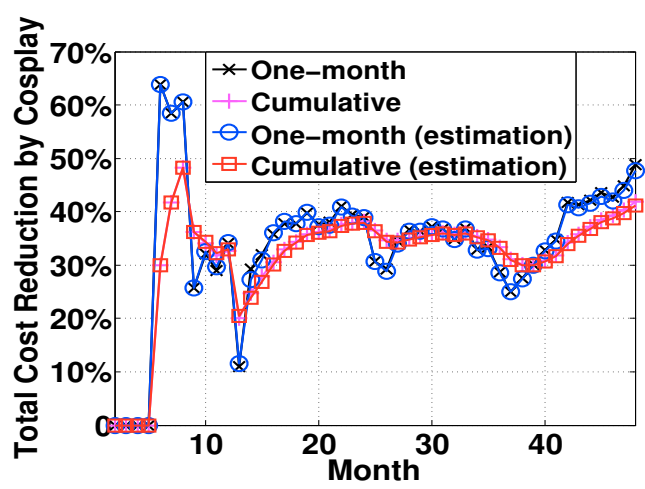

Figure 3.22: 10 clouds

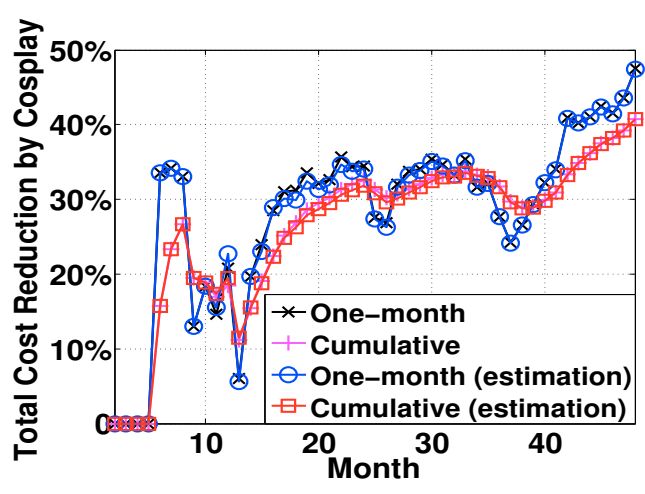

Figure 3.21: 8 clouds

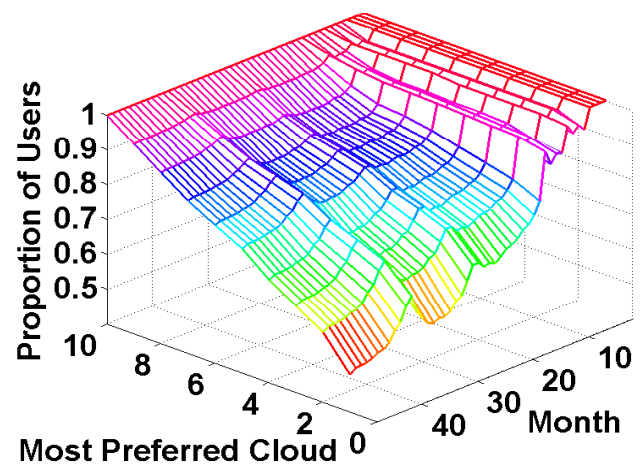

Figure 3.23: QoS variation

rate is usually quite small and thus we can expect significant cost reductions. OSN provider can also determine whether to apply cost optimization to a billing period based on an estimation of the OSN growth rate.

Using Fig. 3.22 as an example, we report that out of all 48 months, for traffic cost, 4 months have the users' average prediction error of less than $\pm 1 \times$ and 39 months have the users' average prediction error of less than $\pm 5 \times$; for storage cost, 35 months have the users' average prediction error of less than $\pm 1 \%$ and 45 months have the users' average prediction error of less than $\pm 2 \%$. The small prediction error in storage cost tends to be the cause of the small deviation of the cost reduction calculated based on predicted cost. This is natural because the reduction of storage cost dominates the total cost reduction, and a user's stored data accumulates and becomes much bigger than the amount of her traffic amount as time elapses.

Fig. 3.23 visualizes in the 10 -clouds case how the QoS vector could vary during the 48 months when running cosplay. New users joining the service in each month are greedily placed, causing the QoS curves to fluctuate from one month to another. Adjusting replica locations at the beginning of each month also influences the QoS curves. 


\subsection{Discussions}

Having demonstrated the evaluation results of our algorithm, now we selectively discuss some related issues.

\subsubsection{Algorithm Complexity}

Cosplay has a time complexity of $O\left(N^{2} \delta\right)$, where $N$ is the number of clouds in the system and $\delta$ is the number of iterations executed, given that real-world OSN services often enforce a constant limit of the number of friends a user can have; without such a limit, in the worst case where every user was a friend of every other user, the complexity would be $O(|V| \delta)$ where $|V| \gg N$ is the number of users in the system. As an example, let's consider one iteration in the single role-swaps procedure, assuming each user has up to $C$ friends. The first step of the random selection of a user is $O(1)$. In the second step, checking the feasibility of a single role-swap by Algorithm 1 takes $O(N)$ and thus checking all single role-swaps of a user takes $O\left(N^{2}\right)$. Then, calculating the cost reduction of a single role-swap by Algorithm 3 takes $O(C)$ and thus calculating the cost reductions of a user's all feasible role-swaps takes $O(N C)$. The third step picks up the role-swap with the largest cost reduction with a complexity of $O(N)$. The complexity of one iteration is thus $O(1)+O\left(N^{2}\right)+O(N C)+O(N)=O\left(N^{2}\right)$, and for $\delta$ iterations it is $O\left(N^{2} \delta\right)$. In our evaluations, we pre-specify that each user, on average, is allowed 100 times of being selected for role-swaps, i.e., $\delta=100|V|$; however, through all evaluations with various settings, our algorithm runs at most a few more than $10|V|$ iterations before no role-swap can be done to reduce the cost.

\subsubsection{Optimality Gap}

Although cosplay only finds a local optimal solution to our cost optimization problem, it performs empirically much better than other placement approaches in Section 3.5.3. Figuring out the optimality gap is challenging as finding the global optimal solution is NP-hard. Nevertheless, using the small-scale example in Fig. 3.1, we can have a rough sense about how much the optimality gap would be. We have 11 users and 3 clouds, so there are $3^{11}=177147$ possible placements of masters; slaves are placed to ensure social locality and data availability. We consider the cases of $R=0$, where slave replicas only serve the purpose of ensuring every user's social locality, and $R=1$, where every user has at least 1 slave replica no matter it is for social locality or data availability. For a given QoS requirement and a given data availability requirement, we can obtain the placement with the minimal (optimal) cost and the placement with the maximum (worst) cost by enumerating all the feasible solutions; we also run 


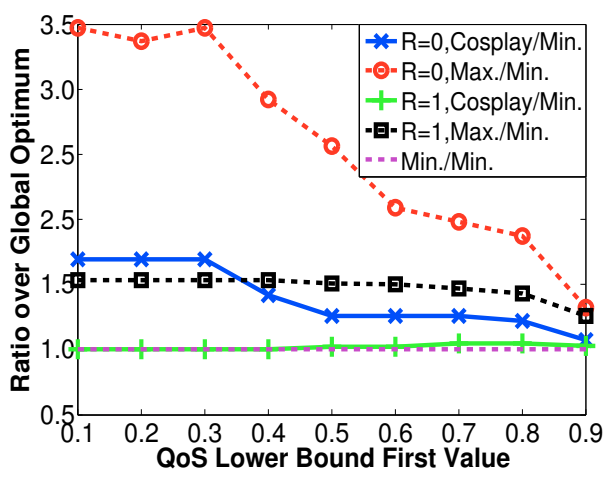

Figure 3.24: Optimality gap

cosplay. Fig. 3.24 is the result. For example, when $R=0$ and the QoS first value is 0.5 , cosplay finds the solution whose cost is 1.26 times of the optimal cost while in this case the maximum cost is 2.56 times of the optimal cost; when $R=1$, cosplay almost always finds an optimal solution. Compared with the former, there is less room for optimization in the latter case; one may imagine that in the extreme case of $R=2$ no optimization can be done, as the total cost is fixed no matter how masters and slaves are placed. We deem that an approximation ratio like this is reasonably good.

\subsubsection{Design Alternatives}

It appears that role-swaps limit the solution to the initial placement, i.e., a role swap does not seem to be able to move a user's data to a cloud that has none of her replicas in the initial placement. We will demonstrate and explain in the following that (1) allowing movements does not help much in reducing the cost, and (2) only role-swaps alone can move a considerable amount of users to the clouds that do not host their replicas in the initial placement. Although not included in this chapter, Algorithm 3 and 5 have already been adapted to allowing movements. A movement refers to moving a master replica from one cloud to another cloud which does not have a slave replica of the same user. As in role-swaps, social locality also needs to be ensured by creating slaves of neighbors if necessary. After adaption to movements, in each iteration, our algorithm can perform the operation, either a role-swap or a movement, whose cost reduction is the maximal out of all feasible role-swaps and movements. We thus run additional evaluations as in Fig. 3.25 and 3.26. Fig. 3.25 indicates that even allowing movements, the number of movements performed only occupies a small portion of the total number of all the operations performed; in fact, the placement obtained has almost the same cost as in the only role-swaps case (which is not shown in the figure). The reason that role-swaps tend to suffice and movements may not be important is that a movement can hardly reduce the 


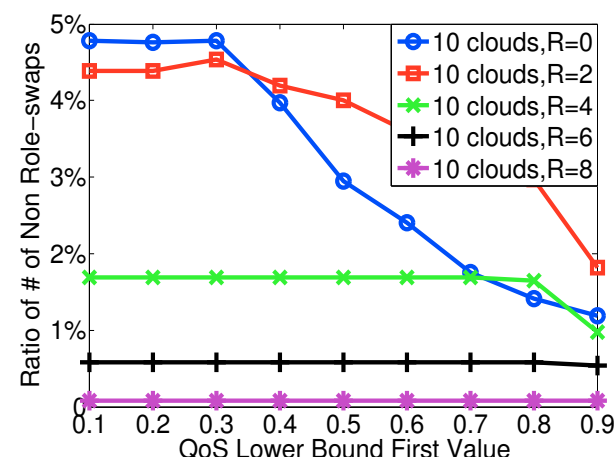

Figure 3.25: Ratio of \# of movements

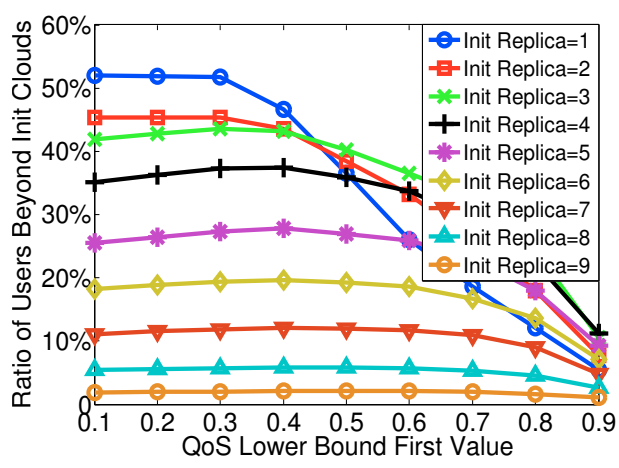

Figure 3.26: Users beyond initial locations

total cost, unlike a role-swap: firstly, a movement incurs redistribution cost itself by moving the master replica to the destination cloud, while a role-swap does not have this cost; secondly, it may also incur redistribution cost by creating slaves of neighbors at the destination cloud. In a role-swap of a user, as the user has a slave serving social locality at the destination cloud, it is highly likely that neighbors already have masters there, which does not hold for a movement. Fig. 3.26 indicates that, when no movements allowed and only role-swaps performed, a considerable portion of users have ever had their masters swapped to clouds other than where their slaves are placed in the initial placement. For example, when the QoS first value is 0.1 , for users who have 2 replicas (including master) in the initial placement, $45 \%$ of them have ever been beyond the initial clouds while cosplay runs. In this figure we set $R=0$. As a user's slaves are created to maintain the social locality of a neighbor's master, the new slaves will cause the user's master to be swapped to the clouds where they are created; a user's master is not restricted to those clouds hosting her slaves in the initial placement.

\subsubsection{Requirement Variation}

As the OSN evolves, the OSN provider's data availability requirement and QoS requirement may change. The change of the former, however, can be easily handled. If a user needs more slaves to improve the data availability, one can choose some clouds and create the needed slaves there. Note that such slaves only accept propagated writes for consistency, as social locality is already ensured by existing slaves. The cost associated with these new slaves created for data availability does not reply on at which clouds they are placed. On the other hand, if fewer slaves are expected as the current level of data availability is unnecessarily high, one can then remove slaves which do not serve the social locality. The QoS requirement can also be changed by the OSN provider so that an existing placement that satisfies the old requirement may not necessarily meet the new requirement; also, when users move their locations and their preferences for 
clouds change accordingly, the QoS of a placement may vary and not satisfy the QoS requirement any more. So in this case, how to minimize its cost while making such a placement satisfy the new QoS requirement and the data availability requirement? One approach can be firstly moving data to make the placement meet the QoS requirement, which is always feasible, and afterwards running our algorithm as we do to minimize its cost. Here one may want to achieve the first step at the minimum redistribution cost by composing some algorithm based on our adapted version of cosplay.

\subsection{Summary}

In this chapter, we study the problem of optimizing the monetary cost spent on cloud resources when deploying an online social network (OSN) service over multiple geo-distributed clouds. We model the cost of OSN data placement, quantify the OSN quality of service (QoS) with our vector approach, and address OSN data availability by ensuring a minimum number of replicas for each user. Based on these models, we present the optimization problem of minimizing the total cost while ensuring the QoS and the data availability as required. We propose cosplay as our algorithm. By extensive evaluations with large-scale geosocial Twitter data, cosplay is verified to incur substantial cost reductions over existing and state-of-the-art approaches. It is also characterized by significant one-time and accumulated cost reductions over 48 months with the QoS and the data availability always meeting pre-defined requirements. 



\section{Chapter 4}

\section{OSN Data Placement across Clouds with Multiple Objectives}

\subsection{Introduction}

Cloud-based Internet services intrinsically have multiple system objectives, including budgeting the monetary expenditure spent on cloud resource usage [44, 92], ensuring the service quality perceived by users (e.g., access latency) [61, 95], and even reducing the carbon footprint of the service [40,58], to name a few. Such services are often deployed over multiple geographically distributed clouds to meet the demand of users at diverse regions and for fault tolerance, which, in turn, provides a unique opportunity to optimize the service for many of its system objectives by carefully choosing at which cloud to place the data accessed by users. For example, different clouds may charge different prices for the same amount of resource consumption, have different proximity to users, and emit different amounts of carbon for the same workload, i.e., they have different carbon intensities. Data placement determines how the workload of a service is distributed over clouds, and thus affects various system objectives of the service.

The data placement problem is particularly challenging for multi-cloud services that are socially aware, where users build social relationships and share contents with one another, as reflected by Online Social Network (OSN) services and many non-OSN services with social components [2]. Fig. 4.1 illustrates how one such service is provided at distributed clouds to serve users at different locations, where every user can access every cloud and users form an OSN via online friendships. The challenges for optimizing data placement for such services mainly manifest themselves as follows:

First, because of social relations and interactions of users in a socially aware service, the data of every user are interconnected with data of some others, and data placement on the basis of individual users probably cannot yield optimal results. With heavy interactions between online friends [48, 90], for example, it would be better to have their data placed closely. Ideally, if the data of a user and the data of her friends are always co-located at the same cloud, the user 


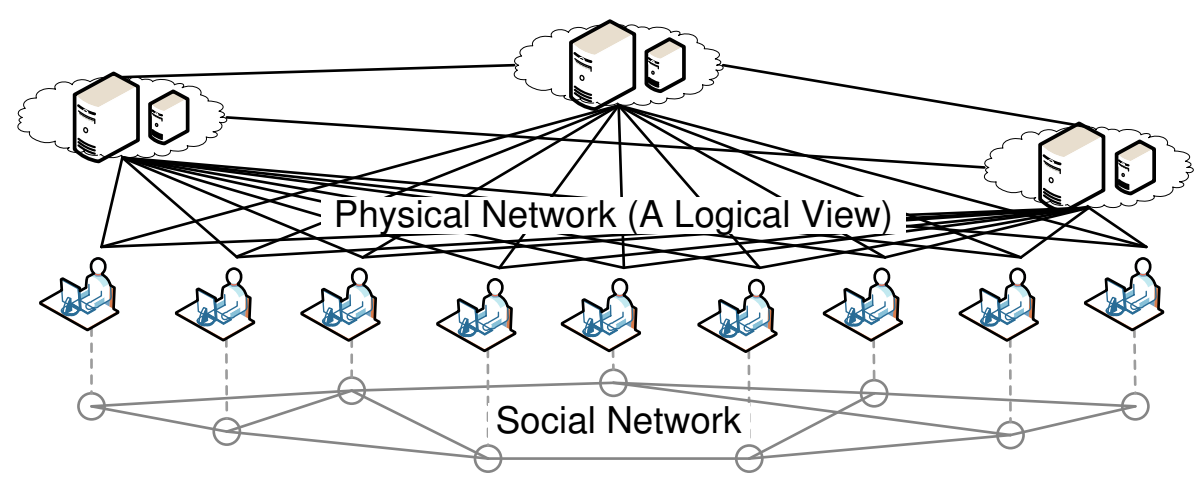

Figure 4.1: A multi-cloud socially aware service

can access her friends' data without going to another cloud, and thus save the additional delay and traffic associated with further operations. This feature distinguishes social data placement from content placement in conventional content distribution networks, where data are independently delivered to users.

Moreover, the diverse objectives for data placement are often intertwined and even contradictory, and may not be satisfied simultaneously [40]. The social nature, for example, requires friends' data to be placed closely. For low access latency, data accessed by each user - including those of her friends and her ownare preferred to be located at the clouds close to a user. However, to reduce the monetary expense or the carbon footprint, it is beneficial to place more users' data at clouds that are cheaper in price or more efficient in carbon intensity. A data placement approach thus needs to be capable of seeking trade-offs among multiple objectives.

Further complicating the problem are the multi-cloud master-slave paradig$\mathrm{m}$ [80] and the multi-cloud access policies [82]. When the data of every user have a master replica and multiple slave replicas, as is often the case in many services, these replicas contribute differently to system objectives. The location of a user's master contributes to the write latency perceived by all those who write to this user's data. The locations of a user's slaves contribute to the read latency, and different users may read different replicas of a user. If a data replica is not available at a current cloud, different multi-cloud access policies regulate differently "where" and "how" to obtain the required data from another cloud, thus potentially leading to different optimal data placements.

Unfortunately, most previous optimization research on multi-cloud or multidata-center services $[40,58,61,74,76,95]$ cannot capture users' social relations and their interactions in socially aware services. There are multiple studies on multi-cloud OSN and social media services [60, 86, 92], but they do not address the carbon issue and are not able to weigh costs of multiple dimensions. Furthermore, except the work in [60], little has been done to investigate multicloud data placement in the context of the master-slave paradigm - which is very 
common in reality — and multi-cloud access policies.

In this chapter, we investigate and solve the multi-objective data placement problem of multi-cloud socially aware services using a combination of modeling, analysis, and extensive simulations. We capture this problem by building a model framework that generalizes to a large variety of system objectives. We address the aforementioned challenges by proposing a novel approach that leverages the graph cuts technique $[25,26,56]$. We verify our models and approach via evaluations driven by large-scale real-world data.

Starting with modeling the carbon footprint, the service quality, the intercloud traffic of the socially aware service, as well as the reconfiguration cost incurred by changing a given data placement to another, we generalize our models to cover a wide range of system objectives of different dimensions, allowing them to be treated within a common framework. The data placement problem is thus about finding best locations for each user's master and slave replicas in order to minimize the total cost. An interesting observation of our models is that the cost of every dimension of a socially aware service can be naturally cast into one or both of the two parts: the unary cost that depends on the locations of replicas of an individual user, and the pairwise cost that depends on the locations of replicas of a pair of users, i.e., one user who conducts read and write operations and the other user whose data are read or written.

Our core contribution is a novel approach that solves the data placement problem. Intuitively, the unary cost and the pairwise cost correspond to vertices and edges of a graph respectively, motivating us to connect our data placement problem that is centered around cost minimization with the problem of finding the minimum cut of a graph, and to solve the former via solving the latter with the help of the graph cuts technique. Towards this end, we propose to decompose our original problem into two subproblems and solve them alternately in multiple rounds. In one subproblem, given the locations of all slaves, we identify the optimal locations of all masters by iteratively cutting the corresponding graphs. In the other subproblem, we place all slaves given the locations of all masters, where we find that the optimal locations of each user's slaves are independent and a greedy scheme that takes account of all objectives can usually be sufficient. Our approach achieves good data placements overall. On one hand, to the best of our knowledge, the state-of-the-art graph-cut technique guarantees the best solutions; on the other hand, our greedy scheme can empirically achieve results close to the theoretical optimum. By applying it separately to each community of the entire user base, our approach further scales to a huge user population. Doing so may degrade the optimization performance, but only moderately due to the community structure of users in socially aware services.

With 107,734 users interacting over 2,744,006 social relations that span all across the US, we perform data placement over 10 distributed clouds. Our eval- 
uations demonstrate the following results: (1) While there is no other approach known to us that optimizes the multi-objective data placement for multi-cloud socially aware services, our approach significantly outperforms several standard and de facto practices, such as random and greedy placement, in all objective dimensions including carbon, distance and traffic in a variety of settings; (2) Our model can be easily tuned to achieve different trade-offs among multiple objectives, and to help decide whether a specific optimization outcome is worth the effort of conducting this optimization; (3) Our approach converges fast, e.g., the first 3 iterations reach approximately $97 \%$ of the total cost reduction that can be achieved; (4) Our approach scales, e.g., by partitioning the user base into 4 communities and applying our approach independently to each community, we obtain a speedup of 4.5 in execution time with the optimization performance degraded only slightly by $6 \%$; (5) While using graph cuts to place masters, our approach is more affected by the initial placement of all replicas than the approach we use to place slaves, and our choice of greedy placement is appropriate and efficient; (6) How good the optimization results are does not correlate with the number of iterations executed.

The rest of this chapter proceeds as follows. Section 4.2 presents our models and generalizations. Section 4.3 formulates the problem. We propose our algorithmic approach in Section 4.4. We demonstrate and interpret our experimental data, settings and results in Section 4.5. We discuss the regularity condition, and the optimality, scalability of our algorithm in Section 4.6. We conclude this chapter in Section 4.7.

\subsection{Models}

We introduce the system settings of the multi-cloud socially aware service. We model its carbon footprint, operation distance, inter-cloud traffic, and reconfiguration cost. We generalize these models to cover a wider range of system objectives, based on which we formulate the problem of determining the best data placement with the optimal objectives.

\subsubsection{System Settings}

We target a multi-cloud socially aware service, where every user can access every cloud, every cloud can access every other cloud, and users form an OSN via online friendships. Each cloud is located at a different geographic region and each user has replicas of her data stored in the clouds. We consider the single-mastermulti-slave paradigm [20,80], where every user has one replica as a master and multiple other replicas as slaves. Each replica is stored in one cloud. The cloud that hosts a user's master replica is the user's master cloud, and those that host 
a user's slave replicas are the user's slave clouds. Central to user interactions are the read and write operations between users. We focus on the number of reads and writes.

A service with data partitioned and replicated across clouds often follows some multi-cloud access policies about "where" and "how" to obtain the required data from a remote cloud if they are unavailable at a local cloud. This happens when, e.g., a user accesses the service via the web and her read request is directed by DNS to a cloud, but the data requested turns out to be at a different cloud. We handle such access policies in this chapter as follows. "Where" is captured in our model by the function $z_{u, v}$, which selects a cloud out of user $v$ 's master and slave clouds by a given policy in order to serve user $u$ 's requests that access $v$ 's data. Note that $z_{u, v}$ only applies to read operations, since write operations are always executed in master clouds, with propagations to slaves afterwards. "How" is captured as either a relay mode or a redirect mode [82]. The former means the local cloud reads the data from another cloud and then returns the data to the user. The latter means the local cloud redirects the user to another cloud and lets the user retrieve the data on her own.

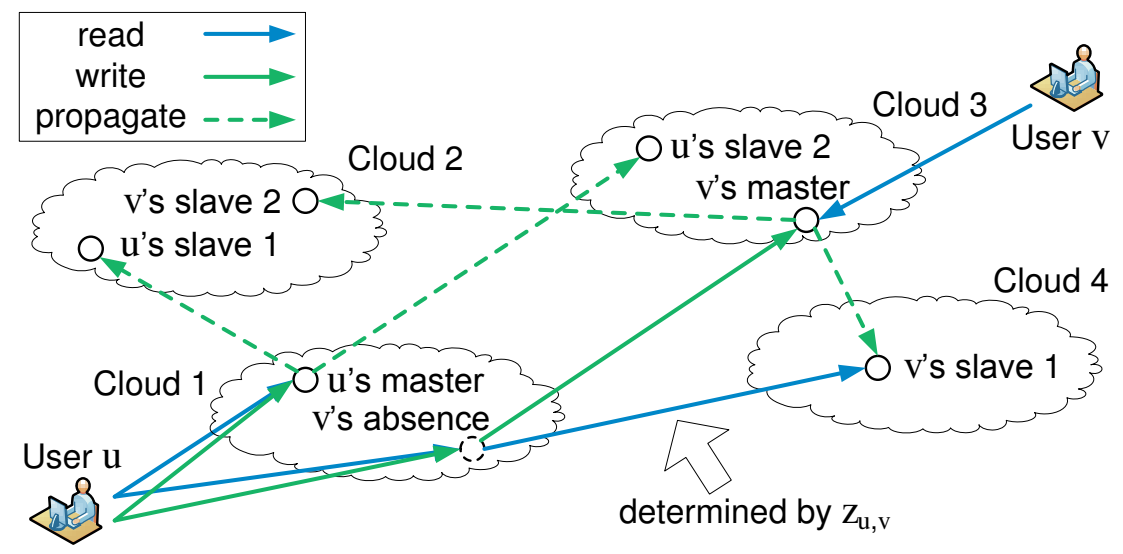

Figure 4.2: Reads and writes over multiple clouds

Before we build analytical models, Fig. 4.2 illustrates the general idea about how a multi-cloud socially aware service serves its users in the relay mode, and how multiple system objectives such as the carbon footprint, the operation distance (a concept that we define as a metric of QoS), and the inter-cloud traffic are calculated given the data placement. There are 2 users $u, v$ and 4 clouds, where each user has a single master replica and 2 slave replicas placed as shown in this figure. When signing in to the service, $u$ and $v$ are connected to their master clouds 1 and 3, respectively, and are served by these two clouds thereafter.

Let us look at the read operations firstly. $u$ reads her own data at cloud 1 and $v$ reads her own data at cloud 3. $u$ also reads $v$ 's data; however, due to the absence of $v$ 's data at cloud 1 , cloud 4 is selected by the access policy $z_{u, v}$ 
and is contacted by cloud 1 to fetch $v$ 's data. Then such data are relayed to $u$ via cloud 1 . Next, let us look at the write operations. $u$ writes her own data at cloud 1 , and such write is propagated to $u$ 's slaves at cloud 2 and 3 . $u$ writes $v$ 's data, and due to the absence of $v$ 's master at cloud 1 , such write is forwarded to cloud 3. Then the write to $v$ at cloud 3 also propagates to $v$ 's slaves at cloud 2 and 4 . Finally, let us see how system objectives are calculated:

- Carbon footprint: $u$ reads and writes her own data at cloud 1 , reads $v$ 's data at cloud 4 , and writes $v$ 's data at cloud 3, so the carbon footprint incurred by these operations is generated at clouds 1,3 and 4, respectively. Similarly, the carbon footprint incurred by $v$ 's read is generated at cloud 3 ; the carbon footprint incurred by all propagated writes is at clouds 2,3 and 4 .

- Operation distance: $u$ 's read and write on her own data travels the distance between $u$ and cloud 1. $u$ 's read on $v$ 's data travels the distance between $u$ and cloud 1 , plus that between cloud 1 and cloud 4 ; $u$ 's write on $v$ 's data travels the distance between $u$ and cloud 1 , plus that between cloud 1 and cloud 3. $v$ 's read on her own data travels the distance between $v$ and cloud 3 .

- Inter-cloud traffic: The traffic between clouds includes that between cloud 1 and cloud 4 due to $u$ 's read, that between cloud 1 and cloud 3 due to $u$ 's write, as well as all those incurred by propagated writes.

Fig. 4.2 exemplifies the relay mode; in the redirect mode, the operations distance does not involve any distance between clouds. Besides, the carbon intensity, i.e., the amount of carbon emitted for processing one unit of workload, may be different at different clouds. Our models capture more details, including the reconfiguration cost, i.e., the cost of changing one data placement to another.

We introduce the notations that are used in the rest of this chapter. We use $u, v$ to denote users and $i, j$ to denote clouds. The decision variables to be solved are $\mathbf{m}_{u}$ and $\mathbf{s}_{u, l}, l=1, \ldots, \mathrm{k}, \forall u$, indicating the location of $u$ 's master (i.e., the ID of $u$ 's master cloud) and those of $u$ 's k slaves (i.e., the IDs of $u$ 's $\mathrm{k}$ slave clouds), respectively. $\mathrm{k}$ is the number of slave replicas each user has. $r_{u}$ and $w_{u}$ denote the number of reads and that of writes conducted by user $u$ on her own data. $\mathrm{r}_{u v}$ and $\mathrm{w}_{u v}$ denote the number of reads and that of writes conducted by user $u$ on user $v$ 's data. $\mathbb{N}_{u}$ is the set of user $u$ 's neighbors, where two users are considered neighbors if and only if there exists at least one read or write operation between them. $z_{u, v}\left(\mathbf{m}_{u}, \mathbf{m}_{v}, \mathbf{s}_{v, 1}, \ldots, \mathbf{s}_{v, \mathrm{k}}\right)$ is the function returning the ID of the selected cloud according to a given policy. $\delta(x, y)$ is a binary function that returns 1 if $x \neq y$, and 0 otherwise. Other notations that are not introduced here are explained just before they are used. 


\subsubsection{Modeling Carbon Footprint}

The total carbon footprint of the service depends on the workload of each cloud, i.e., the read and write operations which are executed at each cloud, and also on the carbon intensity of the region where a cloud is located. A user reads and writes her own data at her master cloud, and all of a user's writes are eventually propagated from her master cloud to all her slave clouds for consistency. When a user $u$ reads another user $v$ 's data, the cloud determined by $z_{u, v}$ serves such reads. When $u$ writes $v$ 's data, the writes go to $v$ 's master cloud for execution, and further propagate to all of $v$ 's slave clouds. ${ }^{1}$

Let $\varepsilon$ be the energy consumption of a single read or write operation and $e_{i}$ be the carbon intensity of the region where cloud $i$ is located. We write the total carbon footprint as

$$
\sum_{u} D_{u}^{c}+\sum_{u} \sum_{v \in \mathbb{N}_{u}} V_{u \rightarrow v}^{c}
$$

where $D_{u}^{c}=D_{u}^{c \prime}+D_{u}^{c \prime \prime}$, with $D_{u}^{c \prime}=\varepsilon \mathrm{e}_{\mathbf{m}_{u}}\left(\mathrm{r}_{u}+\mathrm{w}_{u}+\sum_{v \in \mathbb{N}_{u}} \mathrm{w}_{v u}\right)$, representing the carbon at $u$ 's master cloud incurred by all of $u$ 's reads and writes on her own data and all the writes conducted by $u$ 's neighbors on $u$ 's data, and $D_{u}^{c \prime \prime}=$ $\sum_{l=1}^{k}\left(\varepsilon \mathbf{e}_{\mathbf{s}_{u, l}}\left(\mathrm{w}_{u}+\sum_{v \in \mathbb{N}_{u}} \mathrm{w}_{v u}\right)\right)$, representing the carbon at all of $u$ 's slave clouds incurred when all writes to $u$ are propagated to her slaves, and $V_{u \rightarrow v}^{c}=\varepsilon \mathrm{e}_{z_{u, v}} \mathrm{r}_{u v}$ refers to the carbon at the cloud $z_{u, v}$ when $u$ reads her neighbor $v$.

Our model here applies to both the relay mode and the redirect mode, as the carbon footprint focuses on at which clouds the read and write operations occur and it does not care about how to reach such clouds.

\subsubsection{Modeling Operation Distance}

We define the operation distance of a service as the total geographic distance traveled by all reads and writes occurred in this service, and we use this notion as a measure of service quality.

In the relay mode, for example, if a user issues a read request and this request is forwarded due to data absence at her master cloud, then the distance traveled by this read is the distance between the user and her master cloud plus the distance between her master cloud and the cloud that this request is forwarded to. ${ }^{2}$ All operations issued by a user firstly go to her master cloud, and then if the data required by some operations are not available there, such operations continue to travel to the destination clouds which have the required data. Thus,

\footnotetext{
${ }^{1}$ Issues out of the scope of our work include write conflicts resolution [80]. We assume such issues are addressed by existing techniques, which does not affect our work as long as all writes are eventually executed.

${ }^{2}$ Aligning with eventual consistency, we assume a write operation returns to the user as soon as it is completed on the maser replica [20]. Therefore, the operation distance of a write does not involve propagations.
} 
the operation distance is calculated as follows, where $\mathrm{d}_{\text {.,. }}$ is the distance between a user and a cloud, or between two clouds:

$$
\sum_{u} D_{u}^{d}+\sum_{u} \sum_{v \in \mathbb{N}_{u}} V_{u \rightarrow v}^{d}
$$

where $D_{u}^{d}=\mathrm{d}_{u, \mathbf{m}_{u}}\left(\mathrm{r}_{u}+\mathrm{w}_{u}+\sum_{v \in \mathbb{N}_{u}}\left(\mathrm{r}_{u v}+\mathrm{w}_{u v}\right)\right)$ is the user-cloud distance, i.e., the total distance traveled by all of $u$ 's operations to go from $u$ herself to her master cloud, and $V_{u \rightarrow v}^{d}=\mathrm{d}_{\mathbf{m}_{u}, z_{u, v}} \delta\left(\mathbf{m}_{u}, z_{u, v}\right) \mathbf{r}_{u v}+\mathrm{d}_{\mathbf{m}_{u}, \mathbf{m}_{v}} \delta\left(\mathbf{m}_{u}, \mathbf{m}_{v}\right) \mathbf{w}_{u v}$ is the inter-cloud distance, i.e., the sum of the total distance traveled from $u$ 's master cloud to the cloud $z_{u, v}$ when $u$ reads $v$ and the total distance traveled from $u$ 's master cloud to $v$ 's master cloud when $u$ writes $v$.

In the redirect mode, a user issues a read request to her master cloud which redirects this request to a proper cloud, i.e., the user issues the same read request to this proper cloud to get the required data on her own. We may consider two cases. In the first case where every read request needs a redirection, the operation distance of a read is the distance between a user and her master cloud plus the distance between the user and the cloud that she contacts to retrieve the required data. In the second case where the user may have a local cache, e.g., a web cookie, so that she needs to contact her master cloud only once to cache the redirect information before reading the data of others. For all reads to others, there is a common distance between the user and her master cloud; for each later read, the distance is only that between the user and the cloud that she contacts to retrieve the required data. Based on $D_{u}^{d}$ in (4.2), the operation distance for these two cases are calculated as follows, respectively:

$$
\sum_{u} D_{u}^{d}+\sum_{u} \sum_{v \in \mathbb{N}_{u}} V_{u \rightarrow v}^{d}{ }^{\prime}
$$

and

$$
\sum_{u} D_{u}^{d^{\prime}}+\sum_{u} \sum_{v \in \mathbb{N}_{u}} V_{u \rightarrow v}^{d}{ }^{\prime}
$$

where $D_{u}^{d^{\prime}}=\mathrm{d}_{u, \mathbf{m}_{u}}\left(\mathrm{r}_{u}+\mathrm{w}_{u}+1\right), V_{u \rightarrow v}^{d}{ }^{\prime}=\mathrm{d}_{u, z_{u, v}} \mathrm{r}_{u v}+\mathrm{d}_{u, \mathbf{m}_{v}} \mathrm{w}_{u v}$. Note that there is no inter-cloud distance involved in the redirect mode.

\subsubsection{Modeling Inter-Cloud Traffic}

In the relay mode, the inter-cloud traffic can be incurred by inter-cloud operations, e.g., reads and writes that cannot be completed at a local cloud due to absence of data and are thus executed at a remote cloud. The total amount of such inter-cloud traffic is follows, assuming $t$ bytes of traffic incurred by a single operation:

$$
\sum_{u} \sum_{v \in \mathbb{N}_{u}} V_{u \rightarrow v}^{t}
$$

where $V_{u \rightarrow v}^{t}=\mathrm{t}\left(\delta\left(\mathbf{m}_{u}, z_{u, v}\right) \mathbf{r}_{u v}+\delta\left(\mathbf{m}_{u}, \mathbf{m}_{v}\right) \mathrm{w}_{u v}\right)$ is the sum of the inter-cloud traffic incurred when $u$ reads and writes $v$. 
Note that the inter-cloud traffic incurred by propagated writes for replica consistency, however, is ruled out of our optimization framework. Such traffic is fixed when the inputs are given. It only depends on the number of slave replicas of each user and the number of writes received by each user; it does not vary along with the placement of replicas.

In the redirect mode, users always access clouds without any replay, so there is no inter-cloud traffic considered by our optimization framework.

\subsubsection{Modeling Reconfiguration Cost}

We define the reconfiguration cost as independent of relay or redirect modes. Different from carbon, operation distance, and inter-cloud traffic, all of which are associated with one data placement, the reconfiguration cost is a measure of the cost incurred by changing one data placement to another. While there may be many ways to define the reconfiguration cost, here we focus on the number of affected users, i.e., those whose masters change locations.

Let $\mathbf{m}_{u}^{\prime}$ denote the location of $u$ 's master in the initial data placement, and $\mathbf{m}_{u}$ denote its location in the optimal one. The reconfiguration cost is

$$
\sum_{u} D_{u}^{r}
$$

where $D_{u}^{r}=\delta\left(\mathbf{m}_{u}^{\prime}, \mathbf{m}_{u}\right)$ simply calculates whether $u$ 's master remains at the cloud where it used to be. We will further extend this definition of the reconfiguration cost later in Section 4.4.3.

\subsubsection{Generalizing Models}

We generalize (4.1) to the intra-cloud cost that is incurred at all clouds. The intra-cloud cost is calculated by replacing $\varepsilon \mathbf{e}_{i}$ in (4.1) with $\alpha_{i}$, where we think of $\alpha_{i}$ as a property (that can be of any dimension) only specific to cloud $i$. The intra-cloud cost can represent the following metrics:

- The carbon footprint as in (4.1), if $\alpha_{i}=\varepsilon \mathrm{e}_{i}$;

- The electricity fees, if $\alpha_{i}=\varepsilon \mathbf{q}_{i}$, where $\mathrm{q}_{i}$ is the per unit electricity price of the region where cloud $i$ is located;

- The fees of using Virtual Machines (VMs) in the clouds, if $\alpha_{i}=\mathrm{Tp}_{i} / \mathrm{M}$, where $\mathrm{T}$ is the time period during which all operations under consideration are executed, $\mathrm{p}_{i}$ is the price per $\mathrm{VM}$ per time unit at cloud $i$, and $\mathrm{M}$ is the number of operations that one VM accommodates during $\mathrm{T}$.

For the relay mode, we generalize $\sum_{u} D_{u}^{d}$ to the user-cloud cost that is incurred between all user-cloud pairs. Replacing $\mathrm{d}_{u, i}$ with $\alpha_{u, i}$, a property only 
specific to user $u$ and cloud $i$, enables the user-cloud cost to represent the following metrics:

- The operation distance between users and clouds, as $\sum_{u} D_{u}^{d}$ in (4.2), if $\alpha_{u, i}=\mathrm{d}_{u, i}$

- The reconfiguration cost as in (4.6), if $\alpha_{u, i}=\delta\left(\mathbf{m}_{u}^{\prime}, i\right) / \mathrm{G}$ where $\mathrm{G}=\mathrm{r}_{u}+$ $\mathrm{w}_{u}+\sum_{v \in \mathbb{N}_{u}}\left(\mathrm{r}_{u v}+\mathrm{w}_{u v}\right)$

- The total network delay or hop counts between users and clouds, if $\alpha_{u, i}=$ $\mathrm{x}_{u, i}$, where $\mathrm{x}_{u, i}$ is the network delay or hop count between $u$ and $i$.

For the redirect mode, by replacing $\mathrm{d}_{u, i}$ with $\alpha_{u, i}$, a property specific to user $u$ and cloud $i$, we generalize $\sum_{u} D_{u}^{d}$ to the user-cloud cost as the cost that is incurred between users and clouds when users access the data of their own and the cost that is incurred by redirection. Similarly, replacing $\mathrm{d}_{u, j}$ with $\alpha_{u, j}$ in $\sum_{u} \sum_{v \in \mathbb{N}_{u}} V_{u \rightarrow v}^{d}{ }^{\prime}$ generalizes it to the user-cloud cost that is incurred between users and clouds when users access the data of others.

- $\sum_{u} D_{u}^{d}$ can represent $\sum_{u} D_{u}^{d^{\prime}}$ as in (4.4), if $\alpha_{u, i}=\mathrm{d}_{u, i}\left(\left(\mathrm{r}_{u}+\mathrm{w}_{u}+1\right) / \mathrm{G}\right)$ where $\mathrm{G}$ is explained above.

We finally generalize $\sum_{u} \sum_{v \in \mathbb{N}_{u}} V_{u \rightarrow v}^{d}$ to the inter-cloud cost that is incurred between all pairs of clouds. It is calculated by using $\alpha_{i, j}$ that is specific to a pair of clouds $i, j$ to replace $\mathrm{d}_{i, j}$ in $\sum_{u} \sum_{v \in \mathbb{N}_{u}} V_{u \rightarrow v}^{d}$. The inter-cloud cost can represent the following metrics:

- The operation distance between clouds, as $\sum_{u} \sum_{v \in \mathbb{N}_{u}} V_{u \rightarrow v}^{d}$ in (4.2), if $\alpha_{i, j}=\mathrm{d}_{i, j}$

- The inter-cloud traffic as in (4.5), if $\alpha_{i, j}=\mathrm{t}$;

- The total inter-cloud network delay or hop counts, similarly.

Our generalized models potentially cover a wide range of metrics that can be used as system objectives of the multi-cloud socially aware service, going beyond the concerns of cloud customers, i.e., socially aware service providers in our case. The electricity fee is, for instance, not of the interest of cloud customers as they do not directly pay for it, but rather a concern of cloud or data center operators who can also leverage our models to seek optimization. One can explore our models to express even more metrics in the multi-cloud environment. 


\subsection{Problem}

Based on (4.1), (4.2), (4.3), (4.4), (4.5), (4.6), and putting all objectives together, we minimize the following cost function:

$$
\begin{array}{r}
\sum_{u} D_{u}\left(\mathbf{m}_{u}, \mathbf{s}_{u, 1}, \ldots, \mathbf{s}_{u, \mathrm{k}}\right)+ \\
\sum_{u} \sum_{v \in \mathbb{N}_{u}} V_{u \rightarrow v}\left(\mathbf{m}_{u}, \mathbf{m}_{v}, \mathbf{s}_{v, 1}, \ldots, \mathbf{s}_{v, \mathrm{k}}\right),
\end{array}
$$

where

$$
D_{u}=D_{u}^{c}+D_{u}^{d}+D_{u}^{r}, V_{u \rightarrow v}=V_{u \rightarrow v}^{c}+V_{u \rightarrow v}^{d}+V_{u \rightarrow v}^{t}
$$

in the relay mode, and

$$
D_{u}=D_{u}^{c}+D_{u}^{d^{*}}+D_{u}^{r}, V_{u \rightarrow v}=V_{u \rightarrow v}^{c}+V_{u \rightarrow v}^{d}{ }^{\prime}
$$

in the redirect mode. Recall that in Section 4.2.3, we consider two cases of the redirect mode: $D_{u}^{d^{*}}=D_{u}^{d}$ if every read/write request needs a redirection in prior, and $D_{u}^{d^{*}}=D_{u}^{d^{\prime}}$ if a user only needs to contact her master cloud once for all later $\mathrm{read} /$ write requests.

Our formulation features the optimization problem in the following structure: $D_{u}$ only depends on the locations of a single user's data (her master and slaves), and $V_{u \rightarrow v}$ depends on the locations of a pair of neighboring users' data (the master of user $u$ who conducts operations and the master and slaves of user $v$ who receives operations). We refer to the former as the unary cost, as it contains the decision variables of only one user, and the latter as the pairwise cost, as it contains the decision variables of a pair of users.

Note that (4.8) is equivalent to the sum of the three types of costs defined in Section 4.2.6, with $\alpha_{i}=\varepsilon \mathrm{e}_{i}, \alpha_{u, i}=\mathrm{d}_{u, i}+\delta\left(\mathbf{m}_{u}^{\prime}, i\right) / \mathrm{G}$, and $\alpha_{i, j}=\mathrm{d}_{i, j}+\mathrm{t}$; (4.9) is also equivalent to the sum of the three types of costs with $\alpha_{i}=\varepsilon \mathrm{e}_{i}$, $\alpha_{u, i}=\mathrm{d}_{u, i}+\delta\left(\mathbf{m}_{u}^{\prime}, i\right) / \mathrm{G}$ and $\alpha_{u, j}=\mathrm{d}_{u, j}$ for the first case of redirect mode, with $\alpha_{i}=\varepsilon \mathrm{e}_{i}, \alpha_{u, i}=\left(\mathrm{d}_{u, i}\left(\mathrm{r}_{u}+\mathrm{w}_{u}+1\right)+\delta\left(\mathbf{m}_{u}^{\prime}, i\right)\right) / \mathrm{G}$ and $\alpha_{u, j}=\mathrm{d}_{u, j}$ for the second case of redirect mode. Due to the generality of the three types of costs, solving our optimization problem with (4.8) and (4.9) implies solving a class of problems with a variety of system objectives in the relay and the redirect modes.

One can associate a weight with each objective, or with each of the three types of costs. For the ease of presentation, we do not write such weights in all our formulas. One can tune these weights by standard approaches in order to seek trade-offs among objectives based on one's own requirements.

As a multi-objective optimization problem, our problem is mainly subject to the constraint of no co-location of a user's replicas at a common cloud, i.e., $\mathbf{m}_{u} \neq \mathbf{s}_{u, l}, \mathbf{s}_{u, l} \neq \mathbf{s}_{u, l^{\prime}}$, where $l, l^{\prime}=1, \ldots, \mathrm{k}, l \neq l^{\prime}, \forall u$. Note that we do not regard cloud capacity as a constraint for the following reasons: (1) From a cloud customer's perspective, a cloud can provide "infinite" resources on demand; (2) 
By assuming an unlimited capacity of each cloud, we aim at a lower bound of the total cost possible with our framework.

\subsection{Algorithm}

This section describes how we solve the data placement problem defined in (4.7). This problem is intractable due to its NP-hardness; it is difficult to solve due to its discrete, combinatorial nature. For $N$ clouds and $M$ users where every user has $\mathrm{k}$ slaves, the size of the solution space is $\left(\frac{N !}{\mathrm{k} !(N-\mathrm{k}-1) !}\right)^{M}$, which is huge for real world services with a very large user base. Our general idea is developing practical and efficient heuristics to seek good approximate solutions.

\subsubsection{Decoupling Masters from Slaves}

Based on our observation of the problem's structure as stated in Section 4.3, we come up with two insights as follows.

Insight 1: The difficulty in optimizing (4.7) roots partially in that the decisions of placing master replicas and slave replicas affect each other. If we can "split" the master placement and the slave placement, it may be easier to solve each of them.

Insight 2: Our problem may be potentially connected to the minimal $s$ $t$ cut problem [39]. Intuitively and naively, consider two clouds and a social network, and let's assign users to clouds. We can add edges to the social graph by connecting every user with every cloud, regarding one cloud as the $s$ terminal and the other as the $t$ terminal. The weight of the edge between any two users is the pairwise cost of assigning them to different clouds, and the weight of the edge between a user and a cloud is the unary cost of assigning this user to this cloud. Consequently, by finding the minimal $s$ - $t$ cut of this graph, we find the set of edges with the minimum total cost, where each edge between a user and a cloud represents the optimal assignment of the user to the corresponding cloud.

Motivated by these two insights, we decompose our data placement problem into two subproblems.

Master replicas placement: The first subproblem is placing users' master replicas given the placement of users' slave replicas, which is formulated as minimizing

$$
\sum_{u} D_{u}\left(\mathbf{m}_{u}\right)+\sum_{u} \sum_{v \in \mathbb{N}_{u}} V_{u \rightarrow v}\left(\mathbf{m}_{u}, \mathbf{m}_{v}\right)
$$

Leveraging a more sophisticated version of iterative minimal $s$ - $t$ cuts $[25,26,56]$ as will be described in Section 4.4.2, we can identify the optimal assignments of users' masters to clouds.

Slave replicas placement: The second subproblem is placing users' slave replicas given the placement of users' master replicas, which is formulated as 
minimizing

$$
\sum_{u} D_{u}\left(\mathbf{s}_{u, 1}, \ldots, \mathbf{s}_{u, \mathrm{k}}\right)+\sum_{u} \sum_{v \in \mathbb{N}_{u}} V_{u \rightarrow v}\left(\mathbf{s}_{v, 1}, \ldots, \mathbf{s}_{v, \mathrm{k}}\right) .
$$

As will be shown in Section 4.4.3, minimizing the total cost incurred by all users' slaves can be achieved by independently minimizing the cost incurred by each user's slaves.

Overall, our approach consists of solving the two subproblems of minimizing (4.10) and (4.11) alternately via fixed-point iterations, as in Fig. 4.3. Starting with an initial placement of all masters and slaves, we solve the two subproblems iteratively to reduce the total cost and to improve the solution of each subproblem, until no further cost reduction is possible or until an expected number of iterations are executed.

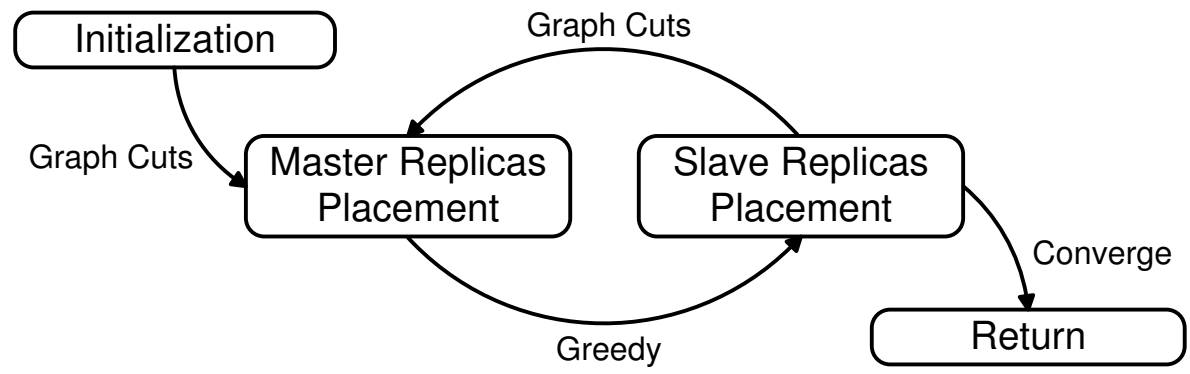

Figure 4.3: Our fixed-point iteration approach

\subsubsection{Solving Master Placement by Graph Cuts}

The idea is iterating cloud pairs and finding the minimal $s$ - $t$ cut for each pair [26]. Every minimal $s$ - $t$ cut represents the optimal assignments of involved masters to a pair of clouds. The algorithm keeps iterating cloud pairs to update the assignments until the total cost of the assignments of all masters cannot be reduced any more.

Fig. 4.4 visualizes how this works. Users, i.e., their master replicas, are in the middle while at the top and the bottom are the clouds. An edge between two users means the two users have interactions. An edge between a user and a cloud indicates that the user's master is placed at that cloud. Initially, every user is connected to a cloud arbitrarily. Selecting a cloud pair, e.g., the blue ones in this figure, it constructs a graph by connecting both clouds to every user who is connected to one of the two clouds, as in the left part of Fig. 4.4, and assigns an appropriate weight to each edge in this constructed graph. The weight of the edge between a user $u$ and a cloud $i$ is computed based on $D_{u}(i)$, reflecting how much $u$ wants her master to be placed at $i$, while the weight of the edge between two users $u$ and $v$ is computed based on $V_{u \rightarrow v}(i, j)+V_{v \rightarrow u}(j, i)$, reflecting how 
much $u$ and $v$ want their masters to be placed at $i$ and $j$, respectively. We can always find the minimal $s$ - $t$ cut of the constructed graph by the max-flow algorithm [39]. The right part of Fig. 4.4 marks the cut edges by double dashes, where other edges of the constructed graph that are not in the cut set are not shown. The algorithm continues by selecting another cloud pair, e.g., a blue one and the red one, constructs a graph by adding edges, and calculates the minimal $s-t$ cut for the optimal assignments of involved users to these two clouds, etc.

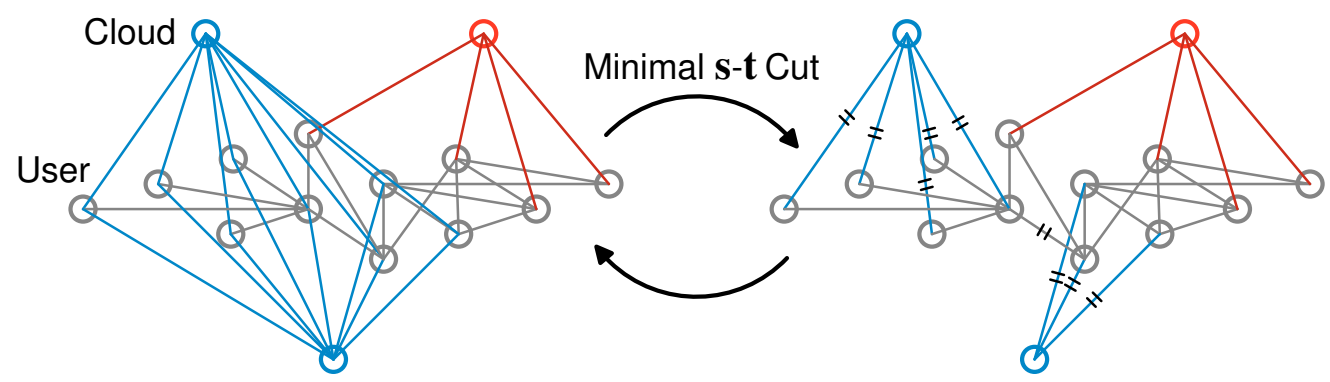

Figure 4.4: Using graph cuts to place master replicas

\subsubsection{Solving Slave Placement by a Greedy Approach}

Note that we can use $\sum_{u} \sum_{v \in \mathbb{N}_{u}} V_{u \rightarrow v}\left(\mathbf{s}_{v, 1}, \ldots, \mathbf{s}_{v, \mathrm{k}}\right)=\sum_{u} \sum_{v \in \mathbb{N}_{u}} V_{v \rightarrow u}\left(\mathbf{s}_{u, 1}, \ldots, \mathbf{s}_{u, \mathrm{k}}\right)$ to transform (4.11) into

$$
\sum_{u}\left(D_{u}\left(\mathbf{s}_{u, 1}, \ldots, \mathbf{s}_{u, \mathrm{k}}\right)+\sum_{v \in \mathbb{N}_{u}} V_{v \rightarrow u}\left(\mathbf{s}_{u, 1}, \ldots, \mathbf{s}_{u, \mathrm{k}}\right)\right),
$$

implying that slave data placements for different users are independent, given the locations of all users' masters. Therefore, we can solve this subproblem by finding the optimal placement of the slave replicas of each user separately.

A greedy method to place a user's slave replicas finds the current best cloud for a slave replica, places it there, and repeats doing this until all the $k$ slaves of this user are placed. The cost incurred by placing place $u$ 's $l$ th $(2 \leq l \leq \mathrm{k})$ slave at cloud $i\left(i \neq \mathbf{m}_{u}, i \neq \mathbf{s}_{u, l^{\prime}}, l^{\prime}=1, \ldots, l-1\right)$ is $\gamma_{u, i}=\alpha_{i}\left(\mathrm{w}_{u}+\sum_{v \in \mathbb{N}_{u}} \mathbf{w}_{v u}\right)+$ $\left.\beta_{u, i}\right|_{z_{v, u}^{\prime \prime \prime}}-\left.\beta_{u, i}\right|_{z_{v, u}^{\prime \prime}}$, where $\beta_{u, i}=\sum_{v \in \mathbb{N}_{u}}\left(\left(\alpha_{z_{v, u}}+\alpha_{\mathbf{m}_{v}, z_{v, u}} \delta\left(\mathbf{m}_{v}, z_{v, u}\right)\right) \mathbf{r}_{v u}\right)$ in the relay mode and $\beta_{u, i}=\sum_{v \in \mathbb{N}_{u}}\left(\left(\alpha_{z_{v, u}}+\alpha_{v, z_{v, u}}\right) \mathbf{r}_{v u}\right)$ in the redirect mode, $\alpha$. and $\alpha$., are as explained in Section 4.2.6. $\left.\beta_{u, i}\right|_{z_{v, u}^{\prime \prime \prime}}$ means calculating $\beta_{u, i}$ by replacing $z_{v, u}$ with $z_{v, u}^{\prime \prime \prime}=z_{v, u}\left(\mathbf{s}_{u, 1}, \ldots, \mathbf{s}_{u, l-1}, i\right)$, and $\left.\beta_{u, i}\right|_{z_{v, u}^{\prime \prime}}$ means calculating $\beta_{u, i}$ by replacing $z_{v, u}$ with $z_{v, u}^{\prime \prime}=z_{v, u}\left(\mathbf{s}_{u, 1}, \ldots, \mathbf{s}_{u, l-1}\right) . z_{v, u}^{\prime \prime \prime}$ returns the selected cloud if $u$ 's $l$ th slave is placed at cloud $i$, and $z_{v, u}^{\prime \prime}$ returns the selected cloud when this slave does not exist in the system. Therefore, the best cloud for $u$ 's $l$ th slave is $i=\arg \min _{i} \gamma_{u, i}$. When $l=1$, we naturally do not have $\left.\beta_{u, i}\right|_{z_{v, u}^{\prime \prime}}$ and only use $\left.\beta_{u, i}\right|_{z_{v, u}^{\prime \prime \prime}=i}$ in $\gamma_{u, i}$.

An exhaustive method is also possible. Except a user's master cloud, one can select $k$ clouds out of all other clouds to place this user's k slaves, and 
after checking all the possibilities, place slaves on those where the total incurred cost is minimal. This exhaustive approach always finds the theoretical/global minimum. However, compared with the aforementioned greedy method with a time complexity of $O(\mathrm{k} N)$, the exhaustive approach runs at $O\left(N^{\mathrm{k}} /(\mathrm{k}-1) !\right)$, where $N$ is the total number of clouds.

With all the knowledge so far about slave replicas, we can extend $\gamma_{u, i}$ to include $\delta_{u}^{\prime}(i)$ as part of it, where $\delta_{u}^{\prime}(i)=0$ if $i \in\left\{\mathbf{s}_{u, 1}^{\prime}, \ldots, \mathbf{s}_{u, \mathrm{k}}^{\prime}\right\}$ and $\delta_{u}^{\prime}(i)=1$ otherwise, and $\mathbf{s}_{u, l}^{\prime}, l=1, \ldots, \mathrm{k}$, are $u$ 's slave locations in the initial placement. $\delta_{u}^{\prime}(i)$, jointly with (4.6), enables us to calculate the total number of moved masters and slaves as the reconfiguration cost.

\subsection{Evaluations}

With real-world traces, we do extensive evaluations in a variety of realistic settings. We demonstrate both the optimization results and the algorithm performance. In the former, we find that our approach achieves significantly better results than existing approaches, and can explore trade-offs among objectives; in the latter, we see that our approach converges fast, scales to a huge user base, and the choices of initial placements and slave placements can influence the optimization results to different extents.

\subsubsection{Data Preparation}

Users: We obtained 107,734 users all across the US with 2,744,006 social relations among them by crawling Twitter in a breadth-first manner in 2010. We translate each user's profile location into geographic coordinates (i.e., [latitude, longitude]), enabling us to calculate the geographic distance. Our Twitter graph is a single connected component, and is used as an undirected social graph throughout our evaluations, as in [72]. Fig. 4.5 is a CDF, showing that about $30 \%$ of social relations in our dataset stretch within $500 \mathrm{~km}$ and all the rest spread almost uniformly over geographic distance.

Clouds: According to the geographic distribution of users, we select 10 regions across the US and select a city out of each region as the location of a cloud. Fig. 4.5 confirms that our clouds are at or near locations with dense user populations, as about $80 \%$ of users can find a cloud closest to them with no longer than $500 \mathrm{~km}$. Fig. 4.6 indicates that $30 \%$ of all friends of a user have the same closest cloud as the user. Considering more clouds, we see that, e.g., the 5 closest clouds to a user can include the closest cloud to about $60 \%$ of all the user's friends. The default factors for estimating carbon emissions are also reported on a per region basis[9]. Table 4.1 lists the cities we select in a carbon ascending order. 


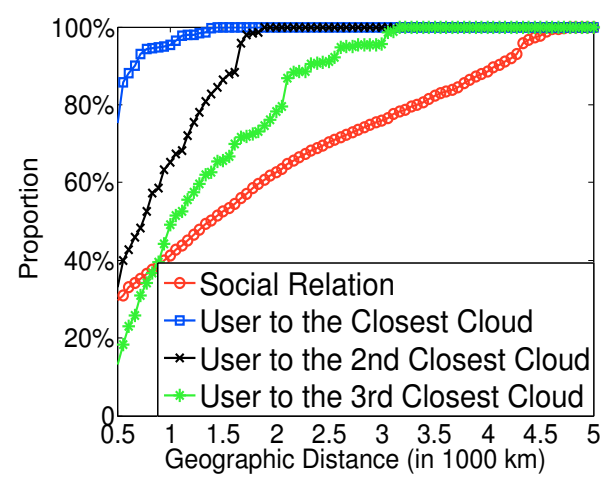

Figure 4.5: Geo-distribution of users

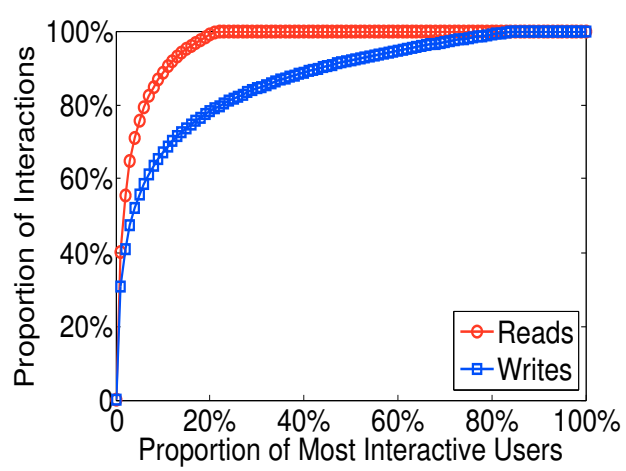

Figure 4.7: Interaction distribution over users

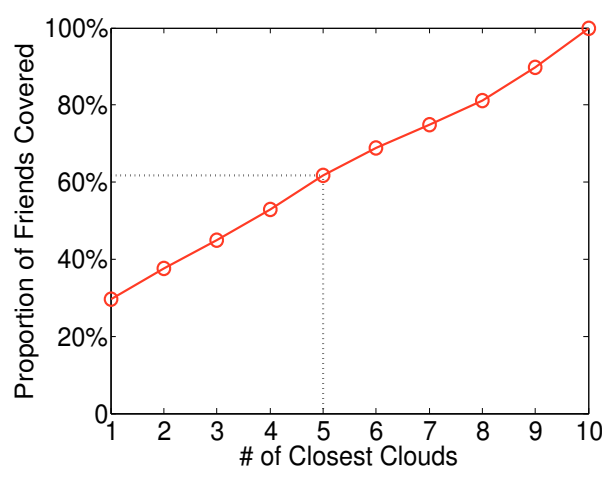

Figure 4.6: Geo-distribution of friends

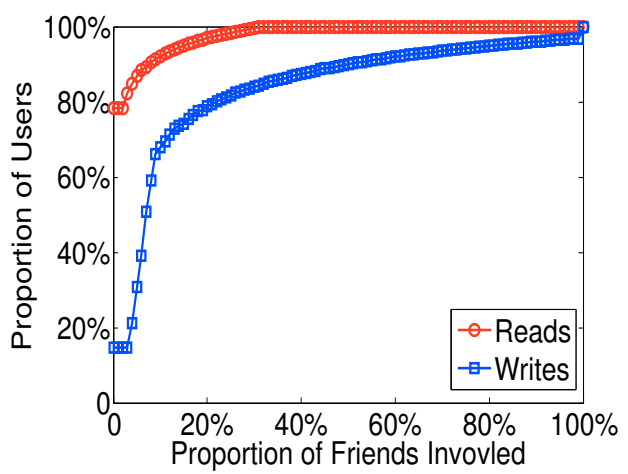

Figure 4.8: Interaction distribution over friends

Interactions: It is extremely hard to obtain interaction traces, especially for read operations such as one user browsing another user's profile. Service providers are often reluctant to share such data due to competition and privacy concerns [65]. User interactions differ from other types of cloud workloads in how read and write operations are distributed among users and among users' friends. Recent literature, fortunately, disclosed such features for a small-scale and local OSN [48, 90], making it possible to synthesize realistic user interactions. Fig. 4.7 and 4.8 provide CDF distributions of the operations synthesized among our realworld Twitter users. Without loss of generality, we have precisely scaled the OSN interactions of the smaller, regional OSN to our large-scale, geo-distributed OSN.

We highlight how we capture the distributions of real-world user interactions and achieve the aforementioned realistic synthesization with our social graph. We first do curve fitting for the distributions of reads and writes among top interactive users, and the distributions of reads and writes among each user's friends reported in [48], and use these fitted curves as inputs. With a given total number of reads and that of writes conducted by all users, we then perform the following steps: (1) Sort all users in the descending order of social degree. With the reads and writes distributions among top interactive users, calculate for each 
Table 4.1: Carbon intensities of cloud locations

\begin{tabular}{|c|c|c|c|}
\hline City & State & eGRID Region [9] & $\begin{array}{c}\mathrm{CO}_{2} \\
(\mathrm{lb} / \mathrm{MWh})\end{array}$ \\
\hline Palo Alto & CA & WECC California & 658.68 \\
\hline Fall River & MA & NPCC New England & 728.41 \\
\hline Seattle & WA & WECC Northwest & 819.21 \\
\hline Secaucus & NJ & RFC East & 947.42 \\
\hline Ashburn & VA & SERC Virginia/Carolina & 1035.87 \\
\hline Miami & FL & FRCC All & 1176.61 \\
\hline San Antonio & TX & ERCOT All & 1181.73 \\
\hline Atlanta & GA & SERC South & 1325.68 \\
\hline Lansing & MI & RFC Michigan & 1659.46 \\
\hline St. Louis & MO & SERC Midwest & 1749.75 \\
\hline
\end{tabular}

user the number of reads and that of writes that this user conducts to her neighbors. (2) With the sorted users and the distributions of reads and writes among each user's friends, calculate for each user the number of neighbors that this user reads and writes. (3) For each user, select the specified number (as calculated in the previous step) of neighbors, and for a user's each selected neighbor, assign the number of reads and writes conducted by this user to this neighbor as being proportional to this neighbor's social degree (as in the preferential model [45]). Note that we do not consider users' geographical locations during this procedure, as location does not obviously influence interactions [51].

\subsubsection{Experimental Settings}

Interaction workload: As stated in the previous section, we can control the total number of reads and that of writes to produce different workloads while always maintaining the featured distributions of interactions. We use the ratio of the total number of reads over that of writes " $\mathrm{R} / \mathrm{W}$ " to denote workloads. We evaluate the case of " $\mathrm{R} / \mathrm{W}=10$ ", to reflect the fact that OSN services have many more reads than writes $[48,60]$, and the case of " $\mathrm{R} / \mathrm{W}=1$ ", to investigate how "R/W" affect the benefits of our approach.

Number of slaves: We have 10 clouds, and we evaluate 8 cases by iterating the number of slave replicas per user from 1 to 8 . The number of slaves per user depends on a lot of factors, e.g., data availability requirements, the monetary budget of the service provider, etc. We do not intend to decide what number is the best for a provider. Here what we want to check is whether our solution is better in all objective dimensions than existing approaches for any given number of slaves per user.

Multi-cloud access policies: We evaluate some policies that we consider would be the most practical ones in reality. For the relay mode, based on (4.12) 
in Section 4.6.1, we evaluate the "Master" policy where $z_{u, v}^{\prime}=\mathbf{m}_{v}$, and the "Closest" policy where $z_{u, v}^{\prime}=\arg \min _{i}\left(\mathrm{~d}_{\mathbf{m}_{u}, i}\right), \forall i \in\left\{\mathbf{m}_{v}, \mathbf{s}_{v, 1}, \ldots, \mathbf{s}_{v, \mathrm{k}}\right\}$. For the redirect mode, we evaluate the "Closest" policy where $z_{u, v}=\arg \min _{i}\left(\mathrm{~d}_{u, i}\right)$, $\forall i \in\left\{\mathbf{m}_{v}, \mathbf{s}_{v, 1}, \ldots, \mathbf{s}_{v, \mathrm{k}}\right\}$ for both cases of "redirect only once" and "redirect every time". We do not want to decide which the best access policy can be; instead, we want to check for every given policy whether our approach is consistent in optimizing all objectives.

Weights of multi-objectives: We have four objectives to optimize and thus four weights. We vary the weights to seek trade-offs among objectives, and use the ratio of weights to denote our variations. Note that we have normalized all dimensions of inputs to the same order of magnitude in value, and the ratios reported throughout the evaluations are the ones of weights associated with the normalized inputs.

Figure settings: Table 4.2 summarizes the specific settings corresponding to each figure. "A/F" means that a figure is varying this setting for comparison, and relevant information is available in the figure itself. Note that we do not consider the reconfiguration cost except in Fig. 4.18, thus the ratios in this table are between three weights rather than four; other information about settings that is not summarized in this table is mentioned along with the interpretation of each figure.

Table 4.2: Evaluation settings for figures

\begin{tabular}{|c|c|c|c|c|c|}
\hline Fig. & R/W & Slave \# & Policy & Weights & Mode \\
\hline $\begin{array}{c}4.11,4.12,4.19 \\
4.22,4.24\end{array}$ & 10 & $\mathrm{~A} / \mathrm{F}$ & Closest & $1: 1: 1$ & Relay \\
\hline $4.9,4.10,4.13$ & 10 & $\mathrm{~A} / \mathrm{F}$ & $\mathrm{A} / \mathrm{F}$ & $1: 1: 1$ & Relay \\
\hline $4.14,4.15$ & 10 & $\mathrm{~A} / \mathrm{F}$ & $\mathrm{A} / \mathrm{F}$ & $1: 1: 1$ & Redirect \\
\hline 4.16 & $\mathrm{~A} / \mathrm{F}$ & 4 & $\mathrm{~A} / \mathrm{F}$ & $1: 1: 1$ & Relay \\
\hline 4.17 & 10 & 4 & Closest & $\mathrm{A} / \mathrm{F}$ & Relay \\
\hline $\begin{array}{c}4.18,4.20 \\
4.21,4.23\end{array}$ & 10 & 4 & Closest & $1: 1: 1$ & Relay \\
\hline
\end{tabular}

Algorithmic settings: We implement different placement methods by $\mathrm{C}++$. In particular, as for our proposed approach to solve master data placement, we calculate various costs according to our models and feed them to the gco-v3.0 library $[25,26,56]$, an open source implementation of graph cuts. We invoke this library with the option of $\alpha$ - $\beta$-swap [26].

\subsubsection{Optimization Results}

How much benefit can we gain? We compare the data placements produced by our approach with those produced by random placement, the standard 


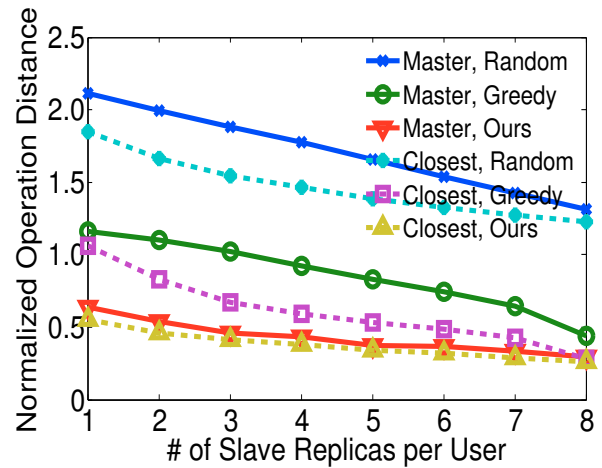

Figure 4.9: Operation distance

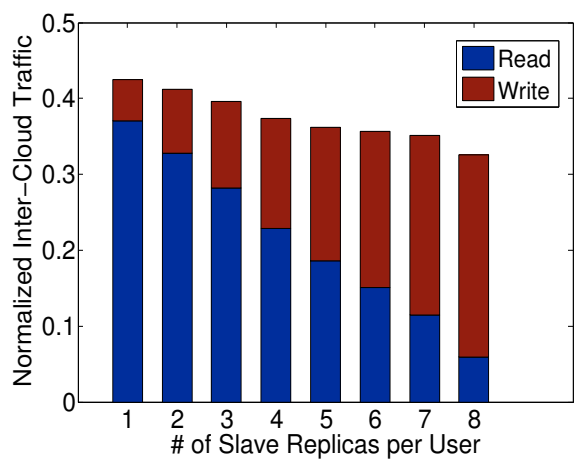

Figure 4.11: Greedy traffic

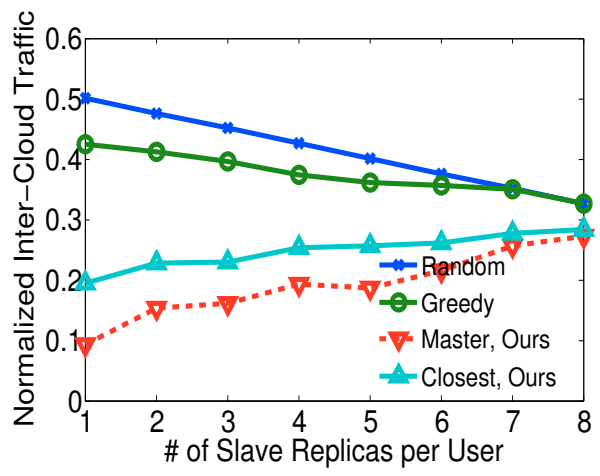

Figure 4.10: Inter-cloud traffic

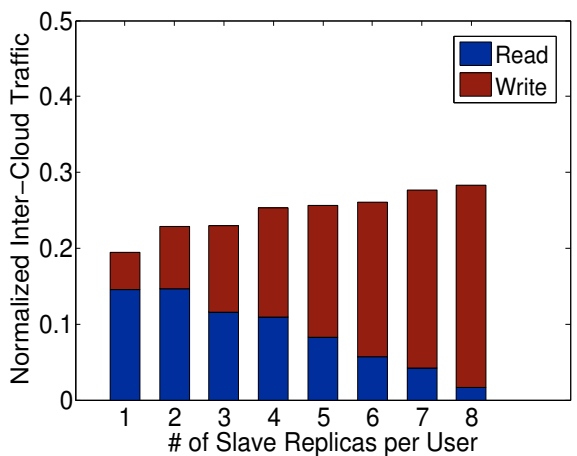

Figure 4.12: Our traffic

practice of distributed databases (e.g., MySQL) and key-value stores (e.g., Cassandra), and by greedy placement, the de facto practice of many real-world services $[80,82]$. The random approach places each replica of a user randomly at one of the clouds. The greedy approach places a user's master at the closest cloud to that user, and places her $\mathrm{k}$ slaves at the other closest $\mathrm{k}$ clouds to that user. As in Fig. 4.3, our approach uses greedy placement at initialization.

Fig. 4.9 shows the operation distance of different data placements. The distance always drops as a user has more slaves, since data become available at more clouds and more operations can be completed locally or nearby. Greedy beats random because slaves randomly placed at clouds are less likely to benefit friends, due to the locality shown in Fig. 4.6. Our approach beats both random and greedy. Across all cases, we save 33\%-54\% distance when the master policy is applied, and 7\%-48\% when the closest policy is applied, compared with greedy. We save even more compared with random. The benefit of our approach over others roughly decreases as the slave number increases, because the number of clouds that do not have a user's replica becomes smaller and less room is left for optimization by rearranging replica locations.

Fig. 4.10 depicts the inter-cloud traffic (including those incurred by the propagated writes) of different data placements. In the random and greedy placements, 


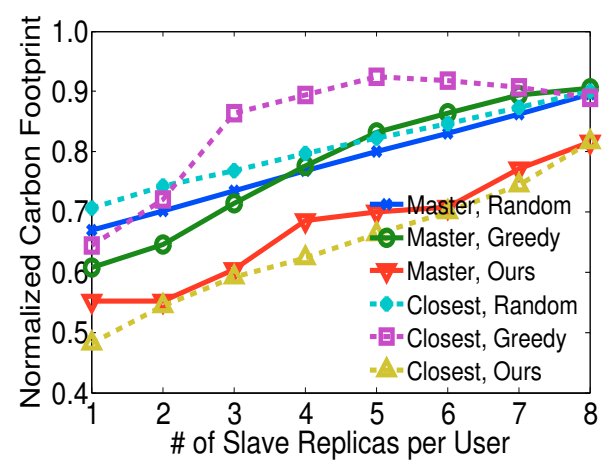

Figure 4.13: Carbon footprint

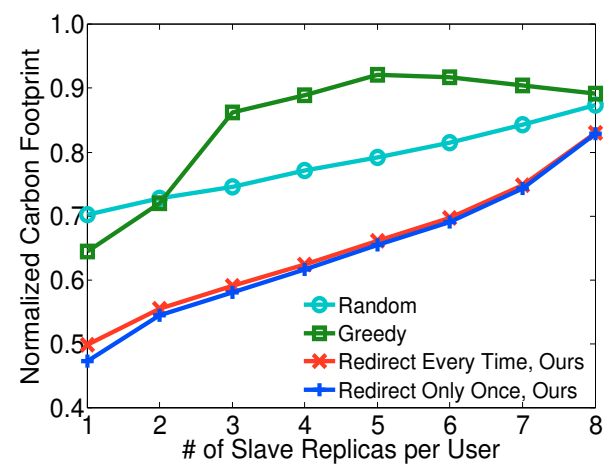

Figure 4.15: Carbon footprint

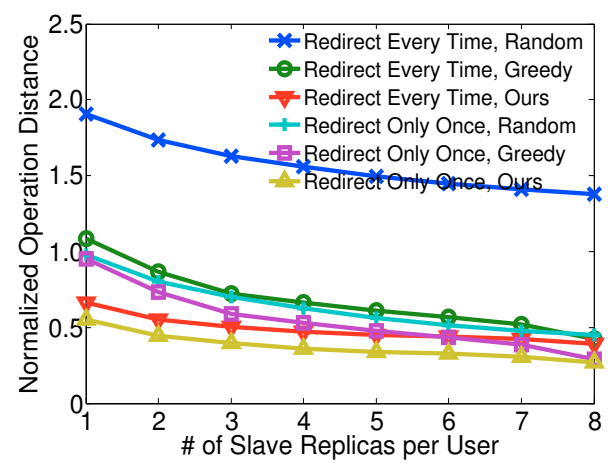

Figure 4.14: Operation distance

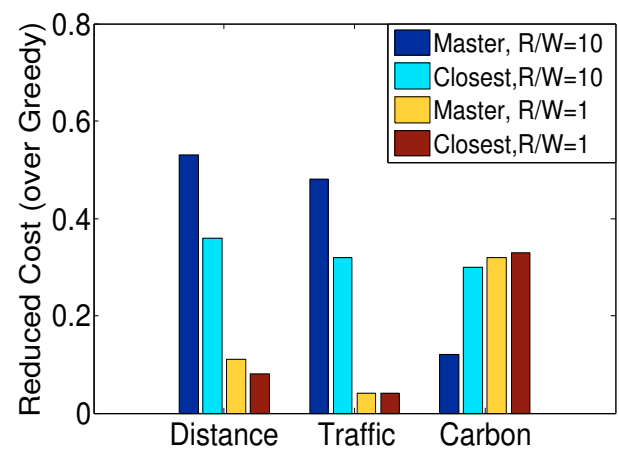

Figure 4.16: Influence of workload

the amount of traffic does not depend on access policies. With our approach, using different policies as inputs leads to different placements and thus different amounts of traffic. Our data placements have 13\%-78\% less traffic than others. We dissect the traffic details in Fig. 4.11 and 4.12, where we show greedy and our approach with the closest policy as examples. The growth of the number of slaves per user incurs more write traffic to maintain consistency, while the amount of read traffic becomes less due to the increased data availability at more clouds. Overall, random and greedy have the total traffic descend; the traffic of our solutions keeps increasing, as we reduce the read traffic by a large fraction and the write traffic becomes a dominance.

Fig. 4.13 focuses on the carbon footprint. Our approach saves 10\%-30\% carbon compared to random and greedy. The essential feature that distinguishes carbon from distance and traffic is that both the latter encourage data to be placed closely, as stated in Section 4.6.2; carbon does not necessarily favor this, but rather depends on at which clouds the operations are executed. Random has a steadily growing carbon as the slave number increases, since it tends to span each user's replicas all across the clouds with carbon intensity also spanning a certain range, as in Table 4.1. Greedy's carbon changes up and down since it places data collectively and tends to always use a set of nearby clouds. 
Fig. 4.14 and 4.15 visualize the operation distance and the carbon footprint for the redirect mode, respectively. Overall, we observe similar trends compared with the relay mode. Our approach always has smaller distance and lower carbon footprint than random and greedy. It is natural than redirect every time has larger distance than redirect only once. Random placement with redirect every time has much larger distance than other cases; in the greedy case, as every user's master cloud is the closest to a user, adding an access to one's master cloud before one's every access to friends' data does not lead to too much additional operation distance on the whole. This is also the reason why our approach cannot optimize that much as for the relay mode, as there is not so much space for optimization.

How does the workload influence the benefit? Fig. 4.16 describes how the total number of reads and writes among users may influence the advantages of our approach. For both policies, our approach optimizes distance and traffic more than carbon, when there are more reads than writes, and vice versa. This is normal, because the advantage of our approach lies in optimizing reads, and writes excluding the propagated ones. More writes imply more propagations, leaving the system with less room for optimization, which, in turn, indicates that our approach is more suitable and capable for read-intensive services like socially aware ones and many others.

What are the trade-offs among objectives? Fig. 4.17 indicates that, by tuning the weight of each objective, one can seek a range of trade-offs without changing any other part of our framework. Here we choose to tune distance and traffic as an example while fixing the weight of carbon. We set the ratio of the distance weight over the traffic weight to be (1) 1:1, (2) 10:1, (3) 1:10, and (4) 10:10. We make the following observations: (2) has a larger distance weight than (1), and thus (2) is smaller in distance, and is in turn larger in traffic and carbon; (3) has a larger traffic weight than (1), and thus (3) is smaller in traffic and larger in distance and carbon; (4) has larger distance and traffic weights than (1), and is thus smaller in these two dimensions and larger in carbon.

Fig. 4.18 additionally considers the reconfiguration cost, i.e., the total number of moved masters and slaves. By controlling the weight of the reconfiguration cost, one can set it as cheap or expensive to move replicas across clouds. In this figure, we set this weight to be 1 and 10, respectively. An interesting observation is setting it to be 10 times larger can efficiently prohibit replica movements across clouds. However, this does not prevent optimization, as the 1 case moves a huge many more replicas and only optimizes about $30 \%$ more than the 10 case.

\subsubsection{Algorithm Performance}

How fast does our approach converge? Fig. 4.19 illustrates the total costs of the data placement after each iteration of our approach, varying the number 


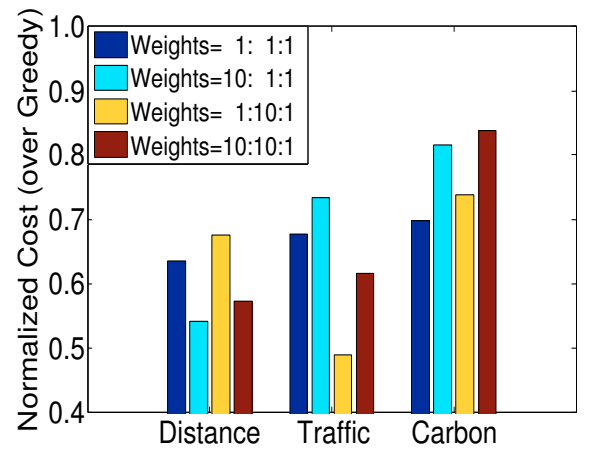

Figure 4.17: Tuning weights

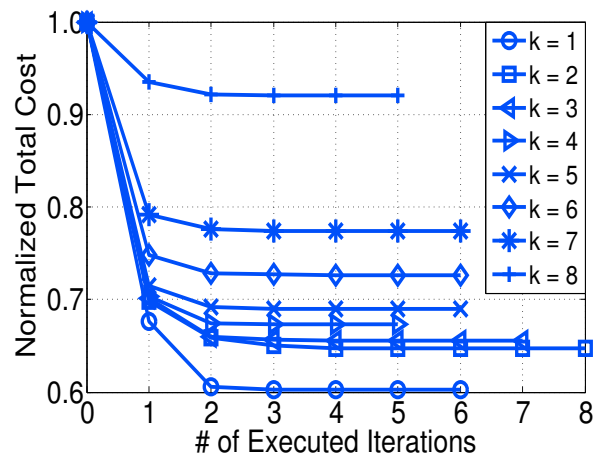

Figure 4.19: Convergence

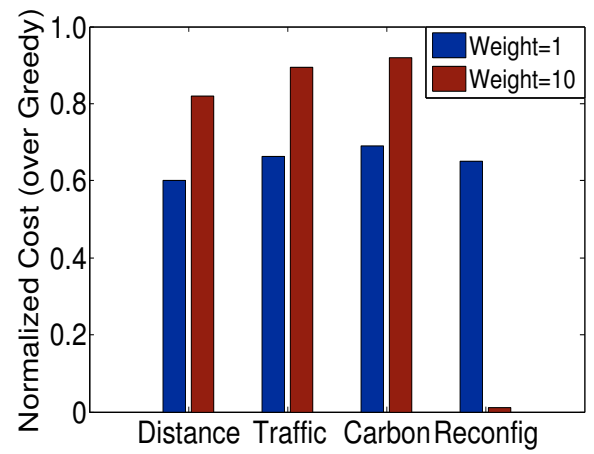

Figure 4.18: Reconfiguration

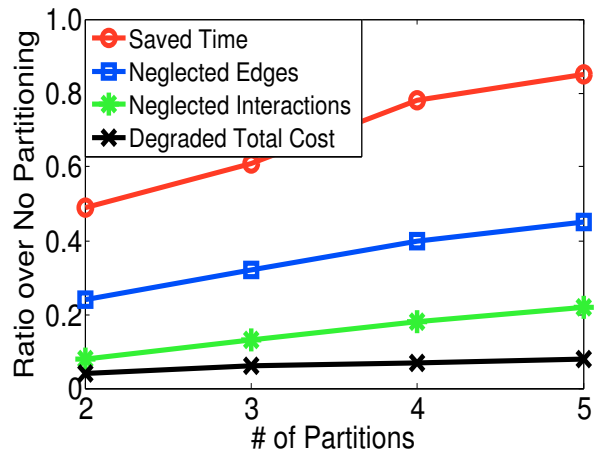

Figure 4.20: Scalability

of slaves per user. One iteration includes an execution of graph cuts to solve the master replicas placement and an execution of our greedy method to solve the slave replicas placement. This figure indicates that the most cost reduction is achieved in the first iteration. For all cases, the largest number of required iterations is 8 , after which no cost can be reduced any more. Our approach is highly efficient and converges fast. In practice, one can even adopt an early stop strategy, i.e., running 2 or 3 iterations and terminating the algorithm is sometimes already sufficient to achieve a large part of optimization.

How scalable is our approach? Fig. 4.20 demonstrates the scalability of our approach. We use METIS [53] to partition our original dataset into several partitions, and then apply our approach to each partition independently while neglecting the inter-partition interactions. Doing so saves up to $85 \%$ (in the 5 -partition case) of the total execution time, and only degrades the total cost of the optimal data placement by less than $8 \%$, compared with running our approach directly on the original dataset. This success roots in the community structure of OSN social relations and interactions; thus even neglecting $45 \%$ social relations and the associated $22 \%$ interactions of the original dataset only has a slight influence on the optimization. For real-world data with a stronger community structure, we expect even less cost degradation. 


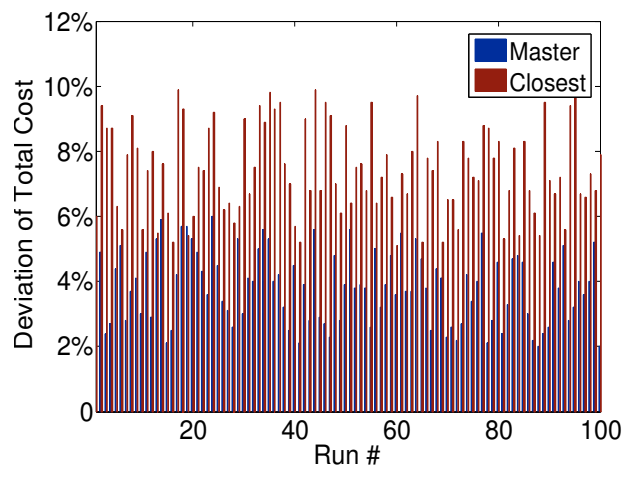

Figure 4.21: Influence of initial placements on cost

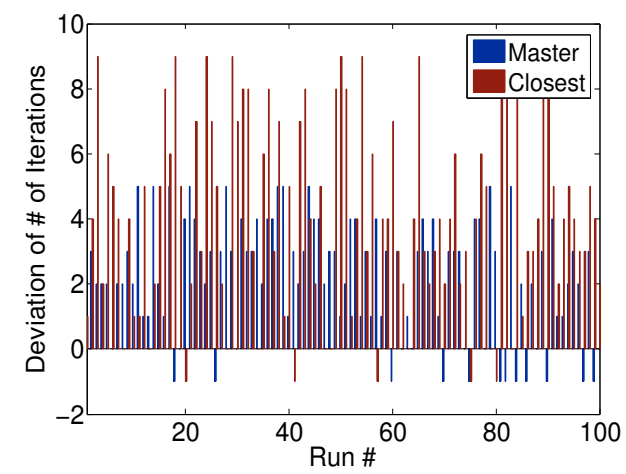

Figure 4.23: Influence of initial placements on iteration \#

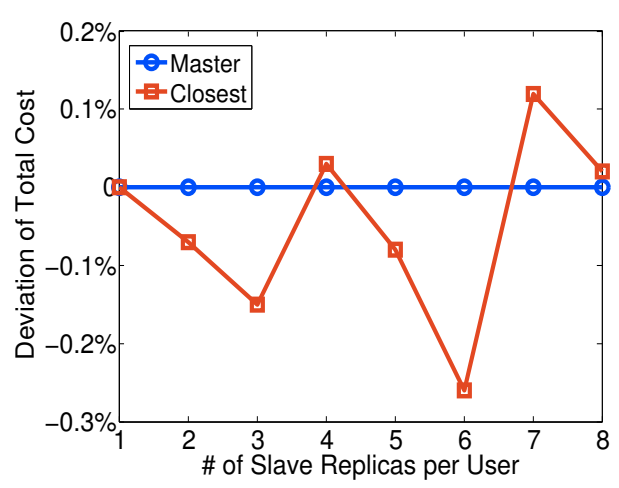

Figure 4.22: Influence of slave placements on cost

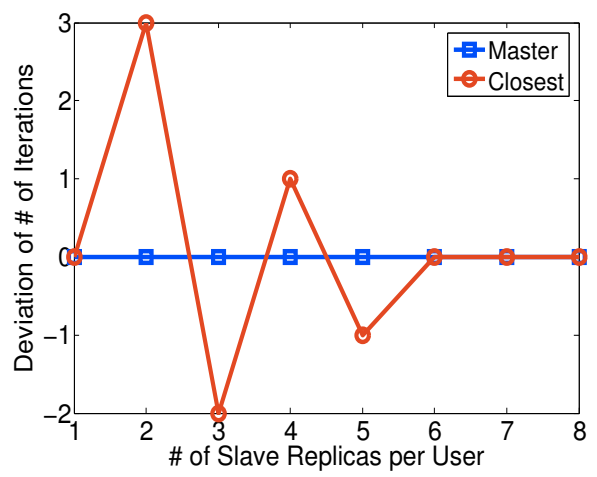

Figure 4.24: Influence of slave placements on iteration \#

How does the initial placement influence the benefit of our approach? Fig. 4.21 runs our approach using 100 different random placements as the initial placements respectively and focuses on how much more total cost the resultant placements have, compared with running our approach using greedy placement as the initial placement. We see that using random placement in initialization leads to placements with up to $10 \%$ more cost, suggesting greedy placement is a good choice for initialization. Under the master policy, few placements initialized by random exceed the placement initialized by greedy by more than $5 \%$ cost. In other words, compared with handling the closest policy, our approach tends to be less affected by the choices of initial placements (i.e., random or greedy) when handling the master policy. This is possibly because the former depends on the locations of all replicas to identify the closest one while the latter depends more on where the master is.

How does the slave placement influence the benefit of our approach? Fig. 4.22 uses the exhaustive approach to place slaves during our fixed-point iterations and compares the total costs of the resultant placements with those using the greedy approach to place slaves during the iterations, all 
adopting greedy placement in initialization. In contrast to the choices of initial placements as in Fig. 4.21, the choices of placing slaves almost does not influence the resultant cost at all, as the deviations of the cost fluctuates only between $\pm 0.3 \%$. This provides strong support for us not to use the exhaustive approach to place slaves; placing slaves greedily is sufficient. Under the master policy, our approach even achieves exactly the same resultant cost no matter placing slaves greedily or exhaustively in each iteration. The reason is in this case the placement of a user's each slave is independent so that the greedy approach and the exhaustive search lead to the same placements of all of a user's slaves.

How does the initial placement influence the execution of our approach? Fig. 4.23 investigates how many more iterations are executed until convergence when running our approach with random placement as the initial placement, compared with using greedy placement as the initial placement. A negative number means the number of iterations in the former case is actually fewer than in the latter case. According to this figure, the number of iterations under the master policy do not deviate that much compared with the number of iterations under the closest policy. Note that in Fig. 4.19 the closest policy with greedy as the initial placement needs to run 5 iterations, the same policy now with random as the initial placement needs to run up to 9 additional iterations; compared with Fig. 4.21, the cost of the resultant placement is larger even when more iterations are executed. The number of iterations depends on the placements of masters and slaves at each iteration, which is often complex, making it hard to predict; how good the optimization results are does not seem to correlate with the number of iterations executed.

How does the slave placement influence the execution of our approach? Fig. 4.24 shows the number of additional iterations that are executed until convergence when running our approach using exhaustive placement for slaves during each iteration, with greedy placement as the initial placement. Due to the same reason as explained with Fig. 4.22, the number of iterations is the same for either the exhaustive or greedy placement of slaves under the master policy. Under the closest policy, the number of additional iterations fluctuates. Compared with Fig. 4.23, we see that, under both data access policies, the slave placement is less influential on the number of iterations executed compared with the initial placement.

\subsection{Discussions}

\subsubsection{Multi-Cloud Access Policies}

The multi-cloud access policy $z_{u, v}$ matters. The graph cuts technique that we use requires the pairwise cost to obey the regularity property [56]. Translat- 
ed into our case, it means when solving the master replicas placement problem, $V_{u \rightarrow v}\left(\mathbf{m}_{u}, \mathbf{m}_{v}\right)+V_{v \rightarrow u}\left(\mathbf{m}_{v}, \mathbf{m}_{u}\right)$ must satisfy $V_{u \rightarrow v}(i, i)+V_{v \rightarrow u}(i, i)+V_{u \rightarrow v}(j, j)+$ $V_{v \rightarrow u}(j, j) \leq V_{u \rightarrow v}(i, j)+V_{v \rightarrow u}(j, i)+V_{u \rightarrow v}(j, i)+V_{v \rightarrow u}(i, j), \forall u, v, i, j . z_{u, v}$ is an important factor that determines whether and how $V_{u \rightarrow v}\left(\mathbf{m}_{u}, \mathbf{m}_{v}\right)+V_{v \rightarrow u}\left(\mathbf{m}_{v}, \mathbf{m}_{u}\right)$ satisfies regularity. Regularity encourages the co-location of the masters of users who have interactions between them, which matches the requirement of social data placement. In this section, we analyze how our case satisfies regularity.

For the relay mode, to align $V_{u \rightarrow v}+V_{v \rightarrow u}=V_{u \rightarrow v}^{c}+V_{u \rightarrow v}^{d}+V_{u \rightarrow v}^{t}+V_{v \rightarrow u}^{c}+$ $V_{v \rightarrow u}^{d}+V_{v \rightarrow u}^{t}$ with regularity, we deduce two sufficient conditions: $z_{u, v}\left(\mathbf{m}_{u}, \mathbf{m}_{v}\right)=$ $z_{u, v}\left(\mathbf{m}_{u}\right), \forall u, v ; z_{u, v}\left(\mathbf{m}_{u}, \mathbf{m}_{v}\right)=\mathbf{m}_{v}, \forall u, v$. The regularity is satisfied as long as one of these two conditions holds. The former requires $z_{u, v}$, the selection of one of $v$ 's clouds, not depend on which $v$ 's master cloud is. This can be implemented by making the selection only out of $v$ 's slave clouds, e.g., selecting the slave cloud of $v$ that is closest to $u$ 's master cloud. The latter requires $z_{u, v}$ always select $v$ 's master cloud, which is naturally true in a system with no slave replicas.

For the redirect mode, we need to align $V_{u \rightarrow v}+V_{v \rightarrow u}=V_{u \rightarrow v}^{c}+V_{u \rightarrow v}^{d}{ }^{\prime}+$ $V_{v \rightarrow u}^{c}+V_{v \rightarrow u}^{d}{ }^{\prime}$ with regularity. The corresponding two sufficient conditions are $z_{u, v}\left(\mathbf{m}_{u}, \mathbf{m}_{v}\right)=z_{u, v}\left(\mathbf{m}_{u}\right), \forall u, v$ and $z_{u, v}\left(\mathbf{m}_{u}, \mathbf{m}_{v}\right)=z_{u, v}\left(\mathbf{m}_{v}\right), \forall u, v$. The former has been explained above; the latter requires the selection of one of $v$ 's clouds not depend on which $u$ 's master cloud is, which can be implemented by selecting the cloud with $v$ 's replica (either master or slave) that is closest to $u$. Note that, compared with the conditions for the relay mode, $z_{u, v}\left(\mathbf{m}_{u}, \mathbf{m}_{v}\right)=z_{u, v}\left(\mathbf{m}_{v}\right)$ contains $z_{u, v}\left(\mathbf{m}_{u}, \mathbf{m}_{v}\right)=\mathbf{m}_{v}$, i.e., the graph cuts technique covers more access policies in the redirect mode than in the relay mode, which is partially because we consider one more dimension, the inter-cloud dimension, in the latter.

We believe the above conditions are able to cover a range of real-world multicloud access policies. A specific policy that does not meet any of the above conditions does not necessarily mean we cannot apply graph cuts to solve the placement problem. For example, consider the following policy:

$$
z_{u, v}=\left\{\begin{array}{l}
\mathbf{m}_{u}, \text { if } \mathbf{m}_{u}=\mathbf{m}_{v} \text { or } \mathbf{m}_{u}=\mathbf{s}_{v, l}, \exists l \in\{1, \ldots, \mathbf{k}\} \\
z_{u, v}^{\prime}\left(\mathbf{m}_{u}, \mathbf{m}_{v}\right), \text { otherwise }
\end{array},\right.
$$

where $u$ always accesses her master cloud if $v$ has a replica co-located there, and accesses another cloud if not. One can verify that this $z_{u, v}$ does not necessarily satisfy regularity. The approach that we take to handle such cases is that, based on the real-world, specific inputs to the problem, we can often easily tune the weights associated with the objectives in order to make $V_{u \rightarrow v}\left(\mathbf{m}_{u}, \mathbf{m}_{v}\right)+V_{v \rightarrow u}\left(\mathbf{m}_{v}, \mathbf{m}_{u}\right)$ satisfy regularity in value. In the relay mode for example, $V_{u \rightarrow v}(i, i)+V_{v \rightarrow u}(i, i)+V_{u \rightarrow v}(j, j)+V_{v \rightarrow u}(j, j)=\left(\alpha_{i}+\alpha_{j}\right)\left(r_{u v}+r_{v u}\right)$, and $V_{u \rightarrow v}(i, j)+V_{v \rightarrow u}(i, j)+V_{u \rightarrow v}(j, i)+V_{v \rightarrow u}(j, i)=\left(\alpha_{z_{u, v}^{\prime}(i, j)}+\alpha_{z_{u, v}^{\prime}(j, i)}\right) r_{u v}+$ $\left(\alpha_{z_{v, u}^{\prime}(i, j)}+\alpha_{z_{v, u}^{\prime}(j, i)}\right) r_{v u}+\left(V_{u \rightarrow v}^{d}(i, j)+V_{u \rightarrow v}^{t}(i, j)+V_{u \rightarrow v}^{d}(j, i)+V_{u \rightarrow v}^{t}(j, i)+\right.$ 
$\left.V_{v \rightarrow u}^{d}(i, j)+V_{v \rightarrow u}^{t}(i, j)+V_{v \rightarrow u}^{d}(j, i)+V_{v \rightarrow u}^{t}(j, i)\right)$. It is obvious that the the latter equation is definitely no smaller than the former if $\alpha_{i}=\alpha_{j}, \forall i, j$ holds. As discussed in Section 4.2.6, $\alpha_{i}$ 's can capture the carbon intensity, the electricity or VM price. In reality, the difference of each of these quantities at different clouds or regions does not differ by more than one order of magnitude [40, 74] — it is thus easy to tune the weights associated with $V_{u \rightarrow v}^{d}, V_{u \rightarrow v}^{t}$ and $\alpha_{i}$ to make the latter equation large enough, which translates into the practical success of graph cuts no matter what $z_{u, v}$ is used by service providers.

\subsubsection{Optimality and Scalability}

We briefly discuss our perspectives on how optimal the solutions found by our approach can be and how our algorithm can scale to a huge user population.

Optimality: We believe our solutions are reasonably good, as the two subproblems are either solved by the current state-of-the-art technique of graph cuts or fairly easy to be solved due to the independence among users. To the best of our knowledge, each subproblem is solved to the best that can be achieved to date. Although our approach may not find the Pareto efficient solution, the advantages of our solutions are experimentally justified by our evaluations with real-world data which will be shown in the next section.

Scalability: The most time-consuming part of our approach is calculating the minimal $s$ - $t$ cut by the max-flow algorithm, especially for an extremely large user population. However, in socially aware services, users often form communities within which they interact heavily and across which sparsely. We can thus partition the user base into communities by algorithms like METIS [53], and then apply our approach to each community independently. We will also demonstrate some results regarding this point.

\subsection{Summary}

While socially aware services attract billions of users, the need for such services to meet multiple system objectives has become compelling. The unique features of socially aware services that distinguish themselves from other Internet services pose a new problem of optimizing data placement over multiple geographically distributed clouds.

In this chapter, we firstly build models that generalize to a variety of system objectives, capturing user interactions, the master-slave paradigm, and the multi-cloud access policies. We then propose an approach with multiple iterations, with each iteration solving master and slave data placement separately, leveraging our finding that the master placement subproblem can be effectively solved via graph cuts. Evaluations with real-world data show that our approach 
is not only able to optimize every dimension of the socially aware service, but can also pursue a diversity of trade-offs among objectives, converge fast, and scale to a large user base; they further show that different choices of the initial placement of all replicas and different methods of slave replicas placement influence the optimization results and the algorithm performance to different extents, shedding light on how to better control our algorithm to achieve desired optimizations. 



\section{Chapter 5}

\section{OSN Data Placement in Data Center for Network Performance}

\subsection{Introduction}

Online Social Networks (OSNs) are extremely popular destinations for Internet users nowadays. With users of such a huge scale, it is imperative to implement a scalable backend system to support users' data storage and access. Current OSN data center infrastructures often adopt distributed DBMS (e.g., MySQL) and/or key-value stores (e.g., Cassandra [57]), which essentially distribute users' data among servers randomly. Though simple and efficient, random distribution fails to match the OSN data access patterns and can suffer from performance problems. For instance, in a typical OSN service such as Facebook News Feed or Twitter, to display a user's home page, the service must access and collect the data of this user's every friend from multiple servers, with unpredictable response time determined by the server with the highest latency. This problem is quite severe when servers and the data center network are under heavy workload.

To address this issue, it has been proposed to replicate the data of a user's every friend to the server where this user's own data are stored, i.e., maintaining social locality so that services such as News Feed can be resolved within a single server [72]. In this paradigm, each user has a single master replica, with which the replicas of friends' data are co-located on the same server; each user also has multiple slave replicas on different servers co-located with friends' master replicas. The overheads of social locality are two-fold: the replication storage of slave replicas and the traffic from master to slaves to maintain consistency.

Existing work mainly focuses on optimizing the storage [72,81], yet overlooks the traffic aspect. According to [72], the minimum average number of slave replicas per user to ensure social locality is up to 20 for an OSN service on a cluster of 512 servers. Given about 3.2 billion daily Facebook comments [5] and the average packet size of $1 \mathrm{~KB}$, the traffic for synchronizing replicas can be up to about 60 TB per day, which could consume considerable data center network resources, not to mention other user-generated contents. In industrial 
data centers, the networks are often the bottleneck [14, 62, 75]; besides, the user-facing service traffic (e.g., the traffic between OSN service and OSN users) and the backend synchronization traffic shares a common data center network infrastructure, competing for network resources [11]. Therefore, optimizing the backend traffic can yield more network resources for the user-facing service, and can improve the salability of data center networks.

In this chapter, we study the problem of social-locality-aware partitioning of the OSN data backend in a data center environment. While embracing social locality's advantages such as eliminating unpredictable inter-server response time, we aim to minimize its overhead in the traffic aspect without ruining the existing optimization (if any) of the storage aspect.

We explicitly consider data center network topologies (e.g., tree [11, 62, 87], Clos topology $[14,15,43]$, etc.) together with social locality. Different topologies have different features, we thus define diverse network performance goals for the synchronization traffic to save network resource consumption. We further formulate the traffic optimization problem and propose a unified solution to achieve all network performance goals - our traffic-aware partitioning algorithm which is inspired by the fact that carefully swapping the roles of the master replica and a slave replica of a user can lead to traffic reduction. Trace-driven simulations with a large-scale, real-world Twitter dataset demonstrate that, compared with state-of-the-art algorithms, such as random placement (i.e., the standard placement in MySQL and Cassandra), SPAR [72], and METIS [53], our algorithm can reduce the synchronization traffic by approximately $30 \%-70 \%$ in a variety of data center network topologies with a number of servers, without affecting the existing load balance among servers and increasing the total replication storage.

The rest of this chapter is organized as follows. Section 5.2 introduces social locality, data center networks, and proposes network performance goals. Section 5.3 presents the problem formulation. Section 5.4 elaborates our trafficaware partitioning algorithm. Section 5.5 describes our experiments and shows the evaluation results. Section 5.6 analyzes the complexity of our algorithm and discusses the design trade-off. Section 5.7 concludes.

\subsection{Models}

We briefly introduce the social locality paradigm and data center network topologies. For different topologies, we propose diverse network performance goals for the synchronization traffic. We then present the traffic optimization problem with diverse goals by a unified formulation. 


\subsubsection{Revisiting Social Locality}

"Social locality" is a data replication scheme independent of data partitioning. The former means how many replicas should a piece of data have and on which sever to place each replica, while the latter means how to divide the whole dataset into separate subsets in order to place each of them on a different server. The social locality scheme chooses to replicate the data of a user's every friend on the server that hosts this user's own data, which has proved to be an effective approach to overcome the performance problems of OSN services.

Social locality is a single-master-multi-slave paradigm. The partition that hosts a user's master is determined by the partitioning scheme; the partitions that host a user's slaves are determined by the social relations among users. Replica consistency is maintained by the synchronization traffic from a user's master to her slaves. Load balance in this context refers to balancing the number of masters among servers [72].

\subsubsection{Encoding Network Performance Goals}

The de facto standard of data center network topology is the two- or three-layer tree [11], interconnecting servers by switches and/or routers. In a three-layer tree, at the bottom, servers in the same rack are connected to a top-of-rack or edge switch. Each edge switch is connected to an aggregation switch, and each aggregation switch is connected to one or multiple core switches. Given that each edge switch connects with $k_{1}$ servers and each aggregation switch connects with $k_{2}$ edge switches, the number of servers that are hosted by one aggregation switch is thus $k_{1} k_{2}$. The tree topology is quite often oversubscribed in modern data centers in order to lower the total cost of such design [14].

A couple of full-capacity topologies (e.g., fat-tree [14], BCube [41], etc.) have been proposed to overcome the oversubscription problem of the tree topology. Fat-tree is a design that is composed of servers and $k$-port switches. In a fattree, there are $k$ pods with $k / 2$ edge switches and $k / 2$ aggregation switches in each pod, $k^{2} / 4$ core switches, and $k^{3} / 4$ servers. The $k / 2$ ports of each edge switch connect with $k / 2$ servers, and the rest $k / 2$ ports connect with different aggregation switches in the same pod. The rest $k / 2$ ports of each aggregation switch connect with different core switches.

Fig. 5.1 depicts a tree and a fat-tree, with $k_{1}=k_{2}=2$ and $k=4$, respectively.

Targeting at different data center topologies, we define diverse performance goals for the synchronization traffic. For the tree topology, it is desirable to localize the traffic to save the utilization of the oversubscribed upper-layer network links [75]. If a user's master and all her slaves are on servers in the same rack, synchronization only involves intra-rack traffic; otherwise, the synchronization traffic must go beyond the edge switch to upper layers in order to reach replicas 


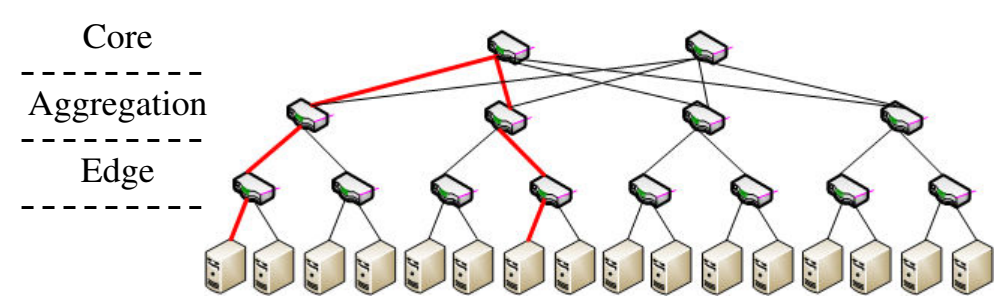

(a) Three-layer tree

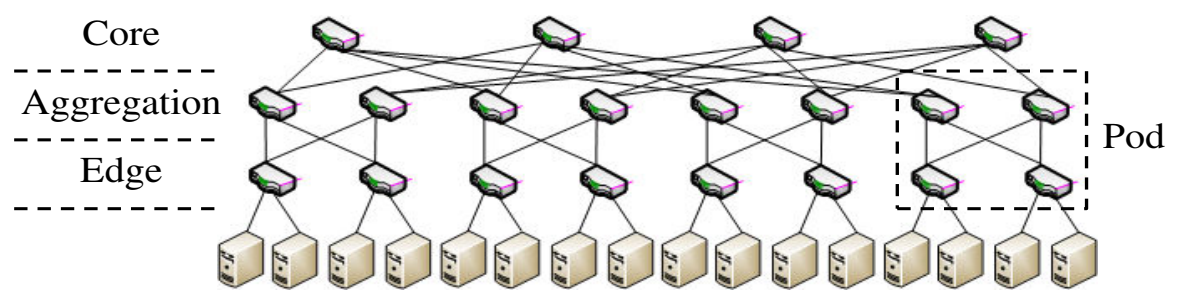

(b) Fat-tree

Figure 5.1: Data center network topologies

on servers in another rack. Fig. 5.1(a) uses red lines to exemplify a path between servers via the core switch. We consequently define the following goal of traffic localization:

- Goal \#1 Minimize the core synchronization traffic, i.e., the traffic traveling through core switch(es)

For full-capacity topologies, it is not necessary to localize the traffic due to the absence of oversubscription [75], but it is desirable to reduce the utilization of every switch and link to improve the network scalability [62]. We thus have the following goal, which is also applicable to the tree topology:

- Goal \#2 Minimize the total synchronization traffic, i.e., the sum of the traffic perceived by every switch

Note that the performance goals of a data center network are not limited to the ones that we define here, e.g., for a fat-tree, localizing the traffic also makes sense if upper-layer links suffer from congestion. As will be shown next, it is easy to use our model to express any network performance goal, and our proposed algorithm provides a general and efficient approach to reach all these goals.

\subsection{Problem}

OSN is often modeled as a graph [64], where each user is represented by a vertex and each social relation between two users is represented by an edge between 
the corresponding two vertices. We additionally consider each user's traffic rate (i.e., the size of the data generated by a user).

Given such a social graph with each user's traffic rate, we are interested in the problem of partitioning the graph into $N$ partitions, maintaining social locality for each user with the synchronization traffic achieving our network performance goals. Additional inputs to the problem include pre-specified numbers of master replicas on each server and a pre-specified total number of slave replicas in the entire system. Our partitioning ensures that, the number of masters on each server equals to the corresponding pre-defined number for this server (i.e., guaranteeing the load assignment across servers, or load balance if such number is the same for all servers), and the total number of slaves in the system does not exceed the pre-defined number (i.e., guaranteeing the total replication storage within the given quota).

We introduce notations to formulate the problem. $G=(V, E)$ denotes the undirected social graph, where $V$ is the set of vertices (i.e., users) and $E$ is the set of edges (i.e., social relations). $e_{i j}$ is the edge between user $i$ and user $j$. $t_{i}$ is the traffic rate of user $i . A(i, j)$ is the value representing the adjacency between server $i$ and server $j$ in the $N \times N$ control matrix $A$, where $N$ is the total number of servers. $M_{j}$ is the pre-defined number of masters on server $j$ and $S$ is the pre-defined total number of slaves in the system. $m(i, j)$ is binary, being 1 if the master of user $i$ is assigned to server $j$ and being 0 otherwise. Thus $m_{i}=\sum_{\forall j}(j \times m(i, j))$ is the server which hosts user $i$ 's master. $s(i, j)$ is similar to $m(i, j)$ but representing the assignment of salves to servers. We formulate the problem as follows.

\section{minimize}

$$
\sum_{\forall i} \sum_{\forall j \in\left\{j \mid s_{i j}=1, \forall j\right\}}\left(t_{i} \times A\left(m_{i}, j \times s(i, j)\right)\right)
$$

\section{subject to}

$$
\begin{gathered}
\sum_{\forall j} m(i, j)=1, \forall i \\
m\left(i^{\prime}, m_{i}\right)+s\left(i^{\prime}, m_{i}\right)=1, \forall i, i^{\prime}, e_{i i^{\prime}} \in E \\
\sum_{\forall i} m(i, j)=M_{j}, \forall j \\
\sum_{\forall i} \sum_{\forall j} s(i, j) \leq S
\end{gathered}
$$

The objective is to minimize the traffic from masters to slaves, counted by a given control matrix. Each of our goals can be expressed by a particular control matrix. Thus our problem formulation applies uniformly to all the goals 
that are defined previously. Constraint (5.1) ensures a single master for every user. Constraint (5.2) ensures social locality for every user. Constraint (5.3) ensures that the distribution of masters on servers matches the pre-defined load assignment. Constraint (5.4) ensures that the total replication storage does not exceed the pre-defined quota.

The control matrix is used to count the traffic for a given performance goal in a given data center topology. For Goal \#1, we only care about the core-layer traffic, and thus we only set the adjacency value between any two servers located under different aggregation switches to 1 . For Goal \#2, we count the traffic at every switch. If there are $n$ switches in the communication path between any two servers, the adjacency value between these two servers are set to $n$. Aligned with the descriptions of data center network topologies in Section 5.2.2, we present as follows the control matrices for the goals of the tree and the fat-tree topology, respectively.

- Control matrix of tree for Goal \#1:

$$
A_{t 1}(i, j)=\left\{\begin{array}{l}
0, i=j \\
0, i \neq j \wedge\left\lfloor\frac{i}{k_{1}}\right\rfloor=\left\lfloor\frac{j}{k_{1}}\right\rfloor \\
\left.0, i \neq j \wedge\left\lfloor\frac{i}{k_{1}}\right\rfloor \neq\left\lfloor\frac{j}{k_{1}}\right\rfloor\right\rfloor\left\lfloor\frac{i}{k_{1} k_{2}}\right\rfloor=\left\lfloor\frac{j}{k_{1} k_{2}}\right\rfloor \\
1, \text { otherwise }
\end{array}\right.
$$

- Control matrices of tree and fat-tree for Goal \#2:

$$
\begin{aligned}
& A_{t 2}(i, j)=\left\{\begin{array}{l}
0, i=j \\
1, i \neq j \wedge\left\lfloor\frac{i}{k_{1}}\right\rfloor=\left\lfloor\frac{j}{k_{1}}\right\rfloor \\
\left.3, i \neq j \wedge\left\lfloor\frac{i}{k_{1}}\right\rfloor \neq\left\lfloor\frac{j}{k_{1}}\right\rfloor\right\rfloor\left\lfloor\frac{i}{k_{1} k_{2}}\right\rfloor=\left\lfloor\frac{j}{k_{1} k_{2}}\right\rfloor \\
5, \text { otherwise }
\end{array}\right. \\
& A_{f t 2}(i, j)=\left\{\begin{array}{l}
0, i=j \\
1, i \neq j \wedge\left\lfloor\frac{2 i}{k}\right\rfloor=\left\lfloor\frac{2 j}{k}\right\rfloor \\
3, i \neq j \wedge\left\lfloor\frac{2 i}{k}\right\rfloor \neq\left\lfloor\frac{2 j}{k}\right\rfloor\left\lfloor\left\lfloor\frac{4 i}{k^{2}}\right\rfloor=\left\lfloor\frac{4 j}{k^{2}}\right\rfloor\right. \\
5, \text { otherwise }
\end{array}\right.
\end{aligned}
$$

\subsection{Algorithm}

The traffic optimization problem is an Integer Linear Program that generally belongs to NP-hard problems. We thus focus on developing a heuristic approach that works well in practice.

\subsubsection{Achieving Performance Goals via Role-Swaps}

Starting with an initial solution (i.e., a trial assignment of all replicas to servers), our algorithm tweaks this solution iteratively to search the solution space. Each 
tweak operation changes the current assignment into a new one that has less traffic counted by the control matrix, without violating any constraint.

Swapping the roles of a user's master and slave can be used as the tweak operation. As an example, Fig. 5.2 is a local view of part of the tree topology, where three servers are interconnected by two edge switches and one aggregation switch. The box below each server shows the current data replicas on this server. There are four users $u, v_{1}, v_{2}$ and $v_{3}$. Black circles are masters, and red ones are slaves that exist for maintaining social locality of masters. Solid lines represent social relations and dotted arrows represent the synchronization traffic. Let's consider the total traffic perceived by every switch, and let's assume all users have the same traffic rate of 1 unit, for example. The existing data assignment has the total traffic of 15 units, as in Fig. 5.2(a). Fig. 5.2(b) and Fig. 5.2(c) perform the role-swaps. Firstly, we swap the roles of user $u$ 's master and her slave $u^{\prime}$, reducing the total traffic to 11 units. Secondly, we select $v_{2}$ and $v_{2}^{\prime}$, and swap the roles in order to maintain the existing load assignment. Social locality must be maintained after each role-swap. Overall, we achieve 4 units traffic reduction without altering the load assignment of the existing data placement (i.e., the number of masters on each server remains the same before and after the two role-swaps) and without increasing the total replication storage (i.e., the total number of slaves does not increase after the two role-swaps).

Note that, in order to always guarantee the load assignment, a single tweak must include two role-swaps, i.e., we must select two users and do the role-swap for each of them. The two users' masters are on different servers, and each user's slave is co-located with the other user's master. Only two users satisfying this condition can be considered as candidates for a tweak operation.

\subsubsection{Traffic-Aware Partitioning Algorithm}

Algorithm 6 provides the pseudo codes of our partitioning algorithm. $p_{\text {start }}$ is the starting solution; Best maintains the best solution that has been found. For a tweak, two users $u$ and $v$ are selected. $m_{u}$ is the server that hosts $u$ 's single master, and $s_{u}$ is the server that hosts $u$ 's slave involved in this tweak. $\mu_{u}$ is the total number of slave replicas that can be reduced and $\tau_{u}$ is the amount of traffic (counted by the control matrix) that can be saved by swapping the roles of $u$ 's master on $m_{u}$ and her slave on $s_{u} . m_{v}, s_{v}, \mu_{v}$, and $\tau_{v}$ have similar meanings for user $v . \Delta$ denotes the total number of slave replicas that has been reduced so far compared with the starting solution.

Algorithm 6 adopts a hill-climbing strategy by requiring that every tweak must reduce the traffic (i.e., $\tau_{u}+\tau_{v}>0$ ). We are aware of other design options, e.g., Simulated Annealing [55] which in our case allows tweaks with traffic increase, etc. While such techniques may discover better solutions or approximate 


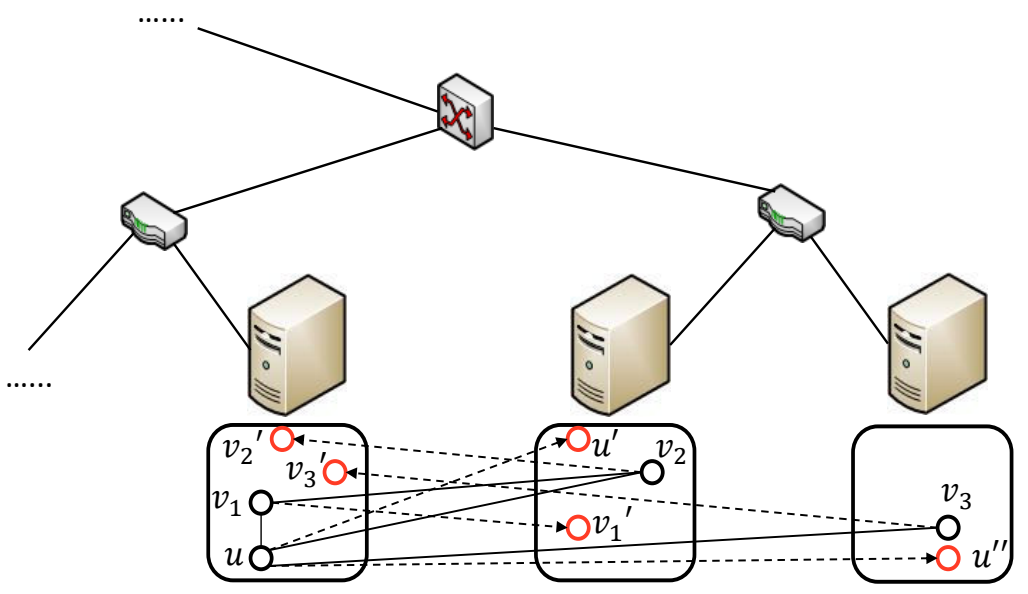

(a) Before swapping: traffic $=15$

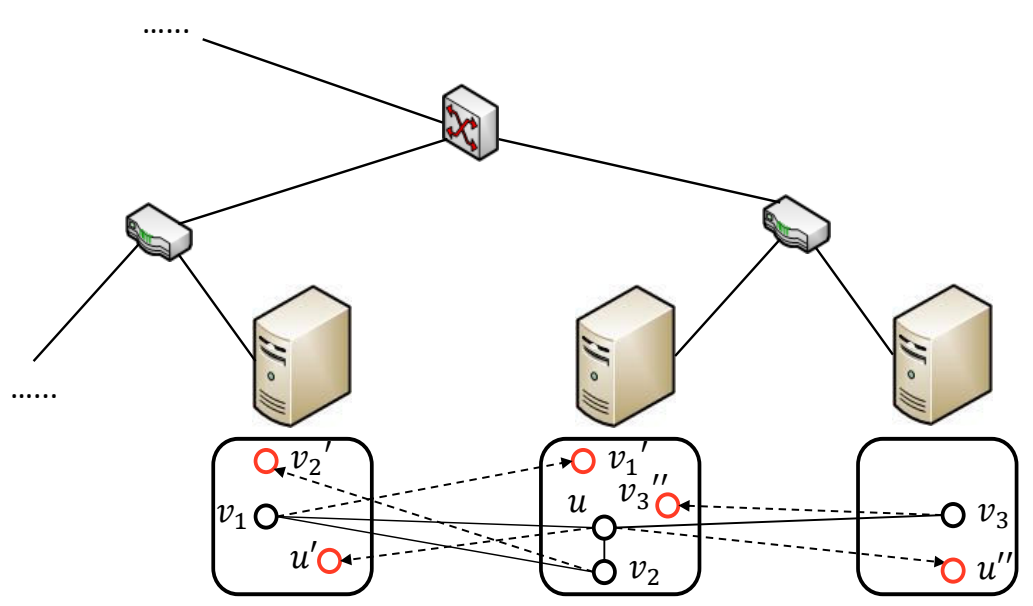

(b) After swapping the roles of $u$ and $u^{\prime}$ : traffic $=11$

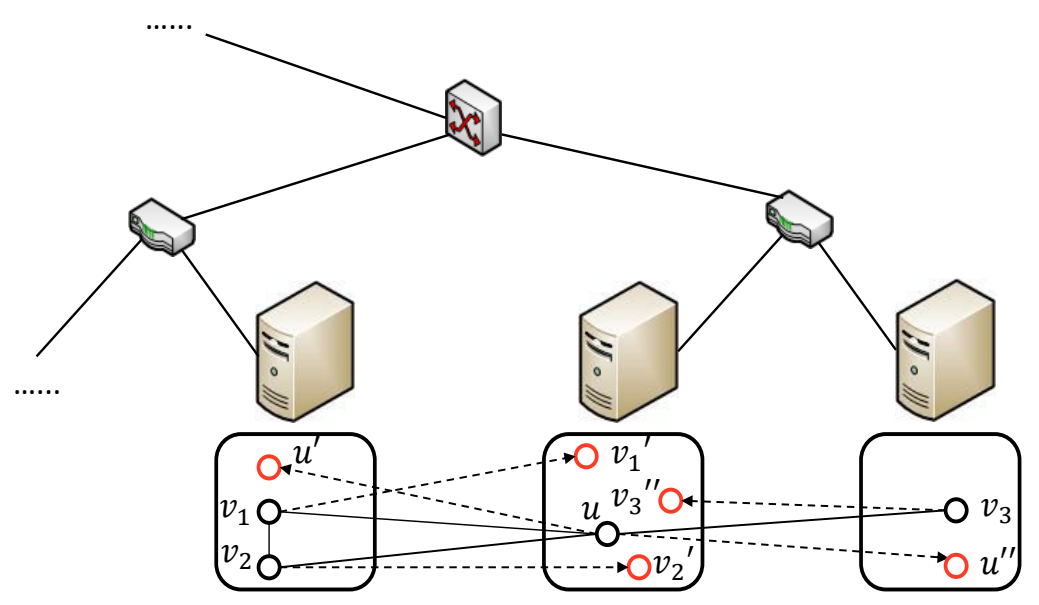

(c) After swapping the roles of $v_{2}$ and $v_{2}^{\prime}$ : traffic $=11$

Figure 5.2: Using role-swaps to reduce the traffic 


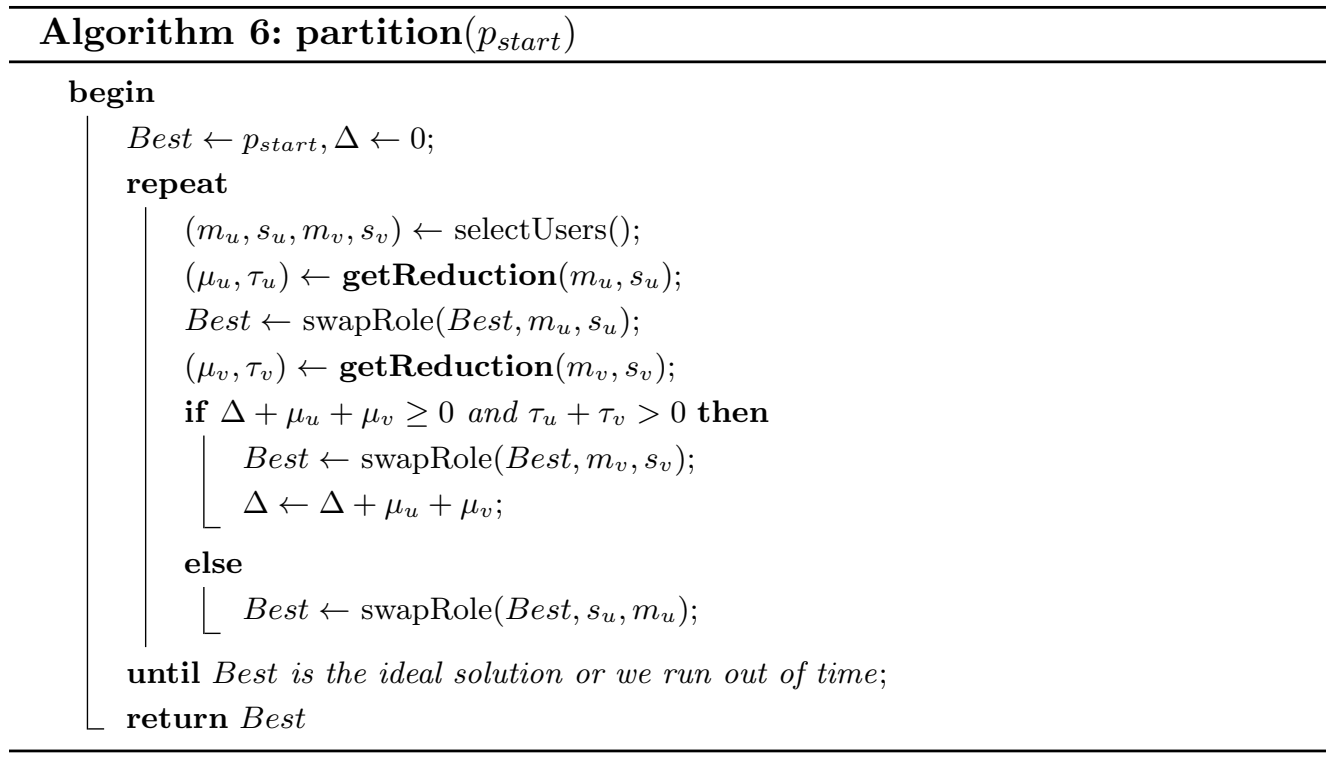

closer to the theoretical optimum(s), it is easy to integrate them to our algorith$\mathrm{m}$. We find that hill-climbing can already achieve significant traffic reductions in practice, as will be shown in Section 5.5.2.

Algorithm 7, invoked by Algorithm 6, specifies the calculation of the replica number reduction and the traffic reduction that can be achieved by a given roleswap. An intuitive alternative to get the reductions is calculating the total replica number and the total traffic before and after a tweak, respectively, and then calculating the difference for each of them. However, compared with Algorithm 7 which only accesses the neighborhood of the selected user, this intuitive approach needs to access every user in the system and can cause considerable computation overhead for a large social graph.

\subsection{Evaluations}

\subsubsection{Experimental Settings}

OSN dataset: By crawling Twitter in a breadth-first searching manner in March 2010, we collected a dataset of 107,734 users with 2,744,006 social relations. For each crawled user, we have her profile, tweets, and the followers list. We use the total size of each user's tweets published in February 2010 as the user's traffic rate.

Data center network topology: We simulate switches of 8,12 , and 16 ports, respectively, and use them to organize a tree and a fat-tree topology. In a fat-tree, the total number of switches and that of servers are determined by the number of ports per switch, as mentioned in Section 5.2.2; in a tree, we always use 2 switches in the core layer. Table 5.1 contains the details of the network 


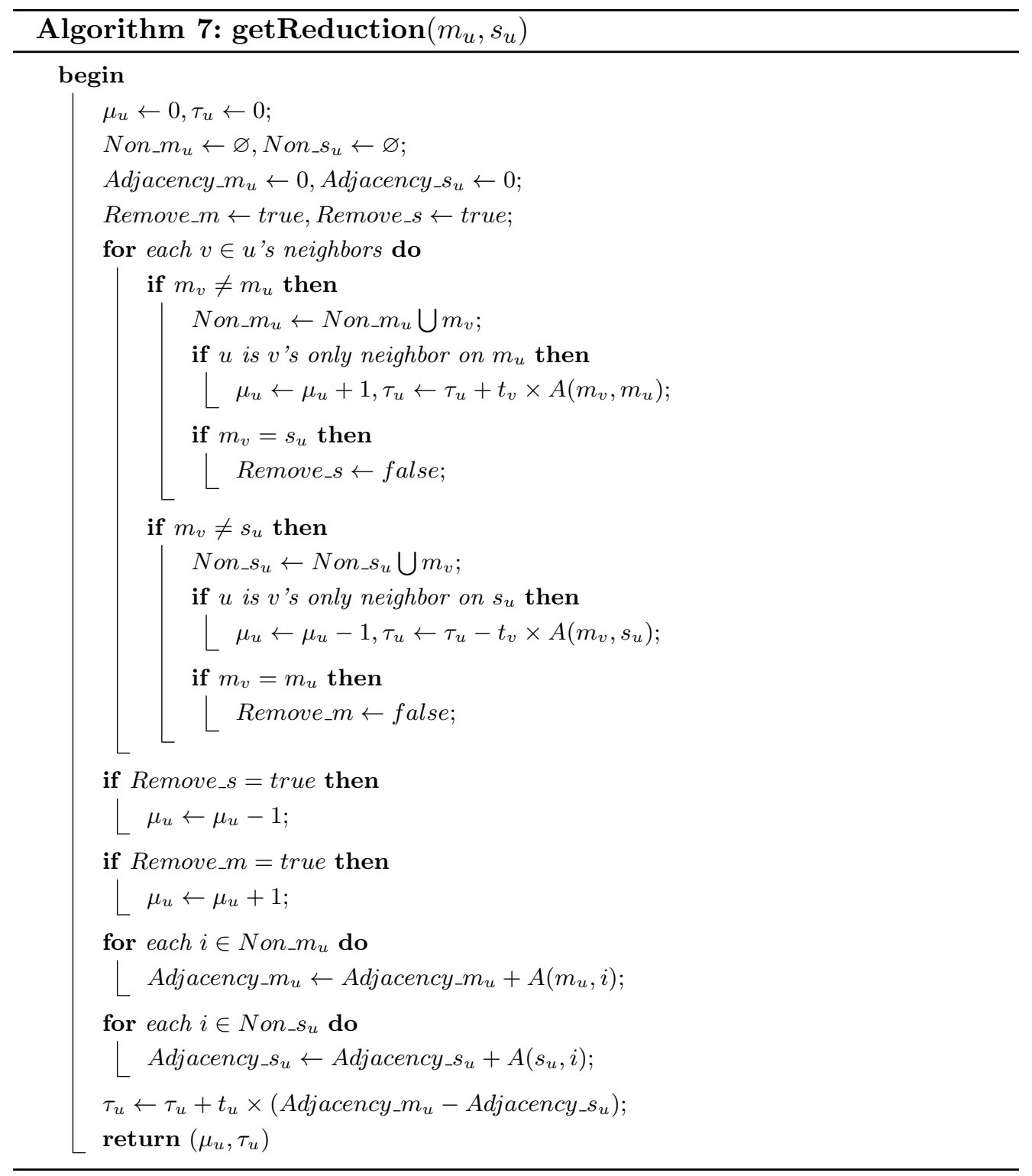

configurations. For each topology, we build the corresponding control matrix by the formulas presented in Section 5.3.

Initial assignment: The initial assignment is the starting solution in our evaluations. For our social graph with each user's traffic rate, we use random placement, SPAR [72], and METIS [53] to generate an initial assignment of master replicas to servers, respectively; slave replicas are then assigned to servers to maintain social locality for each user. We implement SPAR, strictly following [72]; METIS has an open-source implementation that we can directly use.

\subsubsection{Evaluation Results}

The results are illustrated in Fig. 5.3, 5.4, 5.5, and 5.6, where "TA-" denotes our traffic-aware partitioning, starting with a specified initial assignment, and traffic 
Table 5.1: Data center network configurations

\begin{tabular}{|c|l||c|c|c|}
\hline \multicolumn{2}{|c||}{$k, k_{1}, k_{2}$} & 8 & 12 & 16 \\
\hline \multicolumn{2}{|c|}{ \# of servers } & 128 & 432 & 1024 \\
\hline \multirow{2}{*}{ \# of switches } & tree & 20 & 41 & 70 \\
\cline { 2 - 5 } & fat-tree & 80 & 180 & 320 \\
\hline
\end{tabular}

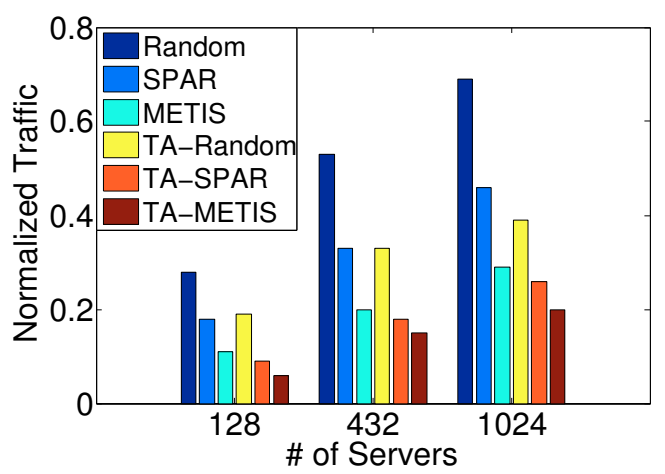

Figure 5.3: Core-layer traffic

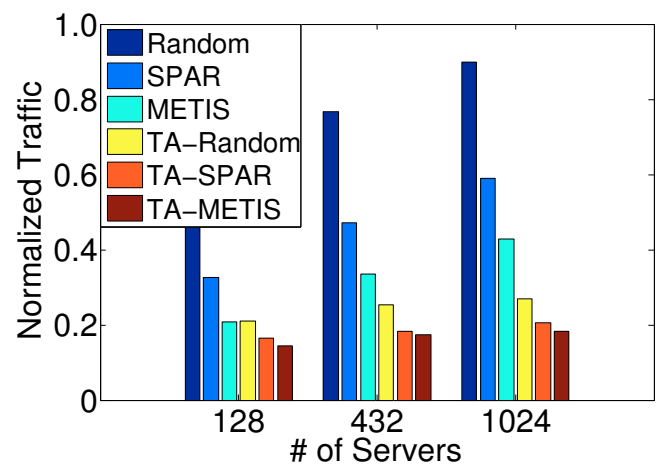

Figure 5.5: Perceived traffic (fat-tree)

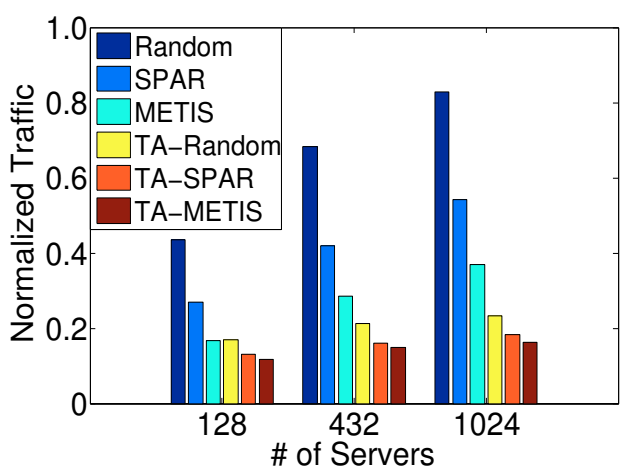

Figure 5.4: Perceived traffic (tree)

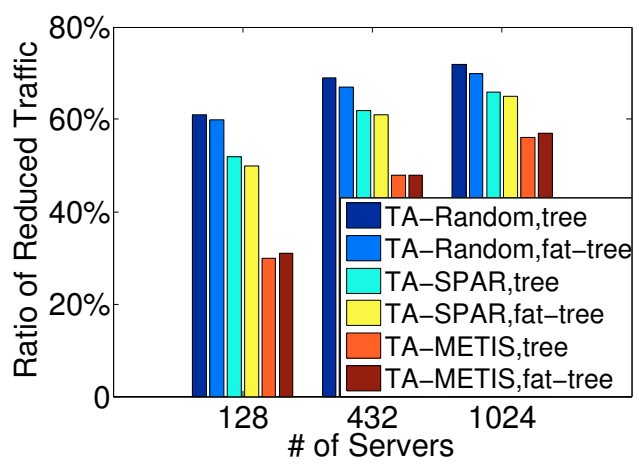

Figure 5.6: Traffic reduction ratio

values have been normalized.

Fig. 5.3 shows the traffic that passes the core-layer switches in the tree topology. We use our algorithm to reduce such core-layer traffic to achieve Goal \#1. Fig. 5.4 and 5.5 compare the total traffic perceived by every switch in a tree and a fat-tree, respectively. We apply our algorithm to reduce such traffic to achieve Goal \#2. We find that, for any given topology with a fixed number of servers, users placed by METIS tend to incur less core-layer traffic and total perceived traffic than by random and SPAR. This meets our expectation. METIS explicitly minimizes the inter-server traffic, while SPAR minimizes the total number of slave replicas, equivalent to minimizing the inter-server traffic with the assumption that each user has the same traffic rate, i.e., SPAR essentially ignores the difference of users' traffic rates. As the number of servers increases, the number 
of users per server drops and users tend to be placed under different aggregation switches, causing the core-layer traffic and the total perceived traffic to grow.

We notice that our traffic-aware partitioning algorithm can significantly reduce the core-layer traffic and the total perceived traffic on top of random, SPAR and METIS, respectively. Our algorithm makes no assumption about the initial assignment and the underlying data center network topology, and can work effectively. Fig 5.6 makes clear the ratio of the reduced traffic over the total perceived traffic. It is easy to see that our algorithm is not sensitive to the type of data center topologies since tree and fat-tree have similar traffic reduction ratios for a fixed number of servers. It also shows that random has the largest traffic reduction ratio than SPAR and METIS, implying that random has a larger room for our algorithm to optimize. Both SPAR, which optimizes the total replication storage, and METIS, which optimizes the inter-server traffic, cannot automatically meet our Goal \#1 or Goal \#2 (i.e., they do not place replicas to achieve specific network performance goals), while our traffic-aware partitioning algorithm can minimize the traffic to achieve the network performance goals.

\subsection{Discussions}

We discuss the complexity of our algorithm, which partially guides its design. The time complexity of Algorithm 7 is $O(|V|+2 \times N)=O(|V|)$, where $|V|$ is the total number of users and $N$ is the total number of servers, given $N \ll$ $|V|$. Without Algorithm 7, the intuitive method of calculating the reductions as mentioned above will be of $O\left(|V|^{2}\right)$.

In Algorithm 6, we need to select two users with their role-swaps. Different selection strategies usually have different trade-offs between time complexity and the amount of traffic reduction. A greedy selection may have a good traffic reduction, but it takes more time, because after a role-swap is performed, we must re-calculate the reductions of role-swaps of all this selected user's neighbors, which takes $O\left(|V|^{2}\right)$ if we do all calculations by Algorithm 7, and then sort all the role-swaps again, which takes $O(|E| \log |E|)$ where $|E|$ is the total number of social relations. Therefore, the greedy selection has a complexity of $O\left(|V|^{2}+\right.$ $|E| \log |E|)$. In contrast, a random selection only has $O(1)$, but may achieve less reductions than greedy. We take random selection here.

\subsection{Summary}

As the OSN data have to be partitioned among multiple servers in a data center, in this chapter we studied how to partition them not only with social locality, but also with optimized inter-server traffic. We model the problem, propose a traffic-aware partitioning algorithm, and evaluate how our algorithm works with 
real-world Twitter data. Our experiments show that regardless of the data center topology and the initial data assignment, our algorithm can significantly reduce the core-layer traffic and the total traffic that is perceived by every switch. Its performance is better than not only random data partitioning, but also state-ofthe-art algorithms including SPAR and METIS. 



\section{Chapter 6}

\section{Conclusion}

\subsection{Comparative Summary}

This thesis selectively studies three different problem settings that we believe are typical to represent real-world scenarios of placing OSN data across clouds and across servers inside a cloud. We model optimization problems with various system objectives, and based on the features of each problem, we propose novel algorithms that find good data placements. Experiments using real-world data traces demonstrate the advantages of our algorithms, compared with other stateof-the-art, de facto, and baseline methods. We also have discussions on algorithm complexity, optimality, scalability, design alternatives, etc.

On one hand, our studies in Chapters 3, 4, and 5 connect with one another.

Chapters 3 and 4 focus on the wide-area multi-cloud scenario, and Chapter 5 focuses on the local-area scenario inside a single cloud. They are orthogonal: if an OSN provider wants to deploy its service at multiple clouds, it may firstly use our work in Chapters 3 and 4 to assign data to clouds, and afterwards for the data assigned to each cloud, it may leverage our work in Chapter 5 to assign them to servers inside a cloud. When placing data across clouds, we assume that each cloud hosts at most one replica for a user, either a master or a slave; however, once such decisions have been made-e.g., if we determine to place a slave replica of a specific user at a specific cloud, then inside this cloud, there can be multiple copies of this slave replica and one of them may be regarded as "master" to synchronize the rest copies for consistency - our work on data placement inside a cloud can be applicable.

On the other hand, our studies in Chapters 3, 4, and 5 differ from one another.

In the problem space, Chapters 3 and 5 assume social locality for every user, where a user has one master and the number of her slaves depends on where her neighbors' masters are placed as her slaves serve the social locality for her every neighbor. This approach guarantees for every user that no read operation needs to go across cloud or server boundaries. The decision variables are actually only the locations of masters, because once masters are placed, the locations of the slaves for social locality are also determined. In contrast, Chapter 4 assumes no social locality but a single master and a fixed number of slaves for every user. A user's every replica, either a master or a slave, has the freedom to be placed at any cloud in the system. Every replica has a corresponding decision variable. 
This approach, while permitting operations to go across clouds, aims to optimize objectives that depend on all operations including reads, writes, and propagated writes to maintain consistency.

Besides, our work in Chapters 3 and 5 uses a coarse-grained manner, assuming some cost associated with every user, and does not explicitly define how the cost of a user depends on the read and write operations performed; in Chapter 4, we make it fine-grained, and relate various system objectives with the number of read and write operations. We model constrained, single-objective optimization problems in Chapters 3 and 5 and model an unconstrained, multi-objective optimization problem in Chapter 4.

In the solution space, Chapters 3 and 5 use the same algorithmic idea about swapping the roles of replicas while achieving various goals during role-swaps: QoS and data availability as in the former and data center traffic performance as in the latter. Role-swap is simply a kind of "tweak" operation, by which, starting with the initial solution, a placement as in our case, one can tweak the solution and check whether the objective, the cost as in our case, becomes better. As such, one tweaks the solution to be better and better in order to reach the optimum gradually. However, it is not straightforward to calculate the objective value of a tweaked solution, and thus we propose the role-swap algorithms so that one can obtain it efficiently in terms of time complexity. Chapter 4 , on the other hand, takes a different approach to solve its problem. Our observations of the problem formulation motivate us to firstly decompose the problem into subproblems and then solve each subproblem given the solution of the other subproblem. By iterating this decomposition procedure for multiple rounds until each subproblem cannot be solved better, we reach a good solution to the original problem. The nature of this approach is that, for an initial solution, we tweak part of it to make the objective better given the other part of it, and do this alternately in multiple rounds to reach the optimum gradually. Our work here focuses on the decomposition and on solving the master replica placement by a special technique, i.e., graph cuts.

Further, in the evaluations, Chapters 3 and 5 use pure Twitter data, including the social graph and the size of each user's tweets, while Chapter 4 uses the Twitter social graph but synthesizes the interactions, i.e., read and write operations among users, to align with the user interaction features disclosed by existing literatures. Chapters 3 and 4 also use users' real-world geographic locations in addition to cloud locations, while Chapter 5 produces data center network topologies as part of inputs. There are many parameters of the inputs to our evaluations. In Chapter 3 where we have a constrained optimization problem, we vary the settings of the constraints to see how the objective is influenced; in Chapter 4 where we have no constraints, we vary the settings to change the inputs to see their influence on the various objectives. 


\subsection{Future Work}

To end this thesis, we share some of our thoughts on the future work.

\subsubsection{Data Placement vs. Request Distribution}

This thesis focuses on data placement and relates various system objectives to the locations of data; however, system objectives can additionally depend on which clouds users' requests are assigned to [40, 58, 88, 95]. Recalling that in Chapter 4 , in the scenario of multiple clouds, we assume that a user always connects to the cloud that hosts her master replica, and her every request, no matter read or write, goes to her master cloud first. In other words, the location of the master replica determines where a user's requests go. Through such a binding, system objectives are modeled as only depending on data locations. But in fact, without such a binding, for any given data placement, optimally assigning users' requests to the clouds that have the required data is yet another optimization problem. For instance, if we permit a user's read request to her own data to be served by any of the clouds that host either a master or a slave replica of hers, then we have a joint optimization problem where we need to determine which clouds to host a user's data replicas as well as which of such clouds is supposed to serve her read requests. We also need to determine, for the read requests to her every friend, which of her friend's clouds that host her friend's data to serve such requests. Note that the write requests, either to her own data or her friend, can still be assumed to be always served by the master cloud of hers or her friend. A more complicated setting can be that we do not use the master-slave paradigm at all, and assume every replica of a user is equal and can receive both read and write operations while write conflicts are handled by other techniques and are out of the scope. In this case, in addition, we need to determine where to assign write requests. Compared with our current version of the data placement problem, the joint optimization problem should be harder, simply because the latter has more decision variables. The optimal solutions of the latter should be no worse than the optimal solutions of the former in terms of system objectives, because the latter has a larger solution space which strictly contains the solution space of the former.

\subsubsection{Online Optimization over Time}

Most of this thesis only handles the "offline" case. The inputs such as each user's storage cost and traffic cost, each user's numbers of read and write operations are static, given, and fixed. The decisions of where to place data are also made for only one time. Even in Chapter 3 where we consider a series of time periods and decisions need to be made for multiple times, we treat each time period as 
a separate and independent data placement problem and aim to optimize the monetary expense in each period for its own sake. For real-world services, however, a more intriguing model may aim to optimize the total monetary expense or various system objectives over time, which desires an "online" algorithm. For instance, an ideal online algorithm may run at every time period based on the information about the current time period and also the pervious time periods, while ensuring that the total expense over time is within a bounded gap to the offline optimum, i.e., the optimal total expense over time assuming the information about every period is known in prior. Techniques including Lyapunov optimization $[36,59,92,97,100,101]$ would be a candidate framework to design online algorithms with guaranteed bounds; we may also need to modify our models, making it solvable by such techniques but still capture the real-world scenarios. One may also investigate and discuss, assuming a certain amount of future information is known in prior, how much closer the online optimum can be to the offline optimum compared with the case where no future information is known at all.

\subsubsection{A Game Theoretic Perspective}

No matter placing OSN data across clouds or inside a cloud, this thesis investigates where to place the data of a single service; however, in reality, a cloud as a common infrastructure is often shared by multiple services which may belong to different service providers. Given a group of geo-distributed clouds, each service provider makes decisions about where to place the data of its service in order to selfishly pursue the optimization of its own system performance or objectives. In such a scenario, the performance of one service can be affected by the other service if both services share a common cloud or a common server. Let's consider the following example of access latency. The access latency of a service perceived by a user depends on the out-of-cloud latency, which is basically the network latency between a user and the cloud, and the in-cloud latency, which is the latency inside the cloud including the time of waiting and processing. While the former part is our focus in this thesis, i.e., selecting a cloud by considering its proximities to users with the assumption that proximity is a good approximation to network latency, the latter part has not been addressed in this thesis. If one service occupies a server exclusively, it has all the server's CPU time; but if there are two services isolated but co-located at a common server, perhaps each service only acquires half of the server's CPU time on average. Consequently, the latency of processing a request in the sharing case is twice as much as that in the exclusive case. This is a typical multi-party scenario that can be captured by game theory $[18,38,42,67,69,78]$ : a service is a player; a placement of users' data of a service is a strategy of a player, and all possible placements compose a 
player's strategy set; the access latency of a service, which depends on all players' strategies, is the payoff of a player. Then we have a static, pure-strategy game. Issues that may be interesting include whether this game has a pure-strategy Nash equilibrium where every service cannot provide shorter access latency to its users by unilaterally changing its data placement, and how close this equilibrium, if exists, is to the social optimal placement where the sum of the latencies of all services is globally optimal. The challenges for investigating such issues lie in that the strategy set of each player, though finite, is large and the payoff function is complicated. 



\section{Bibliography}

[1] Amazon EC2 Pricing. http://aws .amazon.com/ec2/pricing.

[2] Case Studies. http://aws.amazon.com/solutions/case-studies.

[3] Data Center Map. http://www.datacentermap.com/cloud.html.

[4] Facebook Newsroom. http://newsroom.fb.com.

[5] How to Use Facebook for Business Marketing. http://www.facebook. com/business/overview.

[6] Pricing Overview. http://www.windowsazure.com/pricing.

[7] Twitter/Gizzard. http://github.com/twitter/gizzard.

[8] U.S. Board on Geographic Names (BGN). http://geonames usgs.gov/.

[9] U.S. Energy Information Administration (EIA). http://www.eia.gov/.

[10] State of the Twittersphere. HubSpot, Inc., Jan. 2010.

[11] Cisco Data Center Infrastructure 2.5 Design Guide. Cisco Systems, Inc., Nov. 2011.

[12] A. Abou-Rjeili and G. Karypis. Multilevel algorithms for partitioning power-law graphs. In IPDPS, 2006.

[13] S. Agarwal, J. Dunagan, N. Jain, S. Saroiu, A. Wolman, and H. Bhogan. Volley: Automated data placement for geo-distributed cloud services. In NSDI, 2010.

[14] M. Al-Fares, A. Loukissas, and A. Vahdat. A scalable, commodity data center network architecture. In SIGCOMM, 2008.

[15] M. Al-Fares, S. Radhakrishnan, B. Raghavan, N. Huang, and A. Vahdat. Hedera: Dynamic flow scheduling for data center networks. In NSDI, 2010.

[16] M. Alicherry and T.V. Lakshman. Network aware resource allocation in distributed clouds. In INFOCOM, 2012. 
[17] M. Alizadeh, A. Greenberg, D.A. Maltz, J. Padhye, P. Patel, B. Prabhakar, S. Sengupta, and M. Sridharan. Data center tcp (dctcp). In SIGCOMM, 2011.

[18] D. Ardagna, B. Panicucci, and M. Passacantando. A game theoretic formulation of the service provisioning problem in cloud systems. In $W W W$, 2011.

[19] L. Backstrom, D. Huttenlocher, J. Kleinberg, and X. Lan. Group formation in large social networks: membership, growth, and evolution. In SIGKDD, 2006.

[20] J. Baker, C. Bond, J.C. Corbett, JJ Furman, A. Khorlin, J. Larson, J.M. Léon, Y. Li, A. Lloyd, and V. Yushprakh. Megastore: Providing scalable, highly available storage for interactive services. In CIDR, 2011.

[21] A.L. Barabasi. The origin of bursts and heavy tails in human dynamics. Nature, 435(7039):207-211, 2005.

[22] F. Benevenuto, T. Rodrigues, M. Cha, and V. Almeida. Characterizing user behavior in online social networks. In $I M C, 2009$.

[23] T. Benson, A. Akella, and D.A. Maltz. Network traffic characteristics of data centers in the wild. In $I M C, 2010$.

[24] K.D. Bowers, A. Juels, and A. Oprea. Hail: a high-availability and integrity layer for cloud storage. In $C C S, 2009$.

[25] Y. Boykov and V. Kolmogorov. An experimental comparison of mincut/max-flow algorithms for energy minimization in vision. IEEE Transactions on Pattern Analysis and Machine Intelligence, 26(9):1124-1137, 2004 .

[26] Y. Boykov, O. Veksler, and R. Zabih. Fast approximate energy minimization via graph cuts. IEEE Transactions on Pattern Analysis and Machine Intelligence, 23(11):1222-1239, 2001.

[27] B. Carrasco, Y. Lu, and J. Trindade. Partitioning social networks for time-dependent queries. In EuroSys SNS, 2011.

[28] E. Cecchet, R. Singh, U. Sharma, and P. Shenoy. Dolly: virtualizationdriven database provisioning for the cloud. In VEE, 2011.

[29] F. Chen, K. Guo, J. Lin, and T. La Porta. Intra-cloud lightning: Building cdns in the cloud. In INFOCOM, 2012. 
[30] H. Chen, H. Jin, N. Jin, and T. Gu. Minimizing inter-server communications by exploiting self-similarity in online social networks. In ICNP, 2012.

[31] X. Cheng and J. Liu. Load-balanced migration of social media to content clouds. In NOSSDAV, 2011.

[32] M. Chowdhury, S. Kandula, and I. Stoica. Leveraging endpoint flexibility in data-intensive clusters. In SIGCOMM, 2013.

[33] H. Chun, H. Kwak, Y.H. Eom, Y.Y. Ahn, S. Moon, and H. Jeong. Comparison of online social relations in volume vs interaction: a case study of cyworld. In $I M C, 2008$.

[34] A. Ciuffoletti. Monitoring a virtual network infrastructure: an iaas perspective. ACM SIGCOMM Computer Communication Review, 40(5):4752,2010 .

[35] C. Curino, E. Jones, Y. Zhang, and S. Madden. Schism: a workload-driven approach to database replication and partitioning. In $V L D B, 2010$.

[36] W. Deng, F. Liu, H. Jin, C. Wu, and X. Liu. Multigreen: Cost-minimizing multi-source datacenter power supply with online control. In $A C M e$ Energy, 2013.

[37] Q. Duong, S. Goel, J. Hofman, and S. Vassilvitskii. Sharding social networks. In WSDM, 2013.

[38] Y. Feng, B. Li, and B. Li. Bargaining towards maximized resource utilization in video streaming datacenters. In INFOCOM, 2012.

[39] L.R. Ford and D.R. Fulkerson. Maximal flow through a network. Canadian Journal of Mathematics, 8(3):399-404, 1956.

[40] P.X. Gao, A.R. Curtis, B. Wong, and S. Keshav. It's not easy being green. In SIGCOMM, 2012.

[41] C. Guo, G. Lu, D. Li, H. Wu, X. Zhang, Y. Shi, C. Tian, Y. Zhang, and S. Lu. Bcube: a high performance, server-centric network architecture for modular data centers. In SIGCOMM, 2009.

[42] J. Guo, F. Liu, D. Zeng, J. Lui, and H. Jin. A cooperative game based allocation for sharing data center networks. In INFOCOM, 2013.

[43] Z. Guo, Z. Zhang, and Y. Yang. Exploring server redundancy in nonblocking multicast data center networks. In INFOCOM, 2012. 
[44] M. Hajjat, X. Sun, Y.-W. E. Sung, D. Maltz, S. Rao, K. Sripanidkulchai, and M. Tawarmalani. Cloudward bound: planning for beneficial migration of enterprise applications to the cloud. In SIGCOMM, 2010.

[45] I. Hoque and I. Gupta. Disk layout techniques for online social network data. IEEE Internet Computing, 16(3):24-36, 2012.

[46] H. Hu and X. Wang. Evolution of a large online social network. Physics Letters A, 373(12-13):1105-1110, 2009.

[47] V. Jalaparti, P. Bodik, S. Kandula, I. Menache, M. Rybalkin, and C. Yan. Speeding up distributed request-response workflows. In SIGCOMM, 2013.

[48] J. Jiang, C. Wilson, X. Wang, P. Huang, W. Sha, Y. Dai, and B.Y. Zhao. Understanding latent interactions in online social networks. In $I M C, 2010$.

[49] A. Juels and A. Oprea. New approaches to security and availability for cloud data. Communications of the ACM, 56(2):64-73, 2013.

[50] S. Kadambi, J. Chen, B. Cooper, D. Lomax, R. Ramakrishnan, A. Silberstein, E. Tam, and H. Garcia-Molina. Where in the world is my data? In $V L D B, 2011$.

[51] A. Kaltenbrunner, S. Scellato, Y. Volkovich, D. Laniado, D. Currie, E.J. Jutemar, and C. Mascolo. Far from the eyes, close on the web: impact of geographic distance on online social interactions. In SIGCOMM WOSN, 2012.

[52] G. Karypis. METIS: A Software Package for Partitioning Unstructured Graphs, Partitioning Meshes, and Computing Fill-Reducing Orderings of Sparse Matrices. 2013.

[53] G. Karypis and V. Kumar. A fast and high quality multilevel scheme for partitioning irregular graphs. SIAM Journal on Scientific Computing, 20(1):359-392, 1999.

[54] A. Khanafer, M. Kodialam, and K.P.N. Puttaswamy. The constrained skirental problem and its application to online cloud cost optimization. In INFOCOM, 2013.

[55] S. Kirkpatrick, C.D. Gelatt, and M.P. Vecchi. Optimization by simmulated annealing. Science, 220(4598):671-680, 1983.

[56] V. Kolmogorov and R. Zabin. What energy functions can be minimized via graph cuts? IEEE Transactions on Pattern Analysis and Machine Intelligence, 26(2):147-159, 2004. 
[57] A. Lakshman and P. Malik. Cassandra: a decentralized structured storage system. ACM SIGOPS Operating Systems Review, 44(2):35-40, 2010.

[58] K. Le, O. Bilgir, R. Bianchini, M. Martonosi, and T.D. Nguyen. Managing the cost, energy consumption, and carbon footprint of internet services. In SIGMETRICS, 2010.

[59] S. Li, Y. Zhou, L. Jiao, X. Yan, X. Wang, and M.R. Lyu. Delay-aware cost optimization for dynamic resource provisioning in hybrid clouds. In ICWS, 2014.

[60] G. Liu, H. Shen, and H. Chandler. Selective data replication for online social networks with distributed datacenters. In ICNP, 2013.

[61] Z. Liu, M. Lin, A. Wierman, S.H. Low, and L.L.H. Andrew. Greening geographical load balancing. In SIGMETRICS, 2011.

[62] X. Meng, V. Pappas, and L. Zhang. Improving the scalability of data center networks with traffic-aware virtual machine placement. In INFOCOM, 2010 .

[63] A. Mislove. Online Social Networks: Measurement, Analysis, and Applications to Distributed Information Systems. PhD thesis, Rice University, 2009 .

[64] A. Mislove, M. Marcon, K.P. Gummadi, P. Druschel, and B. Bhattacharjee. Measurement and analysis of online social networks. In $I M C, 2007$.

[65] M. Mondal, B. Viswanath, A. Clement, P. Druschel, K.P. Gummadi, A. Mislove, and A. Post. Defending against large-scale crawls in online social networks. In CoNEXT, 2012.

[66] J. Mudigonda, P. Yalagandula, J. Mogul, B. Stiekes, and Y. Pouffary. Netlord: a scalable multi-tenant network architecture for virtualized datacenters. In SIGCOMM, 2011.

[67] A. Nahir, A. Orda, and D. Raz. Workload factoring with the cloud: A game-theoretic perspective. In INFOCOM, 2012.

[68] G.L. Nemhauser and L.A. Wolsey. Integer and combinatorial optimization. Wiley New York, 1988.

[69] D. Niu, C. Feng, and B. Li. A theory of cloud bandwidth pricing for video-on-demand providers. In INFOCOM, 2012.

[70] F. Pellegrini and J. Roman. Scotch: A software package for static mapping by dual recursive bipartitioning of process and architecture graphs. In HPCN Europe, 1996. 
[71] L. Popa, G. Kumar, M. Chowdhury, A. Krishnamurthy, S. Ratnasamy, and I. Stoica. Faircloud: sharing the network in cloud computing. In SIGCOMM, 2012.

[72] J.M. Pujol, V. Erramilli, G. Siganos, X. Yang, N. Laoutaris, P. Chhabra, and P. Rodriguez. The little engine(s) that could: Scaling online social networks. In SIGCOMM, 2010.

[73] J.M. Pujol, V. Erramilli, G. Siganos, X. Yang, N. Laoutaris, P. Chhabra, and P. Rodriguez. The little engine(s) that could: Scaling online social networks. IEEE/ACM Transactions on Networking, 20(4):1162-1175, 2012.

[74] A. Qureshi, R. Weber, H. Balakrishnan, J. Guttag, and B. Maggs. Cutting the electric bill for internet-scale systems. In SIGCOMM, 2009.

[75] C. Raiciu, S. Barre, C. Pluntke, A. Greenhalgh, D. Wischik, and M. Handley. Improving datacenter performance and robustness with multipath tcp. In SIGCOMM, 2011.

[76] L. Rao, X. Liu, L. Xie, and W. Liu. Minimizing electricity cost: Optimization of distributed internet data centers in a multi-electricity-market environment. In INFOCOM, 2010.

[77] Y. Rochman, H. Levy, and E. Brosh. Resource placement and assignment in distributed network topologies. In INFOCOM, 2013.

[78] H. Roh, C. Jung, W. Lee, and D.-Z. Du. Resource pricing game in geodistributed clouds. In INFOCOM, 2013.

[79] K. Schloegel, G. Karypis, and V. Kumar. Wavefront diffusion and lmsr: Algorithms for dynamic repartitioning of adaptive meshes. IEEE Transactions on Parallel and Distributed Systems, 12(5):451-466, 2001.

[80] Y. Sovran, R. Power, M.K. Aguilera, and J. Li. Transactional storage for geo-replicated systems. In SOSP, 2011.

[81] D.A. Tran, K. Nguyen, and C. Pham. S-clone: Socially-aware data replication for social networks. Computer Networks, 56(7):2001-2013, 2012.

[82] N. Tran, M.K. Aguilera, and M. Balakrishnan. Online migration for geodistributed storage systems. In USENIX ATC, 2011.

[83] L.M. Vaquero, L. Rodero-Merino, and R. Buyya. Dynamically scaling applications in the cloud. ACM SIGCOMM Computer Communication Review, 41(1):45-52, 2011. 
[84] A. Vázquez, J.G. Oliveira, Z. Dezsö, K.I. Goh, I. Kondor, and A.L. Barabási. Modeling bursts and heavy tails in human dynamics. Physical Review E, 73(3):036127, 2006.

[85] B. Viswanath, A. Mislove, M. Cha, and K.P. Gummadi. On the evolution of user interaction in facebook. In SIGCOMM WOSN, 2009.

[86] Z. Wang, L. Sun, X. Chen, W. Zhu, J. Liu, M. Chen, and S. Yang. Propagation-based social-aware replication for social video contents. In ACM Multimedia, 2012.

[87] X. Wen, K. Chen, Y. Chen, Y. Liu, Y. Xia, and C. Hu. Virtualknotter: Online virtual machine shuffling for congestion resolving in virtualized datacenter. In ICDCS, 2012.

[88] P. Wendell, J.W. Jiang, M.J. Freedman, and J. Rexford. Donar: decentralized server selection for cloud services. In SIGCOMM, 2010.

[89] C. Wilson, H. Ballani, T. Karagiannis, and A. Rowtron. Better never than late: Meeting deadlines in datacenter networks. In SIGCOMM, 2011.

[90] C. Wilson, B. Boe, A. Sala, K.P.N. Puttaswamy, and B.Y. Zhao. User interactions in social networks and their implications. In EuroSys, 2009.

[91] M.P. Wittie, V. Pejovic, L. Deek, K.C. Almeroth, and B.Y. Zhao. Exploiting locality of interest in online social networks. In CoNEXT, 2010.

[92] Y. Wu, C. Wu, B. Li, L. Zhang, Z. Li, and F.C.M. Lau. Scaling social media applications into geo-distributed clouds. In INFOCOM, 2012.

[93] Z. Wu, M. Butkiewicz, D. Perkins, E. Katz-Bassett, and H.V. Madhyastha. Spanstore: Cost-effective geo-replicated storage spanning multiple cloud services. In $S O S P, 2013$.

[94] Z. Wu and H.V. Madhyastha. Understanding the latency benefits of multicloud webservice deployments. ACM SIGCOMM Computer Communication Review, 43(1):13-20, 2013.

[95] H. Xu and B. Li. Joint request mapping and response routing for geodistributed cloud services. In INFOCOM, 2013.

[96] Y. Yang, Q. Chen, and W. Liu. The structural evolution of an online discussion network. Physica A: Statistical Mechanics and its Applications, 389(24):5871-5877, 2010.

[97] Y. Yao, L. Huang, A. Sharma, L. Golubchik, and M. Neely. Data centers power reduction: A two time scale approach for delay tolerant workloads. In INFOCOM, 2012. 
[98] L. Zhang, C. Wu, Z. Li, C. Guo, M. Chen, and F.C.M. Lau. Moving big data to the cloud: An online cost-minimizing approach. IEEE Journal on Selected Areas in Communications, 31(12):2710-2721, 2013.

[99] Z. Zhang, M. Zhang, A. Greenberg, Y.C. Hu, R. Mahajan, and B. Christian. Optimizing cost and performance in online service provider networks. In NSDI, 2010.

[100] J. Zhao, H. Li, C. Wu, Z. Li, Z. Zhang, and F.C.M. Lau. Dynamic pricing and profit maximization for the cloud with geo-distributed data centers. In INFOCOM, 2014.

[101] Z. Zhou, F. Liu, H. Jin, B. Li, B. Li, and H. Jiang. On arbitrating the power-performance tradeoff in saas clouds. In INFOCOM, 2013.

[102] Z. Zhou, F. Liu, B. Li, B. Li, H. Jin, R. Zou, and Z. Liu. Fuel cell generation in geo-distributed cloud services: A quantitative study. In ICDCS, 2014. 